\title{
Affecting Factors in Information Security Policy Compliance: Combine Organisational Factors and User Habits
}

\author{
Angraini $^{1,4}$, Rose Alinda Alias ${ }^{2}$ and Okfalisa ${ }^{3}$ \\ 1School of Computing, Faculty Engineering, University Technology Malaysia, 81310 Johor, \\ Malaysia \\ ${ }_{2}$ Department of Information System, Azman Hashim International Business School, University \\ Technology Malaysia,81310 Johor Malaysia \\ 3Department of Informatics Engineering, Faculty Science and Technology, Universitas Islam \\ Negeri Sultan Syarif Kasim, Pekanbaru, Riau \\ 4Department of Information System, Faculty Science and Technology, Universitas Islam \\ Negeri Sultan Syarif Kasim, Pekanbaru, Riau \\ Angraini@uin-suska.ac.id
}

\begin{abstract}
Information security policy compliance is one concern of organisations to improve information security, including universities. Previous research has shown that factors that influence user compliance can come from user behaviour and from within the organisation. This study aims to explore the factors of organisation and user habits that affect user compliance with information security policies. The research model proposed used organisational commitment, organisational culture, reward, and habit variables - this research conducted with a case study of public universities in Indonesia by using an online survey. The results indicated that the factors derived from organisational commitment established a positive influence on the user's respectful behaviour. The insignificant organisational culture and reward affected information security policies. User habits also influenced user behaviour in maintaining information security.
\end{abstract}

Keywords: compliance, information security policy, organisation commitment, organisation culture, habit.

\section{Introduction}

There has been a growth in research to devote more attention to information security due to a significant increase in threats and attacks on information security. Cybersecurity Ventures estimates that a business will fall victim to ransomware attacks every 14 seconds in 2019 and every 11 seconds in 2021 [1]. universities are one of targeting information security attacks because they have confidential data.(Symantec, 2 017, 2018). An increase in the number of security violations experienced in recent years by higher education than the importance of confidentiality, integrity, and availability of information at the university (Bongiovanni 2019). 
Information Security protection conducted technically and strategically by using security policies as a guide for their employees to protect user information. The organisation considers system users are considered the most unprotected part of the information security system [2]. Almost $60 \%$ of IT managers believe employee negligence to establish an organisation lose millions of dollars as a severe threat to information security [3]. The organisation is aware of the much information security threat that can come from inside or outside the organisation. Therefore, it is essential for organisations always to ensure their information security policies are applied continuously. However, employees are often even unprepared to comply with these procedures and guidelines. Whereas, information system security policies made to secure the company IS assets and prevent misuse of the destruction of its information system $[4,5]$. Hence, it is necessary to recognise the factors that influence users to comply with security policies which influence users to adhere to information security policies can originate from organisational or human behaviour. Several researchers have examined to determine the factors that influence users to comply with information security policies. Human and organisational factors represent factors that influence compliance with information security policies [6]

Empirical Data from D'Arcy (2014) show that a culture of security can cause employees to comply with information security policies in the workplace [7]. Whereas according to Chang (2012), organisational commitment is another factor related to the behaviour of organisational citizens, employees believe their security-conscious behaviour might cause a beneficial effect on the organisation's overall information security [8]. Organisations also need rewarding them for receiving improvements in security compliance if they want to improve compliance with information security policies [9]. This similar problem also encountered by Sommestad (2017) found a tendency to be obedient due to the habits from previous behaviour [10]. Recent Researchers agreed on the role of organisation, and the end-user is essential for information security, and several studies are examining the relationship between the role of organisations and individuals. However, limited studies investigated the relationship between organisational variables and user behaviour research on specific types of organisations such as a university. This study is to explore the factors that influence user compliance from the organisations' perspective and user habits.

The remaining part of the paper as follows: introduction, literature review, research methodology, research finding, discussion, and conclusion. This paper begins by describing a research background in the introduction section. It will then go on to section review from previous studies. After that, continue to research methodology to explain how this study conduct. The research finding explains in the following section and continues to discuss the research finding. The preceding part will discuss the limitation and conclusions 


\section{Literature Review}

It is essential to possess a consciousness about the current issues highlighted in the literature on compliance with information security policies. Information security policies contain rules employed to create organisational IT security rules, specific problems, and system policies to address individual systems [11]. Policy documents must be obliged with precise controls and procedures for employees to implement. Otherwise, policies must be specific and detailed; hence, users can follow the guidelines [12]. Problems can arise in utilising information on security policies that become sourced from present organisations and individuals. The weakest information security chains are individuals. Therefore, it is necessary to understand how to return people in the organisation into partners for increased information security.

\subsection{Organisation commitment}

Measurement of organisational commitment was first introduced by Mowday (1979) to find out how employee commitment works at the organisation [13]. However, not excessively significant organisational commitment affects user behaviour. This pattern shows the impact of employee commitment on the organisation may depend on the particular behaviour that is the employee's commitment and the effect of the behaviour on organisational results [14].

The effect of an individual's prior commitment to an institution on their reaction to the perceived fairness of decisions given by the institution examined in two different field settings [15]. Information technology user behaviour in organisations can be related to the commitment to the organisation. In information security, these employees believe their security-conscious behaviour affects the overall information security of the organisation. Thus, the level of organisational commitment influences the intention to follow security policies [8]. Organisational commitment focuses on staff commitment to the organisation. Organisational commitment is the most developed of all work commitment constructs [16].

\subsection{Organisation culture}

The organisation seeks to encourage employees to comply with information security policies when losses due to information breaches become serious. Therefore, the attention demanded such behaviour and social behaviour as an effort to constitute a substantial theoretical foundation related to security behaviour [17]. Organisations should develop policies appropriate to the culture of the organisation. Because according to Alshare (2018), organisational culture is a significant predictor for determining crime against information security policies [18]. Likewise, research conducted by Arage (2015) stated that national culture affects compliance with information security [19]. Another factor in organisations is user involvement and leadership, this potential is seen by Amankwa (2018) in his research on building a culture of compliance with information security policies in organisations using theories of organisational behaviour and organisational culture [20]. An ideal organisation 
Culture prompts conscious and knowledgeable users who need to think about policies implemented by management. Organisations have reliable information security that enhances mutual trust and integration through securing their information [21].

\subsection{Reward}

The regulations developed must be forced on users to ensure users always obey, or users get a direct impact if they comply with policies such as getting gifts or getting penalties. The reward factor mentioned by the interviewee will increase employee compliance with information security policies. The reward will support the achievement of performance goals and improve security compliance [9], although some researches show reward does not have a significant impact on intentions to comply with information security policies $[22,23]$. This study attempted exploring the importance of rewards with compliance with information security policies.

\subsection{Habit}

Several researchers have examined, a habit has become one time that influences users to comply with Pahnila's information security policy [22, 24, 25]. Sommestad (2017) conducted an empirical study to develop a theory of planned behaviour used explicitly for information security compliance by adding regret and anticipated habits. The results of his research found that habits did not significantly influence behavioural intentions to comply with information security policies [26]. Users will adhere to ISPs out of habit, consider them essential, and not remain a barrier to their work [27]. If people have developed good behaviour habits of information security policy compliance, compliance with their information security policies will be automatic [28]. Therefore, further research is required to use this variable with different research objects.

Based on the literature review related to user behaviour compliance with information security policies, we proposed a research model described in Fig.1.

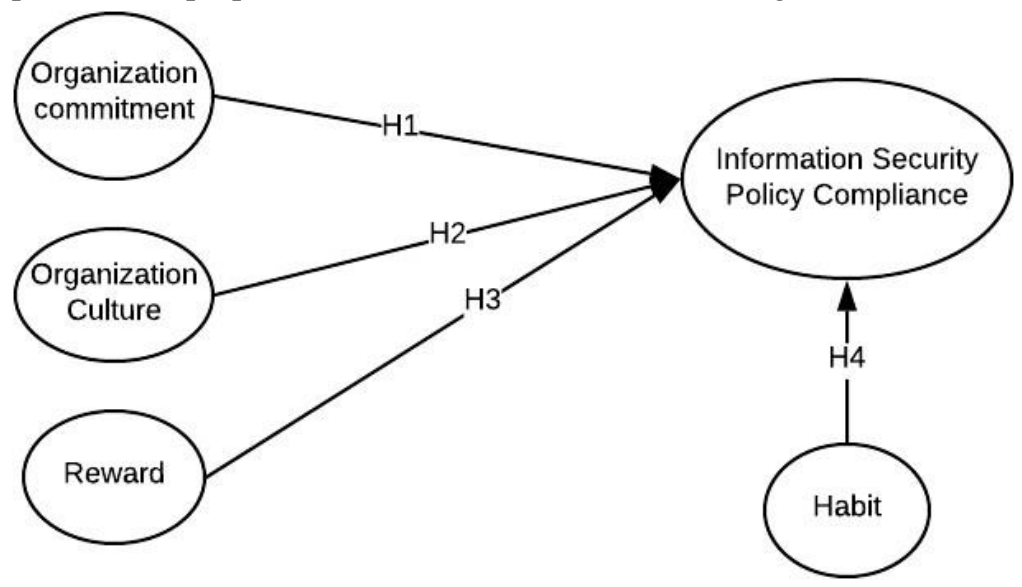

Fig. 1. Research model 
This research model consisted of four variables: organisational commitment, organisation culture, reward, and habit.

The hypothesis for this study was as follows:

Hypothesis 1. organisation commitment influenced a user to comply with information security policies.

Hypothesis 2. organisation culture influenced to comply with information security policies

Hypothesis 3. reward affected users to comply with information security policy.

Hypothesis 4. habit influenced a user to comply with information security policies

\section{Research Methodology}

Case study for this research conducted in public universities in Indonesia. The method attempts solving the problem by conducting an online survey. Research progress describes at Fig.2 on below.

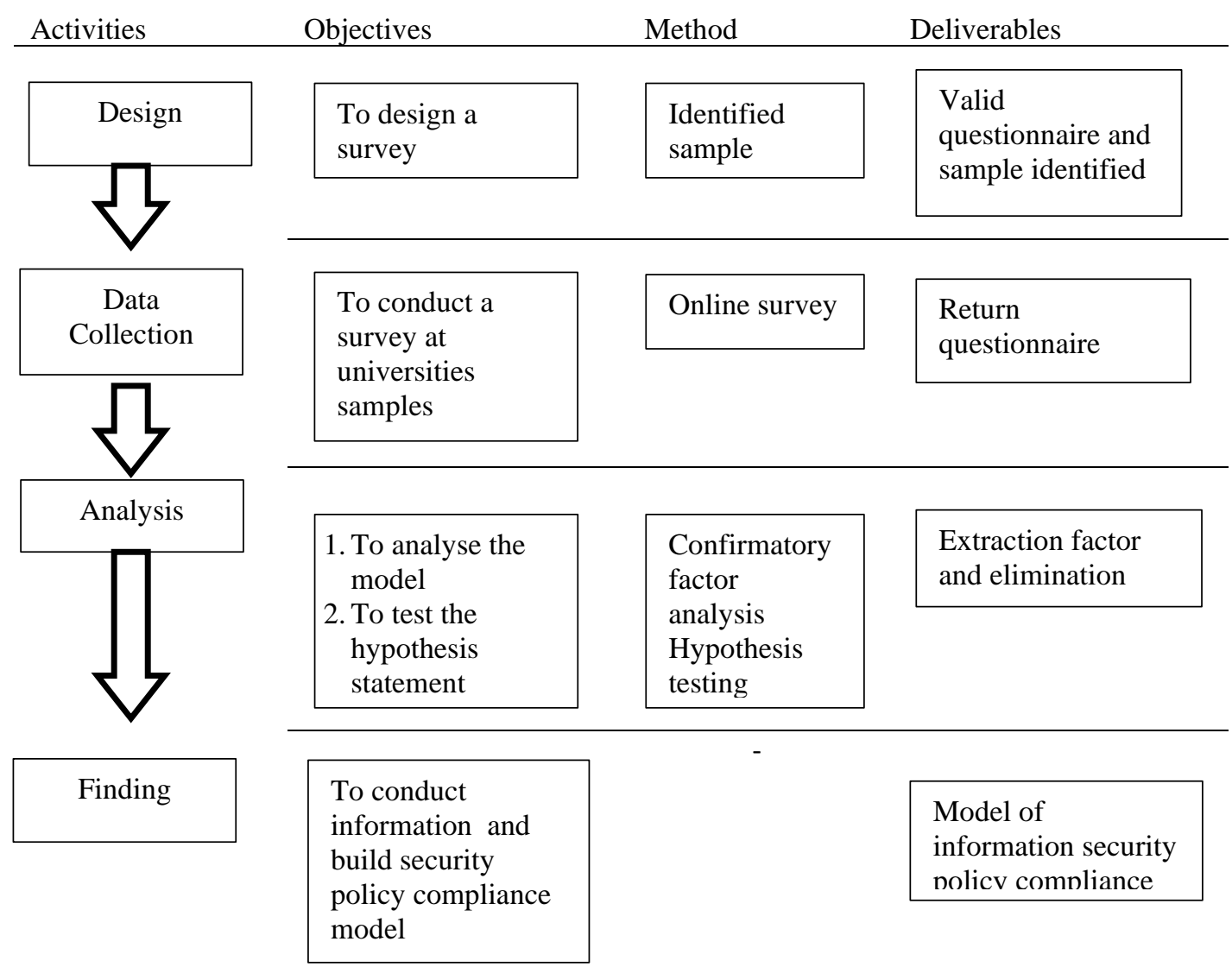

Fig. 2. Research progress 
The questionnaire adapted from previous researchers: organizations' commitment [29], organization's culture [30, 31], rewards [22, 23, 32, 33] and habits [10, 22]. Invitation for the survey sends by email to university as and the questionnaire created with a google form. Online survey research can reach participants widespread as long as they have an internet connection and require anonymity [34]

The online survey conducted for two months obtained by 430 participants. Continue with data screening and encountered 412 respondents to analyse. Demography of respondents, including male $(65 \%)$, women (35\%), with age range $25-29$ years $(10 \%)$, $30-35$ years $(18 \%), 36-40$ years $(27 \%), 41-45$ Years $(20 \%)$, above 45 years old $(25 \%)$. $80 \%$ of respondents hold Masters and $\mathrm{PhD} .20 \%$ of respondents have work experience under two years, 3-5 years (6\%), 6-10 years 98 (24\%), and above ten years (65\%). 9\% of respondents occupy prominent positions at the university level, faculty level $15 \%$, department level 46\%, and lectures 30\%. This research used Partial Least Structure (PLS) to analyse the data because this study was exploratory and used reflective variables [35].

\section{$4 \quad$ Research Finding}

Convergent validity used to measure model with the reflective indicator assessed based on the correlation between the component score item estimated with software PLS. Individual Reflective size was said to be high if it correlated with more than 0.70 with a measured construct. However, the early research phase of the development scale of the measurement of the loading factor in range 0.5 to 0.6 was considered adequate. In this study, it used to limit the loading factor of 0.50 . Furthermore, discriminant validity carried out to ensure that each indicator of each latent variable differs from the other variables. The model maintained good discriminant validity if each loading value of each indicator of a latent variable possessed a more considerable loading value than other latent variables. Table 1 describes the result of convergent validity 
Table 1. Convergent validity

\begin{tabular}{|c|c|c|c|c|c|}
\hline Construct & Item code & $\begin{array}{l}\text { Outer } \\
\text { loading }\end{array}$ & $\begin{array}{l}\text { Cronbach } \\
\text { alpha }\end{array}$ & $\begin{array}{l}\text { Composite } \\
\text { reliability }\end{array}$ & $\begin{array}{l}\text { The average variance } \\
\text { extracted (AVE) }\end{array}$ \\
\hline \multirow{8}{*}{$\begin{array}{l}\text { Organisation } \\
\text { commitment (OC) }\end{array}$} & OC 1 & 0.909 & \multirow[t]{8}{*}{0.949} & \multirow[t]{8}{*}{0.958} & \multirow[t]{8}{*}{0.739} \\
\hline & OC 2 & 0.885 & & & \\
\hline & OC 3 & 0.831 & & & \\
\hline & OC 4 & 0.816 & & & \\
\hline & OC 5 & 0.887 & & & \\
\hline & OC 6 & 0.885 & & & \\
\hline & OC 7 & 0.83 & & & \\
\hline & OC 8 & 0.83 & & & \\
\hline \multirow{6}{*}{$\begin{array}{l}\text { Organisation Culture } \\
\text { (OL) }\end{array}$} & OL 1 & 0.972 & \multirow[t]{6}{*}{0.968} & \multirow[t]{6}{*}{0.974} & \multirow[t]{6}{*}{0.861} \\
\hline & OL 2 & 0.969 & & & \\
\hline & OL 3 & 0.923 & & & \\
\hline & OL 4 & 0.922 & & & \\
\hline & OL 5 & 0.926 & & & \\
\hline & OL 6 & 0.85 & & & \\
\hline \multirow[t]{6}{*}{ Reward (RW) } & RW 1 & 0.822 & \multirow[t]{6}{*}{0.88} & \multirow[t]{6}{*}{0.898} & \multirow[t]{6}{*}{0.596} \\
\hline & RW 2 & 0.839 & & & \\
\hline & RW 3 & 0.736 & & & \\
\hline & RW 4 & 0.807 & & & \\
\hline & RW 5 & 0.759 & & & \\
\hline & RW 6 & 0.654 & & & \\
\hline \multirow[t]{7}{*}{ Habit (HA) } & HA 1 & 0.975 & \multirow[t]{7}{*}{0.984} & \multirow[t]{7}{*}{0.986} & \multirow[t]{7}{*}{0.911} \\
\hline & HA 2 & 0.966 & & & \\
\hline & HA 3 & 0.948 & & & \\
\hline & HA 4 & 0.947 & & & \\
\hline & HA 5 & 0.946 & & & \\
\hline & HA 6 & 0.947 & & & \\
\hline & HA 7 & 0.952 & & & \\
\hline \multirow{3}{*}{$\begin{array}{l}\text { Information security } \\
\text { policy compliance (ISPC) }\end{array}$} & ISP1 & 0.888 & \multirow[t]{3}{*}{0.848} & \multirow[t]{3}{*}{0.899} & \multirow[t]{3}{*}{0.692} \\
\hline & ISP2 & 0.812 & & & \\
\hline & $\begin{array}{l}\text { ISP3 } \\
\text { ISP4 }\end{array}$ & $\begin{array}{l}0.714 \\
0.9 \\
\end{array}$ & & & \\
\hline
\end{tabular}


Table 1 explains that all outer loading values are above 0.5 so that the measurement model requirements acceptable. The loading factor value for each indicator of each latent variable did not have the most significant loading factor value compared to the loading value if it was associated with other potential variables. This value means that each latent variable had a good discriminant validity where some latent variables did not have gauges positively correlated with other constructs. Validity and reliability criteria can found from the reliability value of a construct and the value of the Average Variance Extracted (AVE) of each construct. The construct was said to have high reliability if the value was 0.70 and AVE was above 0.50 . All constructs had composite reliability values above 0.70 and Cronbach's alpha above 0.60 . It concluded that the construct had excellent reliability.

In PLS statistical testing, every hypothesised relationship carried out using simulations. In this case, the bootstrap method performed on the sample. Bootstrap testing also intended to minimise the problem of research data abnormalities. The estimated significance parameters provided beneficial information regarding the relationship between the research variables. Bootstrapping test results from PLS analysis could be seen in the outcome for inner weight output and testing the hypothesis of direct influence between variables based on bootstrapping presented in table 2 .

Table 2. Summary of hypotheses testing

\begin{tabular}{llllllll}
\hline & $\begin{array}{l}\text { Std. } \\
\text { Beta }\end{array}$ & t-value & p values & Bias & \multicolumn{2}{c}{ Confidence interval } & Decision \\
& & & & $2.50 \%$ & $97.50 \%$ & \\
\hline organization commitment -> ISP & 0.042 & 14.297 & 0 & -0.001 & 0.511 & 0.671 & Supported \\
organization culture -> ISP & 0.04 & 0.902 & 0.367 & -0.001 & -0.072 & 0.1 & rejected \\
reward -> ISP & 0.058 & 0.559 & 0.576 & 0.003 & -0.165 & 0.086 & rejected \\
Habit -> ISP & 0.043 & 3.751 & 0 & -0.002 & 0.084 & 0.247 & Supported \\
\hline
\end{tabular}

There were four hypotheses in this study; H4, habit had a significant influence on information security policy compliance. The t-value of 3.751 was higher than the value of t-table and p-value $0.000<0.05$. The results showed that this hypothesis was acceptable and showed important habits in information security policy compliance. Second, H1 also had a very significant influence on organisational commitment. Tvalue 14,297 showed this variable to be the most considerable compared to other variables. However, the $\mathrm{H} 2$ and $\mathrm{H} 3 \mathrm{t}$-value and p-value indicated no contribution and significant towards user compliance with information security policies. As a result, the organisational culture and reward variables concluded to have no relationship and influence on information security policies. 


\section{Discussion}

The results showed that $\mathrm{H} 1$ and $\mathrm{H} 4$ were supported, while for $\mathrm{H} 2$ and $\mathrm{H} 3$, the hypothesis rejected. This finding showed that user habits in maintaining information security affected compliance with information security policies. This study stated that user habits played a role in their behaviour in complying with information security policies. Similar to results found by Moody (2018), habit is a predictor that can be a predictor of user behaviour in complying with information security policies and affect intentions of precautionary action [22]. The habit of protecting information security was past behaviour, and behaviour might carry out before the information security policy implemented; therefore, it became a concern in use it to measuring compliance with information security policies. As stated by Sommestad (2017), habits can increase compliance with policies, but not as much as other behaviours [10].

Furthermore, the role of the organisation's commitment was enormous in increasing compliance with information security policies. Users would better maintain information security behaviour when they realised the organisation had responsibility for information security. Organisational commitment needed to apply to improve user security behaviour. Regulations had a direct effect on information security compliance showed organisational commitment, and then it was essential to have institutional pressure for effective information security compliance [36]. This study showed that organisational commitment variables were significant in improving user compliance behaviour towards information security policies.

The results that surprised researchers were the absence of the role of reward and organisational culture for compliance with information security policies. This study conducted with a case study at the university, so it was possible to find different results. Contrary to the research of Bulgurcu (2010), which state the rewards will impact user compliance with the policy [32]. Although previous research by Pahnila (2007), rewards do not have a significant effect on actual compliance with IS security policies [24]. Compensation used for the achievement of an objective will negatively impact security compliance [9]. Therefore, a reward given by organisations hoping to increase compliance with information security policies was considered ineffective and had no significant impact. However, reducing reward significantly affected employee attitudes towards preventing lousy behaviour in information security [37]. The users in the university exhibited different characteristics from users in other organisations. Thus, the different desired rewards affected their behaviour to adhere to the policy consistently.

\section{Conclusion}

This study determines factors influencing the user to comply with information security policy at university. The results show that organisational commitment and habit users have a significant impact on compliance with information security policy. Organisational culture and reward variables represent factors that do not receive a considerable impact on users to always comply with information security policies. Contrary to previous research by Balliet (2011) says rewards have moderate to significant effects on compliance with information security policies [38. 
The results provide the idea that the organisation factors have contributed to user behaviour to comply with information security policies. This finding provides significant implications for exploring how to improve user compliance with information security, especially users at universities. The results cannot claim for all universally because this research is conducted explicitly for respondents from universities. Variety respondents willing to fill out the research questionnaire come from the lecturer, and officials who have interests will affect to find another factor that is influencing the user to comply with information security policy. Further research on the selection of respondent characteristics needs to consider obtaining significant results and following the facts in the field, like involving technical employees and students as active users of information systems at universities. This issue needs considering because information security is the responsibility of all levels of users, and all users must also comply with information security policies to fulfil the primary purpose of information security policy.

\section{References}

1. Morgan, S.: 2019 Official Annual Cybercrime Report next two decades. (2019).

2. Crossler, R.: An Extended Perspective on Individual Security Behaviors: Protection Motivation Theory and a Unified Security Practices ( USP ) Instrument. 45, 51-71 (2014).

3. Herath, T., Rao, H.R.: Protection motivation and deterrence: a framework for security policy compliance in organisations. Eur. J. Inf. Syst. 18, 106-125 (2009). https://doi.org/10.1057/ejis.2009.6.

4. Hwang, I., Kim, D., Kim, T., Kim, S.: Why not comply with information security? An empirical approach for the causes of non-compliance. Online Inf. Rev. 41, 2-18 (2017). https://doi.org/10.1108/OIR-11-2015-0358.

5. Ifinedo, P.: Information systems security policy compliance: An empirical study of the effects of socialisation, influence, and cognition. Inf. Manag. 51, 69-79 (2014). https://doi.org/10.1016/j.im.2013.10.001.

6. Alotaibi, M., Furnell, S., Clarke, N.: Information Security Policies : A review of Challenges and Influencing Factors. In: The 11th International Conference for Internet Technology and Secured Transactions. pp. 352-358 (2016).https://doi.org/10.1109/ICITST.2016.7856729.

7. D'Arcy, J., Greene, G.: Security culture and the employment relationship as drivers of employees' security compliance. Inf. Manag. Comput. Secur. 22, 474-489 (2014). https://doi.org/10.1108/IMCS-08-2013-0057.

8. Chang, A.J.T., Wu, C.Y., Liu, H.W.: The effects of job satisfaction and organisation commitment on information security policy adoption and compliance. In: 2012 IEEE 6th International Conference on Management of Innovation and Technology, ICMIT 2012. pp. 442-446 (2012). https://doi.org/10.1109/ICMIT.2012.6225846.

9. Gerber, N., McDermott, R., Volkamer, M., Vogt, J.: Understanding information security compliance - Why goal setting and rewards might be a bad idea. Int. Symp. Hum. Asp. Inf. Secur. Assur. (HAISA 2016). 10., 145-155 (2016).

10. Sommestad, T., Karlzén, H., Hallberg, J.: The Theory of Planned Behavior and Information Security Policy Compliance. J. Comput. Inf. Syst. 00, 1-10 (2017). https://doi.org/10.1080/08874417.2017.1368421.

11. NIST: Glossary of Key Information Security Terms [NISTIR 7298 Rev 2]. (2013). https://doi.org/10.1016/0735-6757(85)90039-7. 
12. Wright, C.: Assessing Security Awareness and Knowledge of Policy. In: The IT Regulatory and Standards Compliance Handbook. pp. 161-194 (2008).

13. Mowday, R.T.: Reflections on the study and relevance of organisational commitment. Hum. Resour. Manag. Rev. 8, 387-401 (1998). https://doi.org/10.1016/S1053-4822(99)00006-6.

14. Angle, H.L., Perry, J.L.: An Empirical Assess- ment of Organizational Commitment and Organizational Effective- ness. Adm. Sci. Q. 26, 1-14 (1981).

15. Brockner, J., Tyler, T.R., Cooper-Schneider, R.: The Influence of Prior Commitment to an Institution on Reactions to Perceived Unfairness: The Higher They Are, The Harder They Fall. Adm. Sci. Q. 37, 241 (1992). https://doi.org/10.2307/2393223.

16. Amin, M., Barati, O., Ghoroghchian, M.: Role of Organizational Climate in Organizational Commitment: The Case of Teaching Hospitals. Osong Public Heal. Res. Perspect. 7, 96100 (2016). https://doi.org/10.1016/j.phrp.2015.11.009.

17. Han, J.Y., Kim, Y.J., Kim, H.: An integrative model of information security policy compliance with psychological contract: Examining a bilateral perspective. Comput. Secur. 66, 52-65 (2017). https://doi.org/10.1016/j.cose.2016.12.016.

18. Alshare, K.A., Lane, P.L., Lane, M.R.: Information security policy compliance: a higher education case study. Inf. Comput. Secur. 26, 91-108 (2018). https://doi.org/10.1108/ICS09-2016-0073.

19. Arage, T., Belanger, F., Beshah, T.: Influence of National Culture on Employees' Compliance with Information Systems Security (ISS) Policies: Towards ISS Culture in Ethiopian Companies. In: AMCIS 2015 Proceedings. pp. 1-7 (2015).

20. Amankwa, E., Loock, M., Kritzinger, E.: Establishing information security policy compliance culture in organisations. Inf. Comput. Secur. 26, 420-436 (2018). https://doi.org/10.1108/ICS-09-2017-0063.

21. da Veiga, A., Astakhova, L. V., Botha, A., Herselman, M.: Defining organisational information security culture - Perspectives from academia and industry. Comput. Secur. 101713 (2020). https://doi.org/10.1016/j.cose.2020.101713.

22. Moody, G.D., Siponen, M., Pahnila, S.: Toward a Unified Model of Information Security $\begin{array}{llllll}\text { Policy Compliance. } & \text { MIS } & \text { Q. } & \text { 42, 285-311 }\end{array}$ https://doi.org/10.25300/MISQ/2018/13853.

23. Siponen, M., Adam Mahmood, M., Pahnila, S.: Employees' adherence to information security policies: An exploratory field study. Inf. Manag. 51, 217-224 (2014). https://doi.org/10.1016/j.im.2013.08.006.

24. Pahnila, S., Siponen, M., Mahmood, A.: Which Factors Explain Employees' Adherence to Information Security Policies? An Empirical Study. Pacis 2007 Proc. 438-439 (2007). https://doi.org/10.1007/978-0-387-72367-9_12.

25. Siponen, M., Pahnila, S., Mahmood, m. adam: Compliance with Information Security Policies: An Empirical Investigation. IEEE Comput. Soc. (2010).

26. Sommestad, T., Karlzén, H., Hallberg, J.: The Theory of Planned Behavior and Information Security Policy Compliance The Theory of Planned Behavior and Information Security Policy Compliance. J. Comput. Inf. Syst. 00, 1-10 (2017). https://doi.org/10.1080/08874417.2017.1368421.

27. Topa, I., Karyda, M.: From theory to practice: guidelines for enhancing information security management. Inf. Comput. Secur. 27, 326-342 (2019). https://doi.org/10.1108/ICS-092018-0108.

28. Hong, Y., Furnell, S.: Motivating Information Security Policy Compliance: Insights from Perceived Organizational Formalization. J. Comput. Inf. Syst. 00, 1-10 (2019). https://doi.org/10.1080/08874417.2019.1683781. 
29. Mowday, R.T., Steers, R.M., Porter, L.W.: The Masurement of Organizational Commitment: A Progress Report. J. Vocat. Behav. 14, 224-247 (1979).

30. Hogail, A. Al: Cultivating and assessing an organisational information security culture; an empirical study. Int. J. Secur. its Appl. 9, 163-178 (2015). https://doi.org/10.14257/ijsia.2015.9.7.15.

31. Martins, A., Eloff, J.: Information Security Culture. 191-201 (2002). https://doi.org/10.1007/978-0-387-35586-3_15.

32. Bulgurcu, B., Cavusoglu, H., Benbasat, I.: Information Security Policy Compliance: An Empirical Study of Rationality-Based Beliefs and Information Security Awareness. MIS Q. 34, 523-548 (2010). https://doi.org/10.1093/bja/aeq366.

33. Beckers, K., Cote, I., Fenz, S., Hatebur, D., Heisel, M.: A Structured Comparison of Security Standards. In: Engineering Secure Future Internet Services and Systems. pp. 1-34. Springer International Publishing, Switzerland (2014). https://doi.org/10.1007/978-3-319-07452-8.

34. Sue, V., Ritter, L.: Conducting Online Surveys. (2015). https://doi.org/10.4135/9781506335186.

35. Ringle, C.M., Sarstedt, M., Straub, D.: A critical look at the use of PLS-SEM in MIS Quarterly. MIS Q. 36, iii-xiv (2012). https://doi.org/10.3200/JOEB.79.4.213-216.

36. Alkalbani, A., Deng, H., Zhang, X.J.: Investigating the Impact of Institutional Pressures on Information Security Compliance in Organisations. Australas. Conf. Inf. Syst. 1-12(2016)

37. Safa, N.S., Maple, C., Furnell, S., Azad, M.A., Perera, C., Dabbagh, M., Sookhak, M.: Deterrence and prevention-based model to mitigate information security insider threats in organisations. Futur. Gener. Comput. Syst. 97, 587-597 (2019). https://doi.org/10.1016/j.future.2019.03.024.

38. Balliet, D., Mulder, L.B., M Van Lange, P.A., Lange, V., M, P.A.: Reward, Punishment, and Cooperation: A Meta-Analysis Citation. Psychol. Bull. 137, 594-615 (2011). https://doi.org/10.1037/a0023489. 

The $5^{\text {th }}$ International Conference of Reliable Information and Communication Technology (IRICT 2020)

$21^{\text {st }}-22^{\text {nd }}$ December 2020

(Virtual Conference)

“Innovative Systems for Intelligent Health Informatics "

Editors:

Faisal Saeed

Fathey Mohammed

Abdulaziz Al-Nahari 


\section{Organizing Committee}

\section{Honorary Co-Chairs}

Rose Alinda Alias, President, Association for Information Systems -Malaysian Chapter, Head of the Information Service Systems and Innovation Research Group (ISSIRG) in Universiti Teknologi Malaysia

Ahmad Lotfi, Head, Computing and Technology School of Science \& Technology, Nottingham Trent University (UK)

Abdullah Alsaeedi, Dean, College of Computer Science and EngineeringTaibah University, Kingdom of Saudi Arabia

\section{Conference General Chair}

Faisal Saeed, President, Yemeni Scientists Research Group (YSRG), Head of Data Science Research Group in Taibah University, Kingdom of Saudi Arabia

\section{Program Committee Chair}

Fathey Mohammed, Universiti Utara Malaysia (UUM), Malaysia

\section{General Secretary}

Nadhmi Gazem, Taibah University, Kingdom of Saudi Arabia

\section{Technical Committee Chair}

Faisal Saeed, Taibah University, Kingdom of Saudi Arabia

Tawfik Al-Hadhrami, Nottingham Trent University, UK

Mamoun Alazab, Charles Darwin University, Australia

\section{Publications Committee}

Fathey Mohammed, Universiti Utara Malaysia

Abdulaziz Al-Nahari, Sanaá Community College

\section{Publicity Committee}

Abdullah Aysh Dahawi, Universiti Teknologi Malaysia

Maged Naeser, Universiti Teknologi Malaysia

Mohammed Omar Awadh Al-Shatari, Universiti Teknologi PETRONAS

Ali Ahmed Ali Salem, Universiti Tun Hussein Onn Malaysia

\section{IT \& Multimedia Committee}

Fuad Abdeljalil Al-shamiri, Universiti Teknologi Malaysia

Mohammed Alsarem, Taibah University, KSA

Amer Alsaket, Sitecore, Malaysia

Sulaiman Mohammed Abdulrahman, Taibah University, KSA 


\section{Organizing Committee}

\section{Treasure Committee}

Abdullah Aysh Dahawi, Universiti Teknologi Malaysia

\section{Logistic Committee Chair}

Wahid Al-Twaiti, Universiti Teknologi Malaysia (UTM)

\section{Registration Committee Chair}

Sameer Hasan Albakri, Universiti Teknologi Malaysia (UTM) 


\section{International Technical Committee}

Abdelhadi Raihani, Hassan II University of Casablanca, Morocco

Abdelhamid Emara, Taibah University, KSA

Abdelkaher Ait Abdelouahad, Chouaib Doukkali University, Morocco

Abdualmajed Ahmed Ghaleb

Al-Khulaidi

Sana'a University, Yemen

Abdullah Almogahed,

University Utara Malaysia, Malaysia

Abdullah B Nasser,

University Malaysia Pahang, Malaysia

Abdulrahman Alsewari,

Abubakar Elsafi,

University Malaysia Pahang, Malaysia

Aby Mathews Maluvelil,

Ahmad Fadhil Yusof,

Ahmed Awad,

Ahmed Majid,

Ahmed Mutahar,

Ahmed Rakha,

Ahmed Talal,

Alaa Alomoush,

University of Jeddah, KSA

Independent Researcher, Canada

Universiti Technologi Malaysia, Malaysia

King Abdulaziz University, KSA

University of Information Technology and Communications, Iraq

Management \& Science University, Malaysia

Al-Azhar University, Eygpt

Al Iraqia University, Iraq

Universiti Malaysia Pahang, Malaysia

Alaa Fareed Abdulateef,

Ali Ahmed,

University Utara Malaysia, Malaysia

King Abdulaziz University, KSA

Ameen Ba Homaid,

University Malaysia Pahang, Malaysia

Aminu Aminu Mu'Azu,

Amr Tolba,

Amr Yassin,

Umaru Musa Yar'adua University Katsina, Nigria

King Saud University, KSA

Ibb University, Yemen

Anton Satria Prabuwono,

Arwa Aleryani,

King Abdulaziz University, KSA

Auday Hashim Saeed

Al-Wattar,

Bakar Ba Qatyan,

Bander Al-Rimy,

Bassam Al-Hameli,

Bouchaib Cherradi,

Mohammed Gamal

Alsamman,

Haitham Alali,

Ehsan Othman,

Eissa Alshari,

Fadhl Hujainah,

Faisal Saeed,

Independent Researcher, Canada

University of Mosul, Iraq

University Utara Malaysia, Malaysia

Unitar, Malaysia

University Malaysia Pahang, Malaysia

Hassan II University, Morocco

University Utara Malaysia, Malaysia

Emirates College of Technology, UAE

Ovgu Magdeburg, Germany

Ibb University, Yemen

University Malaysia Pahang, Malaysia

Taibah University, KSA

University Utara Malaysia, Malaysia

Independent Researcher, Oman

University Utara Malaysia, Malaysia

Universiti Technologi Malaysia, Malaysia

Al-Ahgaff University, Yemen

Universiti Sains Malaysia, Malaysia

University Utara Malaysia, Malaysia 
International Technical Committee

Hassan Silkan,

Hesham Alghodhaifi, Hiba Zuhair,

Hussein Abualrejal, Insaf BELLAMINE, Insaf Bellamine,

Jawad Alkhateeb,

Kamal Alhendawi,

Khairul Shafee, Kalid

Khaleel Bader Bataineh,

Khalili Tajeddine,

Marwa Alhadi,

Masud Hasan,

Mohamad Ghozali Hassan

Mohamed Abdel Fattah,

Mohamed Elhamahmy,

Mohammed A. Al-Shara

Mohammed Al-Mhiqani,

Mohammed Alsarem,

Mohammed Azrag,

Mohammed Nahid,

Mostafa Al-Emran,

Motasem Al Smadi,

Mustafa Ali Abuzaraida,

Mustafa Noori,

Nabil Al-Kumaim,

Nadhmi Gazem,

Naseebah Maqtary,

Nejood Hashim Al-Walidi,

Noor Akma,

Omar Dakkak,

Omar Zahour,

Osama Sayaydeh,

Qasim Alajmi,

Raed Aldhubhani,

Raghed Esmaeel,

Rajesh Kaluri,

Salah Abdelmageid,

Salwa Belaqziz,

Samar Ghazal,

Samar Salem Ahmed,

Sharaf J. Malebary,

Sinan Salih,

Soufiane Hamida,

Susan Abdulameer,
Université Chouaib Doukkali, Morocco

University of Michigan, US

Al-Nahrain University, Iraq

University Utara Malaysia, Malaysia

Chouaib Doukkali University, Morocco

FSDM Fès, Morocco

Taibah University, KSA

Alquds Open University, Palestine Universiti Teknologi PETRONAS, Malaysis

Amman Arab University, Jordan

Hassan II University of Casablanca, Morocco

Sana'a University, Yemen

Taibah University, KSA

University Utara Malaysia, Malaysia

Taibah University, KSA

Eygpt

University Malaysia Pahang, Malaysia

Universiti Teknikal Malaysia Melaka, Malaysia

Taibah University, KSA

University Malaysia Pahang, Malaysia

Hassan II University, Casablanca, Morocco

Buraimi University College, Oman

Jordan University of Science and Technology, Jordan

University Utara Malaysia, Malaysia

Middle Technical University, Iraq

Universiti Teknikal Malaysia Melaka, Malaysia

Taibah University, KSA

University of Science and Technology, Yemen

Cairo University, Egypt

Universiti Malaysia Pahang, Malaysia

UNIKA, Turkey

Hassan II University of Casablanca, Morocco

University Malaysia Pahang, Malaysia

A' Sharqiah University, Oman.

University of Hafr AlBatin, KSA

University of Mousl, Iraq

Vellore Institute of Technology, India

Taibah University, KSA

Ibn Zohr University, Morocco

Universiti Sains Malaysia, Malaysia

International Islamic University, Malaysia

King Abdulaziz University, KSA

Diglah University College, Iraq

Hassan II University of Casablanca, Morocco

Universiti Utara Malaysia, Malaysia 


\title{
International Technical Committee
}

\author{
Syifak Izhar Hisham, \\ Universiti Malaysia Pahang, Malaysia \\ Tawfik Al-Hadhrami, \\ Nottingham Trent University, UK \\ Waleed A. Hammood, \\ Universiti Malaysia Pahang, Malaysia \\ Waleed Ali, \\ King Abdulaziz University, KSA \\ Waleed Alomoush, \\ Imam Abdulrahman bin Faisal University, KSA \\ Waseem Alromimah, \\ Taibah University, KSA \\ Wasef Mater, \\ University of Petra, Jordan \\ Yaqoub Sulaiman, \\ King Abdulaziz University, KSA \\ Yousef Fazea, \\ University Utara Malaysia, Malaysia \\ Yousif Abdullah AlRashidi, Al Yamamah University, KSA \\ Yousif Aftan Abdullah, University of Baghdad, Iraq \\ Yousif Munadhil Ibrahim, University Utara Malaysia, Malaysia \\ Zainab Senan, \\ University Utara Malaysia, Malaysia
}




\section{Messages}

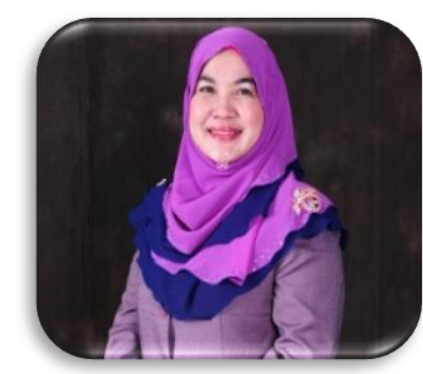

\section{CONFERENCE HONORARY CO- CHAIR MESSAGE}

I am pleased to welcome everyone to the 5th International Conference of Reliable Information and Communication Technology 2020 (IRICT 2020) that is held online from December 21 to December 22, 2020. IRICT 2020 is organized by the Yemeni Scientists Research Group (YSRG), in collaboration with Information Service Systems and Innovation Research Group (ISSIRG) in Universiti Teknologi Malaysia (Malaysia), School of Science \& Technology in Nottingham Trent (United Kingdom), College of Engineering, IT and Environment at Charles Darwin University (Australia), Data Science Research Group in Taibah University (Kingdom of Saudi Arabia), and Association for Information Systems Malaysia Chapter MyAIS (Malaysia).

IRICT 2020 is conducted online due to the global pendamic of COVID19. However this has not prevented our kind participants to actively contribute to IRICT 2020. We are happy that 140 papers have been submitted by researchers from 29 countries including Algeria, Australia, China, Egypt, Fiji, Germany, India, Indonesia, Iraq, Iran, Jordon, Malaysia, Morocco, Myanmar, Nigeria, Oman, Pakistan, Saudi Arabia, Singapore, Somalia, South Africa, Sri Lanka, Sudan, Sweden, Taiwan, Tunisia, United Kingdom, United States and Yemen. Of those 140 submissions, 111 submissions have been selected to be included in conference and subsequent book publication. All submissions underwent a rigorous doubleblind peer-review process. The conference proceedings will be published in Springer Book Series: "Advances in Intelligent Systems and Computing". The IRICT 2020 program involves six keynote speeches and three parallel sessions during the two days of the conference.

I would like to thank all authors for the participation in IRICT 2020. Many thanks go to our keynote speakers, Prof. Dr. Samir Abou El-Seoud (the British University in Egypt, Egypt), Prof. Dr. Mischa Dohler (King's College London, United Kingdom), Prof. Dr. Zeeshan Pervez (University of the West of Scotland (UWS), United Kingdom), Assoc. Prof. Dr. Noorminshah Iahad (Universiti Teknologi Malaysia (UTM), Malaysia), Dr. Monther Alhamdoosh (The University of Melbourne, Australia) and Dr. Mustapha HAMDI (National Institute of Applied Science and Technology, Tunisia) for sharing their knowledge and experience with us in IRICT 2020. Our utmost appreciation goes to the dedicated teams, the organizing and technical committees for the great efforts in making this conference a huge success!

I hope you are enjoying the conference this year, albeit it is from your own home and I wish you every success in your research endeavours.

Prof. Dr. Rose Alinda Alias

IRICT 2020 Honorary Co-Chair

President,Association for Information Systems - Malaysian Chapter

Head, Information Service Systems and Innovation Research Group (ISSIRG) 


\section{Messages}

\section{CONFERENCE HONORARY CO- CHAIR MESSAGE}

I would like to welcome you to the $5^{\text {th }}$ International Conference of Reliable Information and Communication Technology 2020 (IRICT 2020).

We are happy to collaborate with our colleagues from Yemen, Malaysia, Saudi Arabia and Australia in ogranising this conference and discuss one of the main challenges worldwide in the field of Intelligent Health Informatics. I would like to thank our great committee from the Yemeni Scientists Research Group (YSRG), Information Service Systems and Innovation Research Group (ISSIRG) in Universiti Teknologi Malaysia (Malaysia), School of Science \& Technology in Nottingham Trent (United Kingdom), College of Engineering, IT and Environment at Charles Darwin University (Australia), Data Science Research Group in Taibah University (Kingdom of Saudi Arabia), and Association for Information Systems -Malaysia Chapter MyAIS (Malaysia).

Since 2018, our team members at the School of Science and Technology in Nottingham Trent University (NTU) participated in the technical committee of this conference and presented some findings of our research projects. This year, we have an exciting program at IRICT 2020 that will allow members to reflect upon and celebrate our past accomplishments, and use the online platform to renew friendships and extend our networks. In IRIC 2020, we will jointly explore current and future research directions in intelligent health informatics that contribute significantly toward serving and protecting our societies.

Finally, I would like to express my sincere gratitude to the keynote speakers and the conference participants for their contributions and I wish you a great time in the conference!

Prof. Dr. Ahmad Lotfi

IRICT 2020 Honorary Co-Chair

Head of Computing and Technology

School of Science and Technology

Nottingham Trent University, United Kingdom 


\title{
Messages
}

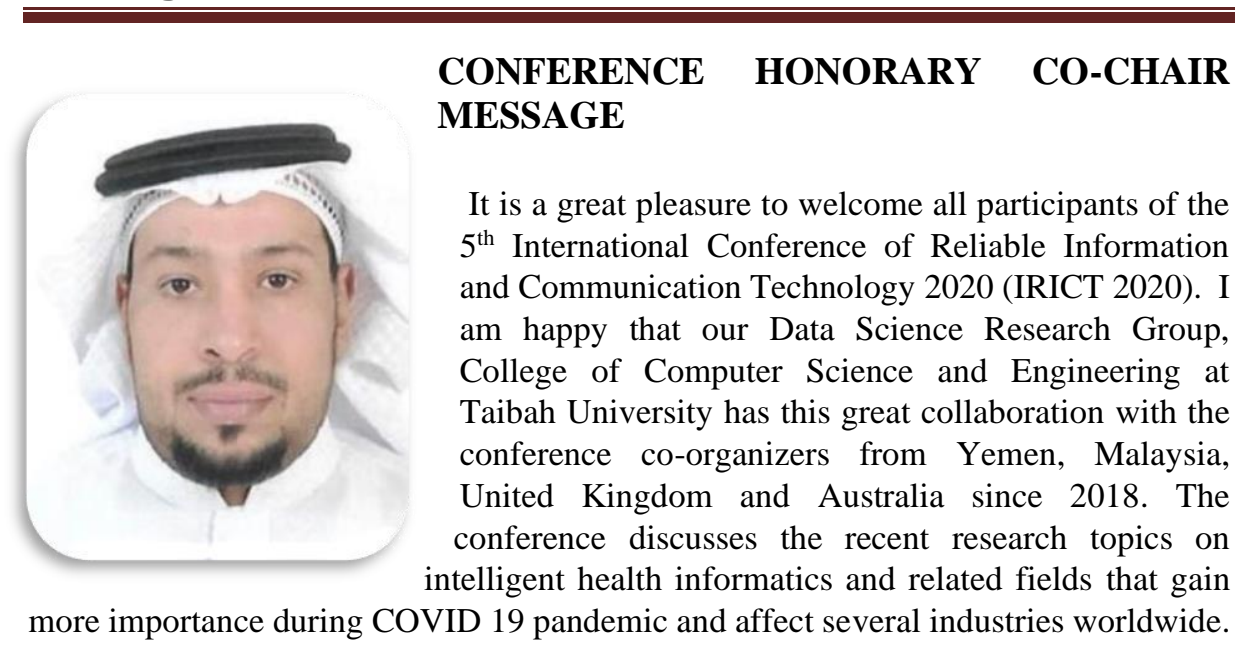

The theme of IRICT 2020 conference is "Innovative Systems for Intelligent Health Informatics" that is aligned with our research interests in Taibah University. We are conducting several projects on COVID 19 in different research disciplines such as Computing, Medicine, Social Sciences, Education and Engineering.

IRICT 2020 received 140 papers from several countries. We are happy that our researchers in Taibah University participated in this conference and will share some findings of their researches. In addition, our team has actively worked in the organizing and technical committee of IRICT 2020 along with our colleagues from Yemeni Scientists Research Group (YSRG), Information Service Systems and Innovation Research Group in Universiti Teknologi Malaysia (Malaysia), School of Science \& Technology in Nottingham Trent (United Kingdom), College of Engineering, IT and Environment at Charles Darwin University (Australia), and Association for Information Systems - Malaysia Chapter (Malaysia).

I would like to thank the organizing and technical committee members and congratulate them for the great success of this conference. Many thanks to our keynote speakers and participants of IRICT 2020 conference for sharing their knowledge and research findings with us.

I wish you all the best and hopefully we can meet in person during IRICT 2021.

\author{
Dr. Abdullah Alsaeedi \\ IRICT 2020 Honorary Co-Chair \\ Dean, College of Computer Science and Engineering \\ Taibah University, Kingdom of Saudi Arabia
}




\title{
Messages
}

\section{CONFERENCE MESSAGE \\ GENERAL CHAIR}

I would like to welcome all of you to the $5^{\text {th }}$ International Conference of Reliable Information and Communication Technology 2020 (IRICT 2020) that is held online on 21-22, December 2020.

The conference book includes 111 papers that discuss several research topics such as health informatics, bioinformatics, artificial intelligence, machine learning, data mining, big data, business intelligence, internet of things, communication systems, Information Security, computational vision and robotics, information Systems and advances in software engineering. These papers will be presented in three parallel sessions during the two days.

I would like to thank the organizing and technical committee members for their great efforts in ensuring the successful implementation of the conference. In particular, I would like to thank our Honorary Co-Chairs, Prof. Dr. Rose Alinda Alias, President of Association for Information Systems - Malaysian Chapter and Head of Information Service Systems and Innovation Research Group (ISSIRG); Prof. Dr. Ahmad Lotfi, Professor of Computational Intelligence, Nottingham Trent University, Nottingham, UK; Dr. Abdullah Alsaeedi Dean, College of Computer Science and Engineering Taibah University, Kingdom of Saudi Arabia; Dr. Fathey Mohammed, Program and Publication Committee Chair; Dr. Sameer Albakri, Registration Committee Chair; Dr. Nadhmi Gazem, General Secretary; Dr. Tawfik Al-Hadrami, Technical Committee CoChair; Abdullah Dahawi, Finance Committee Chair and all other members. I am very happy and proud to work with a great team like you. Also, I would like to thank our keynote speakers for sharing their knowledge and expertise with us, and to all authors who contributed significantly to this event. Many thanks to Prof. Dr. Janusz Kacprzyk, AISC series editor; Dr. Thomas Ditzinger and Nareshkumar Mani from Springer.

Finally, I hope you enjoy attending IRICT 2020 conference from home and I hope you are staying healthy and safe.

\author{
Dr. Faisal Saeed \\ IRICT 2020 General Chair \\ President, Yemeni Scientists Research Group (YSRG) \\ Head of Data Science Research Group \\ Taibah University, Kingdom of Saudi Arabia
}




\section{Messages}

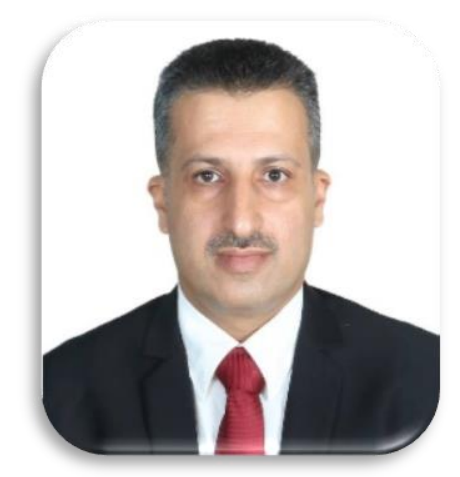

\section{CONFERENCE PROGRAM CHAIR MESSAGE}

It is my great pleasure to welcome you to the $5^{\text {th }}$ International Conference of Reliable Information and Communication Technology (IRICT 2020). This book presents 111 abstracts of the papers that are scheduled to be presented online on 21-22, December 2020. The full papers are included in IRICT 2020 Proceedings which is submitted to Springer to be published in "Advances in Intelligent Systems and Computing" Book Series.

I would like to thank all authors for submitting their research works to IRICT 2020 and for presenting and discussing their papers during the conference. I also would like to express my gratitude to the external reviewers for providing constructive and valuable suggestions for the authors to improve the quality of the papers.

Also, I would like to take this opportunity to express my utmost gratitude and sincere thanks and appreciation to Dr. Abdulaziz Al-Nahari, the Publication Committee Cochair for his great efforts in preparing the Springer required documents and editing this book as well.

I also would like to extend our thanks and appreciation to all who participated, attended and supported this conference and make it a reality.

Finally, I hope that all guests, participants, and attendees have a great time at IRICT 2020. The success of this conference will inspire us more in future.

Dr. Fathey Mohammed,

Program Chair, IRICT 2020 


\section{Keynote Speakers}

\section{Keynote Speaker I:}

Samir Abou El-Seoud

Professor at The British University in Egypt (BUE)

\section{Keynote title:}

\section{"Leukemia Cells Detection and Classification Using Convolutional Neural Network"}

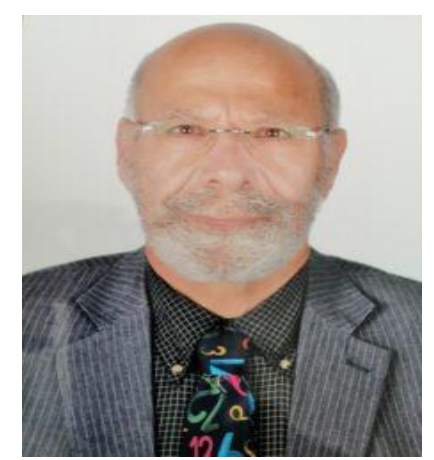

\section{Brief Profile}

Prof. Dr. Samir Abou El-Seoud received his BSc degree in Physics, Electronics and Mathematics from Cairo University in 1967, his Higher Diplom in Computing from Technical University of Darmstadt (TUD) /Germany in 1975 and his Doctor of Science from the same University (TUD) in 1979. Field of study: Scientific Computations and Parallel Algorithms. Research interests: Computer Aided Learning, Parallel Algorithms, Mobile Applications, Augmented Reality, Cloud Computing, IoT, Numerical Scientific Computations and Computational Fluid Mechanics. Teaching Interest: Computer Graphics, Computer Organization, Computer Architecture, Logic and Digital Design, Analysis and Design of Algorithms, Parallel Algorithms, and Data Structure. Professor El-Seoud helds different academic positions at TUD Germany. Letest Full-Professor in 1987. Outside Germany Professor El-Seoud spent different years as a Full-Professor of Computer Science at SQU - Oman, Qatar University, and PSUT-Jordan and acted as a Head of Computer Science for many years. At industrial institutions, Professor El-Seoud worked as Scientific Advisor and Consultant for the GTZ in Germany and was responsible for establishing a postgraduate program leading to M.Sc. degree in Computations at Colombo University / Sri-Lanka (2001 - 2003). He also worked as Application Consultant at Automatic Data Processing Inc., Division Network Services in Frankfurt/Germany (1979 - 1980). Professor El-Seoud joined The British University in Egypt (BUE) in 2012. Currently, he is Basic Science Coordinator at the Faculty of Informatics and Computer Science (ICS) at BUE. Professor El-Seoud has more than 150 publications in international proceedings and international reputable journals. 


\section{Keynote Speakers}

Keynote Speaker II:

Mischa Dohler

Professor at Faculty of Natural \& Mathematical Sciences King's College London, United Kingdom

Keynote title:

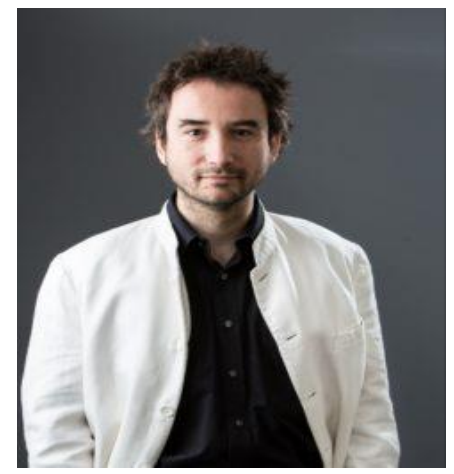

"The Immersive World of $6 G$ "

\section{Brief Profile}

Prof. Dr. Mischa Dohler is a full Professor in Wireless Communications at King's College London, driving cross-disciplinary research and innovation in technology, sciences and arts. He is a Fellow of the IEEE, the Royal Academy of Engineering, the Royal Society of Arts (RSA), the Institution of Engineering and Technology (IET); and a Distinguished Member of Harvard Square Leaders Excellence. He is a serial entrepreneur with 5 companies; composer \& pianist with 5 albums on Spotify/iTunes; and fluent in 6 languages. He acts as policy advisor on issues related to digital, skills and education. He has had ample coverage by national and international press and media. $\mathrm{He}$ is a frequent keynote, panel and tutorial speaker, and has received numerous awards. He has pioneered several research fields, contributed to numerous wireless broadband, IoT/M2M and cyber security standards, holds a dozen patents, organized and chaired numerous conferences, was the Editor-in-Chief of two journals, has more than 300 highly-cited publications, and authored several books. He was the Director of the Centre for Telecommunications Research at King's from 2014-2018. He is the Cofounder of the Smart Cities pioneering company Worldsensing, where he was the CTO from 2008-2014. He also worked as a Senior Researcher at Orange/France Telecom from 2005-2008. 


\section{Keynote Speakers}

\section{Keynote Speaker III:}

Noorminshah Iahad

Associate Professor at Universiti Teknologi Malaysia (UTM), Malaysia

Johor, Malaysia

Keynote title:

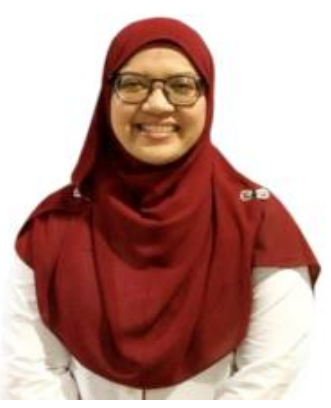

"Emerging Trends in Internet of Health Things"

\section{Brief Profile}

Associate Professor Dr Noorminshah A.Iahad graduated from the University of Manchester in 2008. She has supervised Masters and PhD students in the areas such as Health Informatics, Gamification, E-learning, Mobile learning and Computer Supported Project Based Learning. Nine PhD students and 4 Masters students have graduated under her supervision. She has led various research projects and won 1 silver medal awards at UTM level, 1 gold award at the National level and 1 Silver Medal Ward at the International level. Her current research interest is on IT as a catalyst for consumer wellness informatics and business digital transformation. She is currently the IT Manager at the office of Deputy Vice Chancellor (Research \& Innovation). She was the Secretary of Malaysia Association for Information Systems for one term and is a member of IEEE. 


\section{Keynote Speakers}

\section{Keynote Speaker IV:}

\section{Zeeshan Pervez}

Professor at the University of the West of Scotland (UWS), United Kingdom

\section{Keynote title:}

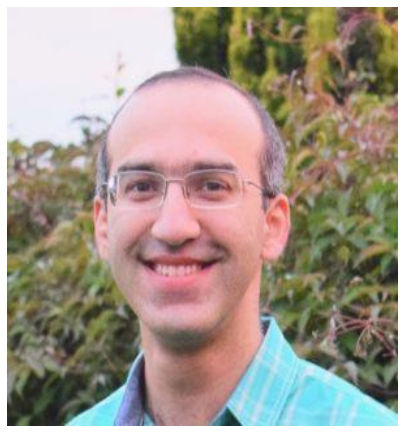

"User privacy and infrastructure security for IoT and 5G Networks"

\section{Brief Profile}

Dr. Zeeshan Pervez is a Professor at the University of the West of Scotland (UWS). $\mathrm{He}$ is a Senior Member of IEEE, ACM Distinguished Speaker, Member ISO and BSI working groups for FM sector, Fellow of Higher Education Academy (UK), and Full Member of EPSRC Peer Review College (UK).

He has a strong track record of securing research, industry, and capacity building funding from the Innovate UK, Scottish Funding Council, Innovation Centres, European Commission, Erasmus+, Microsoft Research, and international research institutes.

His research interests are Internet-of-Things, Cybersecurity, Applied AI/ML, Blockchain Technologies, Data Security \& Privacy, and Cloud Computing. The application areas of his research are but not limited to Industry 4.0, Smart Cities, Social Housing, Predictive Maintenance, Facilities Management, Healthcare, Data Science, and ICT4D. $\mathrm{He}$ has successfully supervised several postdoctoral fellows, postgraduate research/taught and undergraduate students. He has published over $80 \mathrm{SCI} / \mathrm{E}$ indexed journals and IEEE/ACM conferences. He has served as a TPC member of more than 100 conferences and a regular reviewer of 30+ IEEE Transactions, Elsevier, and Springer journals. He has chaired and evaluated several doctoral exams for the UK and international higher education institutes.

He has been regularly invited by national and international research and academic institutes to deliver talks on topic ranging from Internet-of-Things, Data Science, Cybersecurity, and Cloud Computing. 


\section{Keynote Speakers}

\section{Keynote Speaker V:}

\section{Mustapha HAMDI}

Associate Professor at INSAT. Tunisia

\section{Keynote title:}

"Industry 4.0 architecture From Edge AI to Cloud"

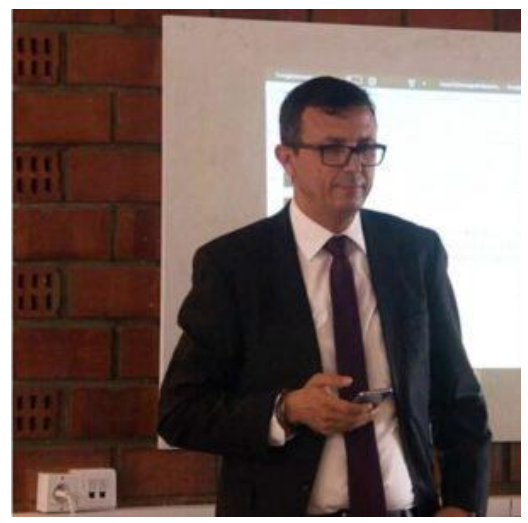

\section{Brief Profile}

Mustapha HAMDI is an Associate Professor at INSAT, Tunisia. His main academic activities are:

1) Internet of Things: Nodes Low Layer Programming, BLE, LoRaWAN, Node-RED, 2) Cloud and AI: Visual Recognition, Virtual Assistant, Machine Learning and Deep Learning

3) Codesign: Partitioning HW, SW, Implementaion on Altera and Xlinx platform

SoC architecture: advanced processors, embedded C, embedded buses "AHB, APB ..", management of interrupts "NVIC", GPIO, TIMERs, DAC / ADC. The main research activities are:

I) IoT: Codesign, reconfigurable IP, ARM SoC, optimization

II) Edge computing

III) Nanorobotic thesis: Characterization design, multiphysics and multi-scale modeling, molecular dynamics, semi-empirical methods, quantum physics. He also has about twenty publications. 


\section{Keynote Speakers}

\section{Keynote Speaker VI:}

Monther Alhamdoosh

Senior Scientist, Senior Manager Bioinformatics \& AI (CSL Limited)

Honorary Research Fellow (The University of Melbourne), Australia

\section{Keynote title:}

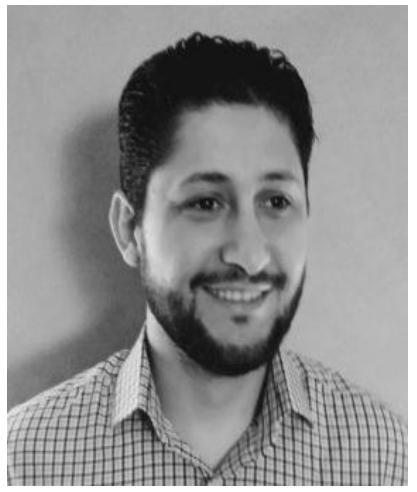

"The role of genomics and AI in advancing healthcare"

\section{Brief Profile}

Monther obtained a PhD in Machine Learning and Bioinformatics from La Trobe University, Australia, an MSc in Bioinformatics from the University of Bologna, Italy, and a BEng in Informatics (with a major in AI\&NLP) from University of Aleppo, Syria. He pursued a very successful academic career where he received several distinction awards and scholarships for his outstanding performance. He has published journal and conference articles and authored a book on the theory of machine learning and its applications in bioinformatics. His research interests include computational genomics, translational / clinical bioinformatics, computational intelligence, and IoT healthcare. At CSL, Monther heads the Bioinformatics and Artificial Intelligence group in the Global Research Department where his team contributes to solving cutting-edge problems in the biomedical field. His group works very closely with teams at CSL Research to identify new biomarkers and understand the mechanism of action of drug targets and diseases with a focus on improving patients' lives. Monther is an Honorary Fellow of The University of Melbourne. 


\section{Conference Program}

\begin{tabular}{|c|c|c|c|}
\hline Day 1: & \multicolumn{3}{|c|}{ Monday 21st December, 2020} \\
\hline \multicolumn{4}{|c|}{$\begin{array}{l}\text { Zoom Meeting ID: } \mathbf{8 6 8 8 4 4 2} 1517 \\
\text { Zoom Passcode } \quad \text { : IRICT2020 }\end{array}$} \\
\hline Session 1 & \multicolumn{3}{|c|}{ Main Room } \\
\hline $7: 10$ am - 7:15 am & \multicolumn{3}{|l|}{ Doa'a Recitation } \\
\hline $7: 15$ am - 7:30 am & \multicolumn{3}{|c|}{$\begin{array}{l}\text { Welcoming speech by Dr. Faisal Saeed, the IRICT2020 General } \\
\text { Chair }\end{array}$} \\
\hline $7: 30$ am - 7:45 am & \multicolumn{3}{|c|}{$\begin{array}{l}\text { Welcoming speech by Prof. Dr. Rose Alinda Alias, IRICT } \\
2020 \text { Honorary Chair }\end{array}$} \\
\hline $7: 45 a m-8: 30 a m$ & \multicolumn{3}{|c|}{$\begin{array}{l}\text { Keynote Speech } 1 \text { by: Prof. Dr. Samir Abou El-Seoud } \\
\text { Title: Leukemia Cells Detection and Classification Using Con- } \\
\text { volutional Neural Network }\end{array}$} \\
\hline $8: 30 a m-9: 15 a m$ & \multicolumn{3}{|c|}{$\begin{array}{l}\text { Keynote Speech } 2 \text { by: Prof. Dr. Mischa Dohler } \\
\text { Title: The Immersive World of } 6 G\end{array}$} \\
\hline 9:15 am - 9:30 am & \multicolumn{3}{|c|}{ Break } \\
\hline \multirow[t]{3}{*}{$9: 30 \mathrm{am}-12: 00 \mathrm{pm}$} & \multicolumn{3}{|c|}{ Parallel Session 1} \\
\hline & $\begin{array}{l}\text { Main Room } \\
\text { (Artificial Intel- } \\
\text { ligent) }\end{array}$ & $\begin{array}{l}\text { Room } 1 \\
\text { (Data Science and Big } \\
\text { Data Analytics) }\end{array}$ & $\begin{array}{l}\text { Room } 2 \\
\text { (Information } \\
\text { System) }\end{array}$ \\
\hline & 10 papers & 10 papers & 10 papers \\
\hline $12: 00 \mathrm{pm}-12: 15 \mathrm{pm}$ & \multicolumn{3}{|c|}{ Break } \\
\hline Session 2 & \multicolumn{3}{|c|}{ Main Room } \\
\hline $12: 15 \mathrm{pm}-1: 00 \mathrm{pm}$ & \multicolumn{3}{|c|}{$\begin{array}{l}\text { Keynote Speech } 3 \text { by: Prof. Dr. Zeeshan Pervez } \\
\text { Title: User privacy and infrastructure security for IoT and } 5 G \\
\text { Networks }\end{array}$} \\
\hline \multirow[t]{3}{*}{$1: 00 \mathrm{pm}-3: 45 \mathrm{pm}$} & \multicolumn{3}{|c|}{ Parallel Session 2} \\
\hline & $\begin{array}{l}\text { Main Room } \\
\text { (Artificial Intel- } \\
\text { ligent) }\end{array}$ & $\begin{array}{l}\text { Room } 1 \\
\text { (Data Science and Big } \\
\text { Data Analytics) }\end{array}$ & $\begin{array}{c}\text { Room } 2 \\
\text { (Information } \\
\text { Security) }\end{array}$ \\
\hline & 11 papers & 11 papers & 11 papers \\
\hline
\end{tabular}




\section{Conference Program}

\begin{tabular}{|c|c|c|c|}
\hline Day 2: & \multicolumn{3}{|c|}{ Tuesday 22nd December, 2020} \\
\hline \multicolumn{4}{|c|}{$\begin{array}{l}\text { Zoom Meeting ID: } \mathbf{8 8 9} 8676 \mathbf{6 2 4 8} \\
\text { Zoom Passcode }: \text { IRICT2020 }\end{array}$} \\
\hline Session 1 & \multicolumn{3}{|c|}{ Main Room } \\
\hline $8: 00 \mathrm{am}-8: 45 \mathrm{am}$ & \multicolumn{3}{|c|}{$\begin{array}{l}\text { Keynote Speech } 4 \text { by: Assoc. Prof. Dr. Noorminshah Iahad } \\
\text { Title: Emerging Trends in Internet of Health Things }\end{array}$} \\
\hline $8: 45$ am - 9:30 am & \multicolumn{3}{|c|}{$\begin{array}{l}\text { Keynote Speech } 5 \text { by: Dr. Monther Alhamdoosh } \\
\text { Title: The role of genomics and AI in advancing healthcare }\end{array}$} \\
\hline 9:30 am - 9:45 am & \multicolumn{3}{|c|}{ Break } \\
\hline \multirow[t]{3}{*}{$9: 45$ am - 11:45 am } & \multicolumn{3}{|c|}{ Parallel Session 1} \\
\hline & $\begin{array}{c}\text { Main Room } \\
\text { (Artificial Intel- } \\
\text { ligent) }\end{array}$ & $\begin{array}{c}\text { Room } 1 \\
\text { (Data Science and Big } \\
\text { Data Analytics) }\end{array}$ & $\begin{array}{c}\text { Room } 2 \\
\text { (Information } \\
\text { System) }\end{array}$ \\
\hline & 8 papers & 8 papers & 8 papers \\
\hline $11: 45 \mathrm{am}-12: 00 \mathrm{pm}$ & \multicolumn{3}{|c|}{ Break } \\
\hline Session 2 & \multicolumn{3}{|c|}{ Main Room } \\
\hline $\begin{array}{l}\text { 12:00 pm - 12:45 } \\
\text { pm }\end{array}$ & \multicolumn{3}{|c|}{$\begin{array}{l}\text { Keynote Speech } 6 \text { by: Dr. Mustapha HAMDI } \\
\text { Title: Industry } 4.0 \text { architecture From Edge AI to Cloud }\end{array}$} \\
\hline \multirow[t]{3}{*}{$12: 45 \mathrm{pm}-2: 45 \mathrm{pm}$} & \multicolumn{3}{|c|}{ Parallel Session 2} \\
\hline & $\begin{array}{c}\text { Main Room } \\
\text { (Computational } \\
\text { Vision + Tele- } \\
\text { communication) }\end{array}$ & $\begin{array}{c}\text { Room } 1 \\
\text { (Software Engineer- } \\
\text { ing) }\end{array}$ & $\begin{array}{l}\text { Room } 2 \\
\text { (Telecommuni- } \\
\text { cation and IoT) }\end{array}$ \\
\hline & 8 papers & 8 papers & 8 papers \\
\hline $2: 45 \mathrm{pm}-3: 00 \mathrm{pm}$ & \multicolumn{3}{|c|}{ Closing ceremony } \\
\hline
\end{tabular}


Sessions Schedule

\begin{tabular}{|c|c|}
\hline \multicolumn{2}{|r|}{ Monday, $2^{\text {st }}$ December, 2020} \\
\hline \multicolumn{2}{|r|}{ Parallel Session I } \\
\hline Time & 9:30am - 12:00 pm (GMT time) \\
\hline Zoom link : & $\begin{array}{l}\text { https://us02web.zoom.us/j/86884421517?pwd } \\
\text { =aTNma1dCVmloSXZ0eVk2OEdXYVmQT09 }\end{array}$ \\
\hline Meeting ID : & 86884421517 \\
\hline Passcode : & IRICT2020 \\
\hline \multicolumn{2}{|c|}{ Main Room (Artificial Intelligence) } \\
\hline $9: 30$ am - 9:45 am & $\begin{array}{l}\text { An Architecture for Intelligent Diagnosing Dia- } \\
\text { betic Types and Complications Based on Symp- } \\
\text { toms } \\
\begin{array}{l}\text { Gunasekar Thangarasu, P.D.D. Dominic and } \\
\text { Kayalvizhi Subramanian }\end{array}\end{array}$ \\
\hline 9:45 am - 10:00 am & $\begin{array}{l}\text { UPLX: Blockchain Platform for Integrated Health } \\
\text { Data Management } \\
\text { Omar Musa1, Lim Shu Yun, Reza Ismail }\end{array}$ \\
\hline 10:00 am - 10:15 am & $\begin{array}{l}\text { Detection of Cardiovascular Disease using Ensem- } \\
\text { ble Machine Learning Techniques } \\
\text { Fizza Kashif and Umi Kalsom Yusof }\end{array}$ \\
\hline 10:15 am - 10:30 am & $\begin{array}{l}\text { Intelligent Health Informatics with Personalisa- } \\
\text { tion in Weather-based Healthcare using Machine } \\
\text { Learning } \\
\text { Radiah Haque, Sin-Ban Ho, Ian Chai, Chin-Wei Teoh1, } \\
\text { Adina Abdullah, Chuie-Hong Tan, and Khairi Shazwan } \\
\text { Dollmat }\end{array}$ \\
\hline $10: 30$ am - 10:45 am & $\begin{array}{l}\text { Effective Web Service Classification Using a Hy- } \\
\text { brid of Ontology Generation and Machine Learn- } \\
\text { ing Algorithm } \\
\text { Murtoza Monzur, Radziah Mohamad and Nor Azizah } \\
\text { Saadon }\end{array}$ \\
\hline
\end{tabular}


Sessions Schedule

\begin{tabular}{|c|c|}
\hline 10:45 am - 11:00 am & $\begin{array}{l}\text { SMARTS D4D Application Module for Dietary Ad- } \\
\text { herence Self-Monitoring among Hemodialysis Pa- } \\
\text { tients } \\
\text { Hafzan Yusoff Nur Intan Raihana Ruhaiyem and Mohd } \\
\text { Hakim Zakaria }\end{array}$ \\
\hline 11:00 am - 11:15 am & $\begin{array}{l}\text { Improved Multi-Label Medical Text Classification } \\
\text { using Features Cooperation } \\
\text { Rim Chaib, Nabiha Azizi, Nawel Zemmal, Didier } \\
\text { Schwab and Samir Brahim Belhaouari }\end{array}$ \\
\hline 11:15 am - 11:30 am & $\begin{array}{l}\text { Image Modeling through Augmented Reality for } \\
\text { Skin Allergies Recognition } \\
\text { Nur Intan Raihana Ruhaiyem and Nur Amalina } \\
\text { Mazlan }\end{array}$ \\
\hline 11:30 am - 11:45 am & $\begin{array}{l}\text { Hybridisation of Optimised Support Vector Ma- } \\
\text { chine and Artificial Neural Network for Diabetic } \\
\text { Retinopathy Classification } \\
\text { Nur Izzati Ab Kader, Umi Kalsom Yusof and Maziani } \\
\text { Sabudin }\end{array}$ \\
\hline 11:45 am - 12:00 pm & $\begin{array}{l}\text { Binary Cuckoo Optimisation Algorithm and Infor- } \\
\text { mation Theory for Filter-Based Feature Selection } \\
\text { Ali Muhammad Usman, Umi Kalsom Yusof and Syi- } \\
\text { brah Naim }\end{array}$ \\
\hline \multicolumn{2}{|c|}{ Room 1 (Data Science and Big Data Analytics) } \\
\hline 9:30 am - 9:45 am & $\begin{array}{l}\text { A Habit-Change Support Web-Based System with } \\
\text { Big Data Analytical Features for Hospitals (Doc- } \\
\text { tive) } \\
\text { Cheryll Anne Augustine and Pantea Keikhosrokiani }\end{array}$ \\
\hline 9:45 am - 10:00 am & $\begin{array}{l}\text { Big Data Interoperability Framework for Malay- } \\
\text { sian Public Open Data } \\
\text { Najhan Muhamad Ibrahim, Amir Aatieff Amir Hussin, } \\
\text { Khairul Azmi Hassan and Ciara Breathnach }\end{array}$ \\
\hline
\end{tabular}


Sessions Schedule

\begin{tabular}{|c|c|}
\hline 10:00 am - 10:15 am & $\begin{array}{l}\text { Financial Time Series Forecasting Using Prophet } \\
\text { Umi Kalsom Yusof, Mohd Nor Akmal Khalid, Abir } \\
\text { Hussain and Haziqah Shamsudin }\end{array}$ \\
\hline 10:15 am - 10:30 am & $\begin{array}{l}\text { Open Data in Prediction using Machine Learning: } \\
\text { A Systematic Review } \\
\text { Norismiza Ismail and Umi Kalsom Yusof }\end{array}$ \\
\hline $10: 30$ am - 10:45 am & $\begin{array}{l}\text { Big Data Analytics Based Model for Red Chili } \\
\text { Agirculture in Indonesia } \\
\text { Junita Juwita Siregar and Arif Imam Suroso }\end{array}$ \\
\hline 10:45 am - 11:00 am & $\begin{array}{l}\text { Extracting Semantic Concepts and Relations from } \\
\text { Scientific Publications by Using Deep Learning } \\
\text { Fatima N. AL-Aswadi, Huah Yong Chan and Keng } \\
\text { Hoon Gan }\end{array}$ \\
\hline 11:00 am - 11:15 am & $\begin{array}{l}\text { Performance Degradation of Multi-class Classifi- } \\
\text { cation Model due to Continuous Evolving Data } \\
\text { Streams } \\
\text { Abdul Sattar Palli, Jafreezal Jaafar, Manzoor Ahmed } \\
\text { Hashmani }\end{array}$ \\
\hline 11:15 am - 11:30 am & $\begin{array}{l}\text { Landmark Localization in Occluded Faces Using } \\
\text { Deep Learning Approach } \\
\text { Zieb Rabie Alqahtani, Mohd Shahrizal Sunar, } \\
\text { Abdulaziz A. Alashbi }\end{array}$ \\
\hline $11: 30$ am - 11:45 am & $\begin{array}{l}\text { A Fusion Schema of Hand-Crafted Feature and } \\
\text { Feature Learning for Kinship Verification } \\
\text { Mohammed Ali Almuashi, Siti Zaiton Mohd Hashim, } \\
\text { Nooraini Yusoff, Khairul Nizar Syazwan }\end{array}$ \\
\hline $11: 45$ am - 12:00 pm & $\begin{array}{l}\text { A Terms Interrelationship Approach to Query } \\
\text { Expansion Based on Terms Selection } \\
\text { Nuhu Yusuf, Mohd Amin Mohd Yunus, Norfaradilla } \\
\text { Wahid, Mohd Najib Mohd Saleh, and Aida Mustapha }\end{array}$ \\
\hline
\end{tabular}


Sessions Schedule

\begin{tabular}{|l|l|}
\hline \multicolumn{2}{|c|}{ Room 2 (Information System) } \\
\hline 9:30 am - 9:45 am & $\begin{array}{l}\text { Derivation of Factors in Dealing Negative E-WOM } \\
\text { for Maintaining Online Reputation } \\
\text { Rizka Dhini Kurnia, Halina Mohamed Dahlan, } \\
\text { Samsuryadi }\end{array}$ \\
\hline 9:45 am - 10:00 am & $\begin{array}{l}\text { Trusted Factors of Social Commerce Product Re- } \\
\text { view Video } \\
\text { Humaira Hairudin Halina Mohamed Dahlan Ahmad } \\
\text { Fadhil Yusof }\end{array}$ \\
\hline 10:00 am - 10:15 am & $\begin{array}{l}\text { Exploring the Influence of Human-centered De- } \\
\text { sign on User Experience in Health Informatics } \\
\text { Sector: A Systematic Review } \\
\text { Lina Fatini Azmi and Norasnita Ahmad }\end{array}$ \\
\hline 10:15 am - 10:30 am & $\begin{array}{l}\text { An Emotional-Persuasive Habit-Change Support } \\
\text { Mobile Application for Heart Disease Patients } \\
\text { (BeHabit) } \\
\text { Bhavani Devi Ravichandran and Pantea } \\
\text { Keikhosrokiani }\end{array}$ \\
\hline 11:00 am - 11:15 am & $\begin{array}{l}\text { A Systematic Review of the Integration of Motiva- } \\
\text { tional and Behavioural Theories in Game-based } \\
\text { Health Inter-ventions } \\
\text { Abdulsalam S. Mustafa, Nor'ashikin Ali, Jaspaljeet } \\
\text { Singh Dhillon }\end{array}$ \\
\hline Aidrina binti Mohamed Sofiadin \\
\hline 10:30 am - 10:45 am
\end{tabular}


Sessions Schedule

\begin{tabular}{|c|c|}
\hline & $\begin{array}{l}\text { Mira Afrina, Samsuryadi, Ab Razak Che Hussin and } \\
\text { Suraya Miskon }\end{array}$ \\
\hline $11: 30$ am - 11:45 am & $\begin{array}{l}\text { Analysis of Multimedia Elements Criteria using } \\
\text { AHP Method } \\
\text { Nadiah Mohamad Sofian, Ahmad Sobri Hashim, and } \\
\text { Aliza Sarlan }\end{array}$ \\
\hline $11: 45$ am - 12:00 pm & $\begin{array}{l}\text { The Development of a Criteria-Based Group For- } \\
\text { mation Systems for Student Group Work } \\
\text { Divya Gopal Mohan and Khairul Shafee Kalid }\end{array}$ \\
\hline & Parallel Session II \\
\hline Time & 1:00 pm - 3:45 pm (GMT time) \\
\hline Zoom link : & $\begin{array}{l}\text { https://us02web.zoom.us/j/86884421517?pwd } \\
\text { =aTNma1dCVmloSXZ0eVk20EdXYVpmQT09 }\end{array}$ \\
\hline Meeting ID : & 86884421517 \\
\hline Passcode : & IRICT2020 \\
\hline \multicolumn{2}{|c|}{ Main Room (Artificial Intelligence) } \\
\hline 1:00 pm - 1:15 pm & $\begin{array}{l}\text { Pulmonary Nodule Classification Based on Three } \\
\text { Convolutional Neural Networks Models } \\
\text { Enoumayri Elhoussaine and Belaqziz Salwa }\end{array}$ \\
\hline 1:15 pm - 1:30 pm & $\begin{array}{l}\text { Diagnosis of COVID-19 Disease Using Convolu- } \\
\text { tional Neural Network Models based Transfer } \\
\text { Learning } \\
\text { Hicham Moujahid, Bouchaib Cherradi, Mohammed Al- } \\
\text { Sarem and Lhoussain Bahatti }\end{array}$ \\
\hline $1: 30 \mathrm{pm}-1: 45 \mathrm{pm}$ & $\begin{array}{l}\text { Early Diagnoses of Parkinson's Using Dimension- } \\
\text { ality Reduction Techniques } \\
\text { Tariq Saeed Mian }\end{array}$ \\
\hline 1:45 pm - 2:00 pm & $\begin{array}{l}\text { A Long Short Term Memory and a Discrete } \\
\text { Wavelet Transform to Predict the Stock Price } \\
\text { Mu'tasem Jarrah and Naomie Salim }\end{array}$ \\
\hline
\end{tabular}


Sessions Schedule

\begin{tabular}{|c|c|}
\hline $2: 00 \mathrm{pm}-2: 15 \mathrm{pm}$ & $\begin{array}{l}\text { Optimized Text Classification using Correlated } \\
\text { Based Improved Genetic Algorithm } \\
\text { Thabit Sabbah }\end{array}$ \\
\hline $2: 15$ pm - 2:30 pm & $\begin{array}{l}\text { Multi-objective NPO Minimizing the Total Cost } \\
\text { and CO2 Emissions for a Stand-Alone Hybrid En- } \\
\text { ergy System } \\
\text { Abbas Q. Mohammed, Kassim A. Al-Anbarri, Rafid M. } \\
\text { Hannun }\end{array}$ \\
\hline & $\begin{array}{l}\text { A Real time Flood Detection System Based on Ma- } \\
\text { chine Learning Algorithms }\end{array}$ \\
\hline $2: 30 \mathrm{pm}-2: 45 \mathrm{pm}$ & $\begin{array}{l}\text { Abdirahman Osman Hashi, Abdullahi Ahmed } \\
\text { Abdirahman, Mohamed Abdirah-man Elmi, Siti Zaiton } \\
\text { Mohd Hashim }\end{array}$ \\
\hline $2: 45$ pm - 3:00 pm & $\begin{array}{l}\text { A Deep Neural Network Model with Multihop Self- } \\
\text { Attention mechanism for Topic Segmentation of } \\
\text { Texts } \\
\text { Fayçal Nouar and Hacene Belhadef }\end{array}$ \\
\hline 3:00 pm - 3:15 pm & $\begin{array}{l}\text { Comparative Study of SMOTE and Bootstrapping } \\
\text { Performance based on Predication Methods } \\
\text { Abdulaziz Aborujilah, Rasheed Mohammad Nassr, } \\
\text { Tawfik Al-Hadhrami, Mohd Nizam Husen, Nor Azlina } \\
\text { Ali, Abdulaleem Al- Othmani, Mustapha Hamdi }\end{array}$ \\
\hline $3: 15$ pm - 3:30 pm & $\begin{array}{l}\text { Convolutional Neural Networks for Automatic De- } \\
\text { tection of Colon Adenocarcinoma Based on Histo- } \\
\text { pathological Images } \\
\text { Yakoop Qasim, Habeb Al-Sameai, Osamah Ali and } \\
\text { Abdulelah Hassan }\end{array}$ \\
\hline $3: 30 \mathrm{pm}-3: 45 \mathrm{pm}$ & $\begin{array}{l}\text { A CNN-based Model for Early Melanoma Detection } \\
\text { Amer Sallam, Abdulfattah E. Ba Alawi, Ahmed Y. A. } \\
\text { Saeed }\end{array}$ \\
\hline
\end{tabular}


Sessions Schedule

\begin{tabular}{|c|c|}
\hline \multicolumn{2}{|c|}{ Room 1 (Data Science and Big Data Analytics) } \\
\hline 1:00 pm - 1:15 pm & $\begin{array}{l}\text { Big Data Analytics Model for Preventing the } \\
\text { Spread of COVID-19 During Hajj Using the Pro- } \\
\text { posed Smart Hajj Application } \\
\text { Ibtehal Nafea }\end{array}$ \\
\hline $1: 15$ pm - 1:30 pm & $\begin{array}{l}\text { A New Multi-Resource Deadlock Detection Algo- } \\
\text { rithm Using Directed Graph Requests in Distrib- } \\
\text { uted Database Systems } \\
\text { Khalid Al-Hussaini, Nabeel A. Al-Amdi and Fuaad } \\
\text { Hasan Abdulrazzak }\end{array}$ \\
\hline 1:30 pm - 1:45 pm & $\begin{array}{l}\text { Review on Emotion Recognition using EEG signals } \\
\text { Based on Brain-Computer Interface System } \\
\text { Mona Algarni and Faisal Saeed }\end{array}$ \\
\hline 1:45 pm - 2:00 pm & $\begin{array}{l}\text { Facial Recognition to identify Emotions: An appli- } \\
\text { cation of Deep Learning } \\
\text { Kenza Belhouchette }\end{array}$ \\
\hline $2: 00 \mathrm{pm}-2: 15 \mathrm{pm}$ & $\begin{array}{l}\text { Text-based Analysis to Detect Figure Plagiarism } \\
\text { Taiseer Abdalla Elfadil Eisa, Naomie Salim and Salha } \\
\text { Alzahrani }\end{array}$ \\
\hline $2: 15 \mathrm{pm}-2: 30 \mathrm{pm}$ & $\begin{array}{l}\text { A Virtual Exploration of Al-Masjid Al-Nabawi Us- } \\
\text { ing Leap Motion Controller } \\
\text { Slim Kammoun and Hamza Ghandorh }\end{array}$ \\
\hline $2: 30 \mathrm{pm}-2: 45 \mathrm{pm}$ & $\begin{array}{l}\text { A Fusion-Based Feature Selection Framework for } \\
\text { Microarray Data Classification } \\
\text { Talal Almutiri, Faisal Saeed, Manar Alassaf, Essa } \\
\text { Abdullah Hezzam }\end{array}$ \\
\hline 2:45 pm - 3:00 pm & $\begin{array}{l}\text { An Approach based Natural Language Processing } \\
\text { for DNA Sequences Encoding Using the Global Vec- } \\
\text { tors for Word Representation } \\
\text { Brahim Matougui, Hacene Belhadef and Ilham Kitouni }\end{array}$ \\
\hline
\end{tabular}


Sessions Schedule

\begin{tabular}{|c|c|}
\hline 3:00 pm - 3:15 pm & $\begin{array}{l}\text { A Comparative Study on Liver Tumor Detection } \\
\text { Using CT Images } \\
\text { Abdulfattah E. Ba Alawi, Ahmed Y. A. Saeed, Borhan } \\
\text { M. N. Radman, and Burhan T. Alzekri }\end{array}$ \\
\hline $3: 15$ pm - 3:30 pm & $\begin{array}{l}\text { Brain Tumor Diagnosis System Based on RM Im- } \\
\text { ages: A Comparative Study } \\
\text { Ahmed Y. A. Saeed, Abdulfattah E. Ba Alawi, Borhan } \\
\text { M. N. Radman }\end{array}$ \\
\hline $3: 30 \mathrm{pm}-3: 45 \mathrm{pm}$ & $\begin{array}{l}\text { Hospital Information System for Motivating Pa- } \\
\text { tient Loyalty: A Systematic Literature Review } \\
\text { Saleh Nasser Rashid Alismaili and Mohana } \\
\text { Shanmugam }\end{array}$ \\
\hline \multicolumn{2}{|r|}{ Room 2 (Information Security) } \\
\hline 1:00 pm - 1:15 pm & $\begin{array}{l}\text { Analysis and Evaluation of Template Based Meth- } \\
\text { ods against Geometric Attacks: A Survey } \\
\text { Tanya Koohpayeh Araghi, Ala Abdulsalam Alarood, } \\
\text { Sagheb Kohpayeh Araghi }\end{array}$ \\
\hline $1: 15 \mathrm{pm}-1: 30 \mathrm{pm}$ & $\begin{array}{l}\text { Survey of File Carving Techniques } \\
\text { Nor Ika Shahirah Ramli, Syifak Izhar Hisham, and } \\
\text { Mohd Faizal Abd Razak }\end{array}$ \\
\hline $1: 30 \mathrm{pm}-1: 45 \mathrm{pm}$ & $\begin{array}{l}\text { Affecting Factors in Information Security Policy } \\
\text { Compliance: Combine Organisational Factors and } \\
\text { User Habits } \\
\text { Angraini, Rose Alinda Alias and Okfalisa }\end{array}$ \\
\hline 1:45 pm - 2:00 pm & $\begin{array}{l}\text { An Efficient Fog-based Attack Detection Using En- } \\
\text { semble of MOA-WMA for Internet of Medical } \\
\text { Things } \\
\text { Shilan S. Hameed, Wan Haslina Hassan and Liza Abdul } \\
\text { Latiff }\end{array}$ \\
\hline $2: 00$ pm - 2:15 pm & $\begin{array}{l}\text { Security and Threats in The Internet of Things } \\
\text { Based Smart Home }\end{array}$ \\
\hline
\end{tabular}


Sessions Schedule

\begin{tabular}{|c|c|}
\hline & $\begin{array}{l}\text { Nor Fatimah Awang, Ahmad Fudhail Iyad Mohd } \\
\text { Zainudin, Syahaneim Marzuki , Syed Nasir Alsagoff, } \\
\text { Taniza Tajuddin, Ahmad Dahari Jarno }\end{array}$ \\
\hline 2:15 pm - 2:30 pm & $\begin{array}{l}\text { A New DNA Based Encryption Algorithm for Inter- } \\
\text { net of Things } \\
\text { Bassam Al-Shargabi and Mohammed Abbas Fadhil Al- } \\
\text { Husainy }\end{array}$ \\
\hline 2:30 pm - 2:45 pm & $\begin{array}{l}\text { Mitigation of Data Security Threats in Iraqi Dam } \\
\text { Management Systems: A Case Study of Fallujah } \\
\text { Dam Management System } \\
\text { Hussam J. Ali, Hiba Zuhair and Talib M. Jawad }\end{array}$ \\
\hline 2:45 pm - 3:00 pm & $\begin{array}{l}\text { An Advanced Encryption Cryptographically- } \\
\text { based Securing Applicative Protocols MQTT and } \\
\text { CoAP to Optimize Medical-IOT Supervising Plat- } \\
\text { forms } \\
\text { Sanaa EL AIDI, Abderrahim Bajit, Anass BARODI, } \\
\text { Habiba CHAOUI and Ahmed Tamtaoui }\end{array}$ \\
\hline 3:00 pm - 3:15 pm & $\begin{array}{l}\text { Anomaly Intrusion Detection Systems in IOT us- } \\
\text { ing Deep Learning Techniques: A Survey } \\
\text { Muaadh. A. Alsoufi, Shukor Razak, Maheyzah Md Siraj, } \\
\text { Abdulalem Ali, Maged Nasser, Salah Abdo }\end{array}$ \\
\hline $3: 15$ pm - 3:30 pm & $\begin{array}{l}\text { Robot Networks and Their Impact on Cyber Secu- } \\
\text { rity and Protection from Attacks: A Review } \\
\text { Daniah Anwar Hasan and Linah Faisal Tasji }\end{array}$ \\
\hline 3:30 pm - 3:45 pm & $\begin{array}{l}\text { Adopting React Personal Health Record (PHR) } \\
\text { System in Yemen HealthCare Institutions } \\
\text { Ziad Saif Alrobieh, Dhiaa Faisal Alshamy, Maged } \\
\text { Nasser }\end{array}$ \\
\hline
\end{tabular}


Sessions Schedule

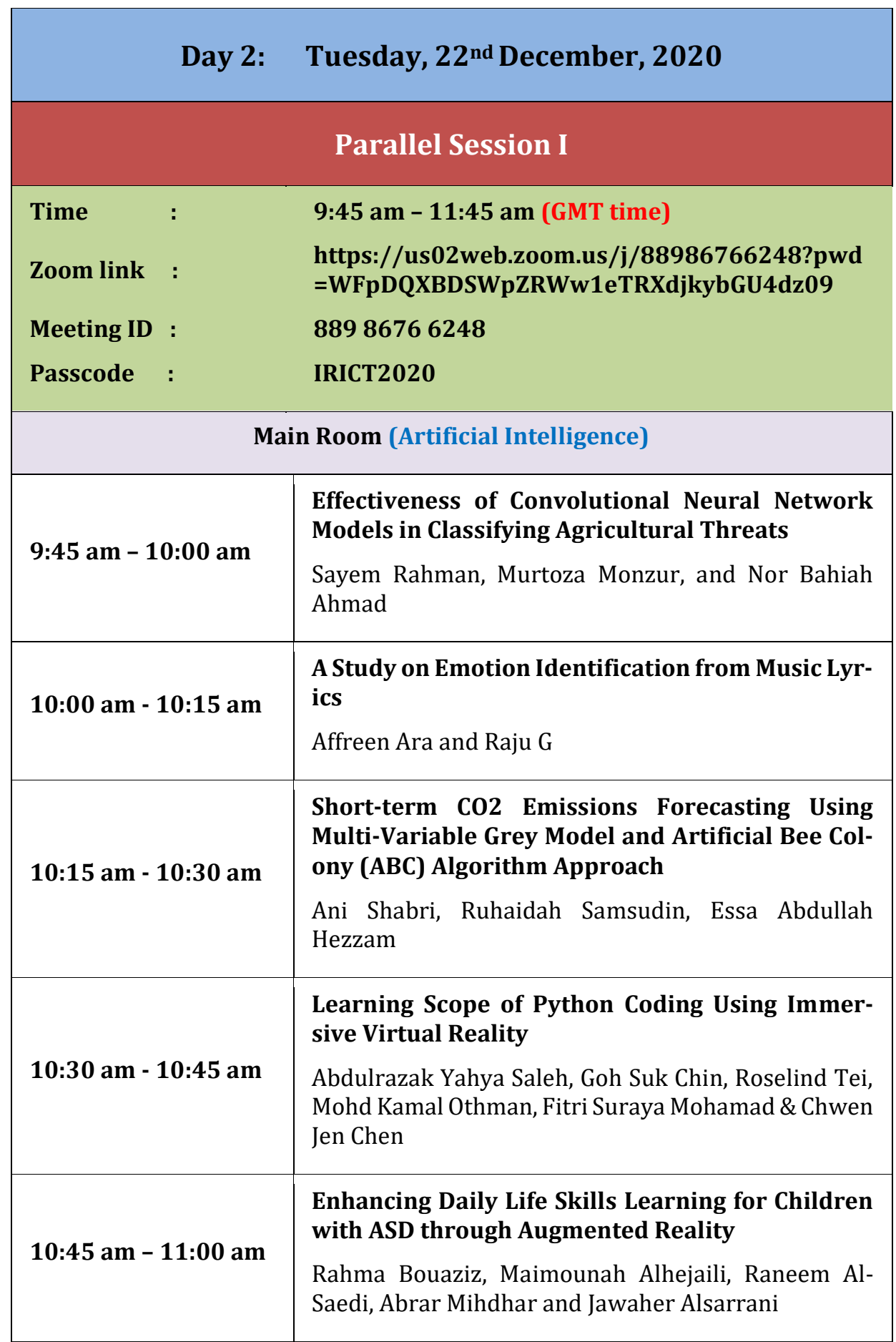


Sessions Schedule

\begin{tabular}{|c|c|}
\hline 11:00 am - 11:15 am & $\begin{array}{l}\text { Application of Shuffled Frog-Leaping Algorithm } \\
\text { for Optimal Software Project Scheduling and } \\
\text { Staffing } \\
\text { Ahmed O. Ameen, Hammed A. Mojeed, Abdulazeez T. } \\
\text { Bolariwa, } \\
\text { Abdullateef O. Balogun, Modinat A. Mabayoje, Fatima } \\
\text { E. Usman-Hamzah and Muyideen Abdulraheem }\end{array}$ \\
\hline 11:15 am - 11:30 am & $\begin{array}{l}\text { Watermarking Techniques for Mobile Applica- } \\
\text { tion: A Review } \\
\text { Aqilah Abd. Ghani, Syifak Izhar Hisham, Nur Alya } \\
\text { Afikah Usop, and Nor Bakiah Abd Warif }\end{array}$ \\
\hline 11:30 am - 11:45 am & $\begin{array}{l}\text { Context Ontology for Smart Healthcare Systems } \\
\text { Salisu Garba Radziah Mohamad and Nor Azizah } \\
\text { Saadon }\end{array}$ \\
\hline \multicolumn{2}{|c|}{ Room 1 (Data Science and Big Data Analytics) } \\
\hline 9:45 am - 10:00 am & $\begin{array}{l}\text { Comparison of Data Analytic Techniques for a } \\
\text { Spatial Opinion Mining in Literary Works: A Re- } \\
\text { view Paper } \\
\text { Sea Yun Ying, Pantea Keikhosrokiani and Moussa } \\
\text { Pourya Asl }\end{array}$ \\
\hline 10:00 am - 10:15 am & $\begin{array}{l}\text { The Digital Resources Objects Retrieval: Concepts } \\
\text { and Figures } \\
\text { Wafa' Za'al Alma'aitah, Abdullah Zawawi Talib, Mohd } \\
\text { Azam Osman }\end{array}$ \\
\hline 10:15 am - 10:30 am & $\begin{array}{l}\text { A Review of Graph-Based Extractive Text Summa- } \\
\text { rization Models } \\
\text { Abdulkadir Abubakar Bichi, Ruhaidah Samsudin, } \\
\text { Rohayanti Hassan and Khalil Almekhlafi }\end{array}$ \\
\hline
\end{tabular}


Sessions Schedule

\begin{tabular}{|c|c|}
\hline $10: 30$ am - 10:45 am & $\begin{array}{l}\text { Contrast Image Quality Assessment Algorithm } \\
\text { Based on Probability Density Functions Features } \\
\text { Ismail Taha Ahmed, Chen Soong Der, Norziana Jamil, } \\
\text { Baraa Tareq Hammad }\end{array}$ \\
\hline $10: 45$ am - 11:00 am & $\begin{array}{l}\text { The Impact of Data Augmentation on Accuracy of } \\
\text { COVID-19 Detection Based on X-ray Images. } \\
\text { Yakoop Qasim, Basheer Ahmed, Tawfeek Alhadad, } \\
\text { Habeb Al-Sameai and Osamah Ali }\end{array}$ \\
\hline 11:00 am - 11:15 am & $\begin{array}{l}\text { Smart Traffic Light System Design based on Single } \\
\text { Shot MultiBox Detector (SSD) and Anylogic Simu- } \\
\text { lation } \\
\text { E R Salim, A B Pantjawati, D Kuswardhana, A } \\
\text { Saripudin, N D Jayanto, Nurhi-dayatulloh, L A } \\
\text { Pratama }\end{array}$ \\
\hline 11:15 am - 11:30 am & $\begin{array}{l}\text { Automatic Audio Replacement of Objectionable } \\
\text { Content for Sri Lankan Locale } \\
\text { Gobiga Rajalingam, Janarthan Jeyachandran, M. S. M. } \\
\text { Siriwardane, Tharshvini Pathmaseelan, R.K.N.D. } \\
\text { Jayawardhane and N. S. Weerakoon }\end{array}$ \\
\hline 11:30 am - 11:45 am & $\begin{array}{l}\text { A Modified UTAUT Model for Hospital Infor- } \\
\text { mation Systems Geared Towards Motivating Pa- } \\
\text { tient Loyalty } \\
\begin{array}{l}\text { Saleh Nasser Rashid Alismaili and Mohana } \\
\text { Shanmugam }\end{array}\end{array}$ \\
\hline \multicolumn{2}{|r|}{ Room 2 (Information System) } \\
\hline 9:45 am - 10:00 am & $\begin{array}{l}\text { Potential Benefits of Social Media to Healthcare: A } \\
\text { Systematic Literature Review } \\
\text { Ghada Ahmad Abdelguiom and Noorminshah A.Iahad }\end{array}$ \\
\hline $10: 00$ am - 10:15 am & $\begin{array}{l}\text { Building Information Modelling Adoption: Sys- } \\
\text { tematic Literature Review } \\
\text { Hafiz Muhammad Faisal Shehzad, Roliana Binti } \\
\text { Ibrahim, Ahmad Fadhil Yusof, Khairul Anwar }\end{array}$ \\
\hline
\end{tabular}


Sessions Schedule

\begin{tabular}{|c|c|}
\hline & $\begin{array}{l}\text { Mohamed Khaidzir, Omayma Husain Abbas Hassan } \\
\text { and Samah Abdelsalam Abdalla }\end{array}$ \\
\hline $10: 15$ am - 10:30 am & $\begin{array}{l}\text { Adoption of Smart Cities Models in Developing } \\
\text { Countries: Focusing in Strategy and Design in Su- } \\
\text { dan } \\
\text { Mohmmed S. Adrees, Abdelrahman E. Karrar and } \\
\text { Waleed I. Osman }\end{array}$ \\
\hline $10: 30$ am - $10: 45$ am & $\begin{array}{l}\text { Student Compliance Intention Model for Contin- } \\
\text { ued Us-age of E-Learning in University } \\
\text { Ken Ditha Tania, Norris Syed Abdullah, Norasnita } \\
\text { Ahmad and Samsuryadi Sahmin }\end{array}$ \\
\hline $10: 45$ am - 11:00 am & $\begin{array}{l}\text { Digital Information and Communication Overload } \\
\text { among Youths in Malaysia: A Preliminary Review } \\
\text { Mohamad Ghozali Hassan, Muslim Diekola Akanmu, } \\
\text { Hussein Mohammed Esmail, Abualrejal, Amal } \\
\text { Abdulwahab Hasan Alamrani }\end{array}$ \\
\hline 11:00 am - 11:15 am & $\begin{array}{l}\text { The Effect of Using Social Networking Sites on Un- } \\
\text { dergraduate Students' Perception and Academic } \\
\text { Performance at University of Taiz - Yemen } \\
\text { Maged Rfeqallah, Rozilah Kasim Faisal A.M. Ali and } \\
\text { Yahya Abdul Ghaffar }\end{array}$ \\
\hline 11:15 am - 11:30 am & $\begin{array}{l}\text { Building Information Modelling Adoption Model } \\
\text { for Malaysian Architecture, Engineering and Con- } \\
\text { struction Industry } \\
\text { Hafiz Muhammad Faisal Shehzad, Roliana Binti } \\
\text { Ibrahim, Ahmad Fadhil Yusof, Khairul Anwar } \\
\text { Mohamed Khaidzir, Muhammad Mahboob Khurshid } \\
\text { and Farah Zeehan Othman }\end{array}$ \\
\hline $11: 30$ am - 11:45 am & $\begin{array}{l}\text { Digital Government Competency for Omani Public } \\
\text { Sector Managers: A Conceptual Framework } \\
\text { Juma Al-Mahrezi, Nur Azaliah Abu Bakar, and Nilam } \\
\text { Nur Amir Sjarif }\end{array}$ \\
\hline
\end{tabular}


Sessions Schedule

\section{Parallel Session II}

$\begin{array}{rll}\text { Time } & \text { 12:45 pm }-2: 45 \text { pm (GMT time) } \\ \text { Zoom link : } & \begin{array}{l}\text { https://us02web.zoom.us/j/88986766248?pwd } \\ \text { =WFpDQXBDSWpZRWw1eTRXdjkybGU4dz09 }\end{array} \\ \text { Meeting ID : } & 88986766248 \\ \text { Passcode } \quad: & \text { IRICT2020 }\end{array}$

Main Room (Computational Vision + Telecommunication)

\begin{tabular}{|l|l|}
\hline & Lossless Audio Steganographic Method Using
\end{tabular}

12:45 pm-1:00 pm Companding Technique

Ansam Osamah Abdulmajeed

A Comparison of CNN and Conventional Descriptors for Word Spotting Approach: Applica-

1:00 pm - 1:15 pm tion to Handwritten Document Image Retrieval

Ryma Benabdelaziz, Djamel Gaceb, Mohammed Haddad

Handwritten Arabic Characters Recognition: Comparison of Conventional Machine

1:15 pm - 1:30 pm Learning and Deep Learning Approaches

Faouci Soumia, Gaceb Djamel and Mohammed Haddad

Document Image Edge Detection Based on a Local Hysteresis Thresholding and Automatic Setting 1:30 pm - 1:45 pm Using PSO

Mohamed Benkhettou, Nibel Nadjeh, Djamel Gaceb

Fast I2SDBSCAN based on Integral Volume of 3D Histogram: Application to Color Layer Separation in Document Images

Zakia KEZZOULZA and Djamel GACEB

2:00 pm - 2:15 pm

Simulation and Control of Industrial Composition Process over Wired and Wireless Networks 
Sessions Schedule

\begin{tabular}{|c|c|}
\hline & $\begin{array}{l}\text { Hakim Qaid Abdullah Abdulrab, Fawnizu Azmadi } \\
\text { Hussin, Panneer Selvam Arun, Azlan Awang and Idris } \\
\text { Ismail }\end{array}$ \\
\hline $2: 15$ pm - 2:30 pm & $\begin{array}{l}\text { Design of Wireless Local Multimedia Communica- } \\
\text { tion Network (WLMmCN) based on Android Ap- } \\
\text { plication without Internet Connection } \\
\text { R. Q. Shaddad, F. A. Alqasemi, S. A. Alfaqih, M. F. } \\
\text { Alsabahi1, A. T. Fara1, K. M. Nejad, E. A. Albukhaiti }\end{array}$ \\
\hline $2: 30 \mathrm{pm}-2: 45 \mathrm{pm}$ & $\begin{array}{l}\text { A Statistical Channel Propagation Analysis for } 5 G \\
\text { mmWave at } 73 \mathrm{GHz} \text { in Urban Microcell } \\
\text { Zaid Ahmed Shamsan }\end{array}$ \\
\hline \multicolumn{2}{|r|}{ Room 1 (Software Engineering) } \\
\hline $12: 45$ pm - 1:00 pm & $\begin{array}{l}\text { SpaceScience App: Development of a Mobile Ap- } \\
\text { plication for School Children } \\
\text { Wan Fatimah Wan Ahmad and Ain Fatihah Ahmad } \\
\text { Harnaini }\end{array}$ \\
\hline 1:00 pm - 1:15 pm & $\begin{array}{l}\text { Research on Online Problem-Based Learning } \\
\text { Among Undergraduate Students: A Systematic Re- } \\
\text { view } \\
\text { Amira Saif and Irfan Umar }\end{array}$ \\
\hline 1:15 pm - 1:30 pm & $\begin{array}{l}\text { Teamwork Communication in Healthcare: An In- } \\
\text { strument (questionnaire) Validation Process } \\
\text { Wasef Matar and Monther Aldwair }\end{array}$ \\
\hline 1:30 pm - 1:45 pm & $\begin{array}{l}\text { Measuring Risk Mitigation Techniques in Agile } \\
\text { Global Software Development } \\
\text { Adila Firdaus Arbain, Muhammad Akil Rafeek, } \\
\text { Zuriyaninatasa Podari and Cik Feresa Mohd Foozy }\end{array}$ \\
\hline 1:45 pm - 2:00 pm & $\begin{array}{l}\text { Risk Mitigation Framework for Agile Global Soft- } \\
\text { ware Development } \\
\text { Zuriyaninatasa Podari, Adila Firdaus Arbain, Noraini } \\
\text { Ibrahim1 and Endah Surdamilah }\end{array}$ \\
\hline
\end{tabular}




\section{Sessions Schedule}

\begin{tabular}{|c|c|}
\hline 2:00 pm - 2:15 pm & $\begin{array}{l}\text { Re-Verification of the Improved Software Project } \\
\text { Monitoring Task Model of the Agile Kanban (i- } \\
\text { KAM) } \\
\text { Hamzah Alaidaros, Mazni Omar, Rohaida Romli, } \\
\text { Adnan Hussein }\end{array}$ \\
\hline $2: 15$ pm - 2:30 pm & $\begin{array}{l}\text { Factors Affecting Customer Acceptance of Online } \\
\text { Shopping Platforms in Malaysia: Conceptual } \\
\text { model and Preliminary Results } \\
\text { Nabil Hasan Al-kumaim, Gan Wong Sow, Fathey } \\
\text { Mohammed }\end{array}$ \\
\hline $2: 30 \mathrm{pm}-2: 45 \mathrm{pm}$ & $\begin{array}{l}\text { Multi-Domain Business Intelligence Model for Ed- } \\
\text { ucational Enterprise Resource Planning Systems } \\
\text { Hisham Abdullah, Azman Taa and Fathey Mohammed }\end{array}$ \\
\hline \multicolumn{2}{|c|}{ Room 2 (Telecommunication and IoT) } \\
\hline $12: 45 \mathrm{pm}-1: 00 \mathrm{pm}$ & $\begin{array}{l}\text { A Reliable Single Prediction Data Reduction Ap- } \\
\text { proach for WSNs based on Kalman Filter } \\
\text { Zaid Yemeni, Haibin Wang, Waleed M.Ismael, Younis } \\
\text { Ibrahim, and Peng Li }\end{array}$ \\
\hline $1: 00 \mathrm{pm}-1: 15 \mathrm{pm}$ & $\begin{array}{l}\text { A Real-Time Groundwater Level Monitoring Sys- } \\
\text { tem Based on WSN, Taiz, Yemen } \\
\text { Asma'a K. Akershi, Ziad S. Arobieh, Reayidh A. Ahmed }\end{array}$ \\
\hline 1:15 pm - 1:30 pm & $\begin{array}{l}\text { Design and Simulation of Multiband Circular Mi- } \\
\text { crostrip Patch Antenna with CSRR for WLAN and } \\
\text { WiMAX Ap-plications } \\
\text { Abdulguddoos S. A. Gaid, Amer A. Sallam, Mohamed H. } \\
\text { M. Qasem, Maged S. G. Abbas and Amjad M. H. Aoun }\end{array}$ \\
\hline $1: 30$ pm - 1:45 pm & $\begin{array}{l}\text { Reference Architectures for the IoT: A Survey } \\
\text { Raghdah Saemaldahr, Bijayita Thapa, Kristopher } \\
\text { Maikoo, and Eduardo B. Fernandez }\end{array}$ \\
\hline
\end{tabular}


Sessions Schedule

\begin{tabular}{|c|c|}
\hline 1:45 pm - 2:00 pm & $\begin{array}{l}\text { A Circular Multiband Microstrip Patch Antenna } \\
\text { with } \\
\text { WLAN/WiMAX/Bluetooth/UMTS/LTE } \\
\text { Abdulguddoos S. A. Gaid, Amer A. Sallam, Mohamed H. } \\
\text { M. Qasem, Maged S. G. Abbas, and Amjad M. H. Aoun }\end{array}$ \\
\hline $2: 00 \mathrm{pm}-2: 15 \mathrm{pm}$ & $\begin{array}{l}\text { Compact Wide-Bandwidth Microstrip Antenna for } \\
\text { Millimeter Wave Applications } \\
\text { Osaid Abdulrahman Saeed, Moheeb Ali Ameer, } \\
\text { Mansour Noman Ghaleb }\end{array}$ \\
\hline $2: 15$ pm - 2:30 pm & $\begin{array}{l}\text { Dual-Band Rectangular Microstrip Patch Antenna } \\
\text { with CSRR for 28/38 GHz Bands Applications } \\
\text { Abdulguddoos S. A. Gaid, Mohamed H. M. Qasem, } \\
\text { Amer A. Sallam and Ebrahim Q. M. Shayea }\end{array}$ \\
\hline $2: 30 \mathrm{pm}-2: 45 \mathrm{pm}$ & $\begin{array}{l}\text { Dual Band Rectangular Microstrip Patch Antenna } \\
\text { for 5G Millimeter-Wave Wireless Access and } \\
\text { Backhaul Applications } \\
\text { Abdulguddoos S. A. Gaid, Amer A. Sallam, Amjad M. H. } \\
\text { Aoun, Ahmed A. A. Saeed, and Osama Y. A. Sae'ed }\end{array}$ \\
\hline
\end{tabular}


Table of Contents

Organizing Committee ii

Technical Committee iv

Messages vii

Keynote Speakers $\quad$ xii

Conference Program xviii

Session Schedule $\quad$ xxii

Intelligent Health Informatics 1

Comparative Study of SMOTE and Bootstrapping Perfor- 2 mance based on Predication Methods

Abdulaziz Aborujilah, Rasheed Mohammad Nassr, Tawfik Al-Hadhrami, Mohd Nizam Husen, Nor Azlina Ali, Abdulaleem Al- Othmani, Mustapha Hamdi

UPLX: Blockchain Platform for Integrated Health Data Management

Omar Musa1, Lim Shu Yun, Reza Ismail

Convolutional Neural Networks for Automatic Detection of Colon Adenocarcinoma Based on Histopathological Images

Yakoop Qasim, Habeb Al-Sameai, Osamah Ali and Abdulelah Hassan

Intelligent Health Informatics with Personalisation in Weather-based Healthcare using Machine Learning

Radiah Haque, Sin-Ban Ho, Ian Chai, Chin-Wei Teoh1, Adina Abdullah, Chuie-Hong Tan, and Khairi Shazwan Dollmat

A CNN-based Model for Early Melanoma Detection

Amer Sallam, Abdulfattah E. Ba Alawi, Ahmed Y. A. Saeed

SMARTS D4D Application Module for Dietary Adherence Self-Monitoring among Hemodialysis Patients

Hafzan Yusoff Nur Intan Raihana Ruhaiyem and Mohd Hakim Zakaria 
Improved Multi-Label Medical Text Classification using Features Cooperation

Rim Chaib, Nabiha Azizi, Nawel Zemmal, Didier Schwab and Samir Brahim Belhaouari

Image Modeling through Augmented Reality for Skin Allergies Recognition

Nur Intan Raihana Ruhaiyem and Nur Amalina Mazlan

Hybridisation of Optimised Support Vector Machine and Artificial Neural Network for Diabetic Retinopathy Classification

Nur Izzati Ab Kader, Umi Kalsom Yusof and Maziani Sabudin

A Habit-Change Support Web-Based System with Big Data Analytical Features for Hospitals (Doctive)

Cheryll Anne Augustine and Pantea Keikhosrokiani

An Architecture for Intelligent Diagnosing Diabetic Types and Complications Based on Symptoms

Gunasekar Thangarasu, P.D.D. Dominic and Kayalvizhi Subramanian

An Advanced Encryption Cryptographically-based Securing Applicative Protocols MQTT and CoAP to Optimize MedicalIOT Supervising Platforms

Sanaa EL AIDI, Abderrahim Bajit, Anass BARODI, Habiba CHAOUI and Ahmed Tamtaoui

Pulmonary Nodule Classification Based on Three Convolutional Neural Networks Models

Enoumayri Elhoussaine and Belaqziz Salwa

A Comparative Study on Liver Tumor Detection Using CT Images

Abdulfattah E. Ba Alawi, Ahmed Y. A. Saeed, Borhan M. N. Radman, and Burhan T. Alzekri

Brain Tumor Diagnosis System Based on RM Images: A Comparative Study 
Ahmed Y. A. Saeed, Abdulfattah E. Ba Alawi, Borhan M. N. Radman

Diagnosis of COVID-19 Disease Using Convolutional Neural Network Models based Transfer Learning

Hicham Moujahid, Bouchaib Cherradi, Mohammed Al-Sarem and Lhoussain Bahatti

Early Diagnosos of Parkinson's Using Dimensionality Reduction Techniques

Tariq Saeed Mian

Detection of Cardiovascular Disease using Ensemble Machine Learning Techniques

Fizza Kashif and Umi Kalsom Yusof

\section{Health Information Management}

Hospital Information System for Motivating Patient Loyalty: A Systematic Literature Review

Saleh Nasser Rashid Alismaili and Mohana Shanmugam

Context Ontology for Smart Healthcare Systems

Salisu Garba Radziah Mohamad and Nor Azizah Saadon

A Modified UTAUT Model for Hospital Information Systems Geared Towards Motivating Patient Loyalty

Saleh Nasser Rashid Alismaili and Mohana Shanmugam

Teamwork Communication in Healthcare: An Instrument (questionnaire) Validation Process

Wasef Matar and Monther Aldwair

Potential Benefits of Social Media to Healthcare: A Systematic Literature Review

Ghada Ahmad Abdelguiom and Noorminshah A.Iahad

Exploring the Influence of Human-centered Design on User 26 Experience in Health Informatics Sector: A Systematic Review Lina Fatini Azmi and Norasnita Ahmad 
An Emotional-Persuasive Habit-Change Support Mobile Application for Heart Disease Patients (BeHabit)

Bhavani Devi Ravichandran and Pantea Keikhosrokiani

A Systematic Review of the Integration of Motivational and Behavioural Theories in Game-based Health Interventions

Abdulsalam S. Mustafa, Nor'ashikin Ali, Jaspaljeet Singh Dhillon

Adopting React Personal Health Record (PHR) System in Yemen HealthCare Institutions

Ziad Saif Alrobieh, Dhiaa Faisal Alshamy, Maged Nasser

\section{Artificial Intelligence and Soft Computing}

Application of Shuffled Frog-Leaping Algorithm for Optimal Software Project Scheduling and Staffing

Ahmed O. Ameen, Hammed A. Mojeed, Abdulazeez T.

Bolariwa,

Abdullateef O. Balogun, Modinat A. Mabayoje, Fatima E.

Usman-Hamzah and Muyideen Abdulraheem

A Long Short Term Memory and a Discrete Wavelet Transform to Predict the Stock Price

Mu'tasem Jarrah and Naomie Salim

Effective Web Service Classification Using a Hybrid of Ontology Generation and Machine Learning Algorithm

Murtoza Monzur, Radziah Mohamad and Nor Azizah Saadon

Binary Cuckoo Optimisation Algorithm and Information Theory for Filter-Based Feature Selection

Ali Muhammad Usman, Umi Kalsom Yusof and Syibrah Naim

Optimized Text Classification using Correlated Based Improved Genetic Algorithm

Thabit Sabbah

Multi-objective NPO Minimizing the Total Cost and $\mathrm{CO} 2$ Emissions for a Stand-Alone Hybrid Energy System

Abbas Q. Mohammed, Kassim A. Al-Anbarri, Rafid M. Hannun 
A Real time Flood Detection System Based on Machine Learning Algorithms

Abdirahman Osman Hashi, Abdullahi Ahmed Abdirahman, Mohamed Abdirah-man Elmi, Siti Zaiton Mohd Hashim

Extracting Semantic Concepts and Relations from Scientific Publications by Using Deep Learning

Fatima N. AL-Aswadi, Huah Yong Chan and Keng Hoon Gan

Effectiveness of Convolutional Neural Network Models in Classifying Agricultural Threats

Sayem Rahman, Murtoza Monzur, and Nor Bahiah Ahmad

A Study on Emotion Identification from Music Lyrics

Affreen Ara and Raju G

A Deep Neural Network Model with Multihop Self-Attention mechanism for Topic Segmentation of Texts

Fayçal Nouar and Hacene Belhadef

\section{Data Science and Big Data Analytics}

Big Data Interoperability Framework for Malaysian Public Open Data

Najhan Muhamad Ibrahim, Amir Aatieff Amir Hussin, Khairul Azmi Hassan and Ciara Breathnach

The Digital Resources Objects Retrieval: Concepts and Figures

Wafa' Za'al Alma'aitah, Abdullah Zawawi Talib, Mohd Azam Osman

A Review of Graph-Based Extractive Text Summarization Models

Abdulkadir Abubakar Bichi, Ruhaidah Samsudin, Rohayanti Hassan and Khalil Almekhlafi

Review on Emotion Recognition using EEG signals Based on Brain-Computer Interface System

Mona Algarni and Faisal Saeed 
A New Multi-Resource Deadlock Detection Algorithm Using Directed Graph Requests in Distributed Database Systems

Khalid Al-Hussaini, Nabeel A. Al-Amdi and Fuaad Hasan Abdulrazzak

Big Data Analytics Model for Preventing the Spread of COVID-19 During Hajj Using the Proposed Smart Hajj Application

Ibtehal Nafea

Financial Time Series Forecasting Using Prophet

Umi Kalsom Yusof, Mohd Nor Akmal Khalid, Abir Hussain and Haziqah Shamsudin

Facial Recognition to identify Emotions: An application of Deep Learning

Kenza Belhouchette

Text-based Analysis to Detect Figure Plagiarism

Taiseer Abdalla Elfadil Eisa, Naomie Salim and Salha Alzahrani

A Virtual Exploration of Al-Masjid Al-Nabawi Using Leap Motion Controller

Slim Kammoun and Hamza Ghandorh

Comparison of Data Analytic Techniques for a Spatial Opinion Mining in Literary Works: A Review Paper

Sea Yun Ying, Pantea Keikhosrokiani and Moussa Pourya Asl

Open Data in Prediction using Machine Learning: A Systematic Review

Norismiza Ismail and Umi Kalsom Yusof

Big Data Analytics Based Model for Red Chili Agirculture in Indonesia

Junita Juwita Siregar and Arif Imam Suroso

A Fusion-Based Feature Selection Framework for Microarray Data Classification 
Talal Almutiri, Faisal Saeed, Manar Alassaf, Essa Abdullah Hezzam

An Approach based Natural Language Processing for DNA Sequences Encoding Using the Global Vectors for Word Representation

Brahim Matougui, Hacene Belhadef and Ilham Kitouni

Short-term CO2 Emissions Forecasting Using Multi-Variable Grey Model and Artificial Bee Colony (ABC) Algorithm Approach

Ani Shabri, Ruhaidah Samsudin, Essa Abdullah Hezzam

\section{IoT and Intelligent Communication Systems}

A Reliable Single Prediction Data Reduction Ap-proach for WSNs based on Kalman Filter

Zaid Yemeni, Haibin Wang, Waleed M.Ismael, Younis Ibrahim, and Peng Li

A Real-Time Groundwater Level Monitoring System Based on WSN, Taiz, Yemen

Asma'a K. Akershi, Ziad S. Arobieh, Reayidh A. Ahmed

Design and Simulation of Multiband Circular Microstrip Patch Antenna with CSRR for WLAN and WiMAX Ap-plications

Abdulguddoos S. A. Gaid, Amer A. Sallam, Mohamed H. M. Qasem, Maged S. G. Abbas and Amjad M. H. Aoun

Reference Architectures for the IoT: A Survey

Raghdah Saemaldahr, Bijayita Thapa, Kristopher Maikoo, and Eduardo B. Fernandez

A Circular Multiband Microstrip Patch Antenna with DGS for WLAN/WiMAX/Bluetooth/UMTS/LTE

Abdulguddoos S. A. Gaid, Amer A. Sallam, Mohamed H. M. Qasem, Maged S. G. Abbas, and Amjad M. H. Aoun 
Anomaly Intrusion Detection Systems in IOT using Deep Learning Techniques: A Survey

Muaadh. A. Alsoufi, Shukor Razak, Maheyzah Md Siraj, Abdulalem Ali, Maged Nasser, Salah Abdo

Security and Threats in The Internet of Things Based Smart 66 Home

Nor Fatimah Awang, Ahmad Fudhail Iyad Mohd Zainudin, Syahaneim Marzuki , Syed Nasir Alsagoff, Taniza Tajuddin, Ahmad Dahari Jarno

Simulation and Control of Industrial Composition Process 67 over Wired and Wireless Networks

Hakim Qaid Abdullah Abdulrab, Fawnizu Azmadi Hussin, Panneer Selvam Arun, Azlan Awang and Idris Ismail

Performance Degradation of Multi-class Classification Model due to Continuous Evolving Data Streams

Abdul Sattar Palli, Jafreezal Jaafar, Manzoor Ahmed Hashmani

Compact Wide-Bandwidth Microstrip Antenna for Millimeter Wave Applications

Osaid Abdulrahman Saeed, Moheeb Ali Ameer, Mansour Noman Ghaleb

Dual-Band Rectangular Microstrip Patch Antenna with CSRR for 28/38 GHz Bands Applications

Abdulguddoos S. A. Gaid, Mohamed H. M. Qasem, Amer A. Sallam and Ebrahim Q. M. Shayea

Dual Band Rectangular Microstrip Patch Antenna for 5G Millimeter-Wave Wireless Access and Backhaul Applications

Abdulguddoos S. A. Gaid, Amer A. Sallam, Amjad M. H. Aoun, Ahmed A. A. Saeed, and Osama Y. A. Sae'ed

Design of Wireless Local Multimedia Communication Network (WLMmCN) based on Android Application without Internet Connection 
R. Q. Shaddad, F. A. Alqasemi, S. A. Alfaqih, M. F. Alsabahi1,

A. T. Fara1, K. M. Nejad, E. A. Albukhaiti

A Statistical Channel Propagation Analysis for 5G mmWave at $73 \mathrm{GHz}$ in Urban Microcell

Zaid Ahmed Shamsan

\section{Advances in Information Security}

Robot Networks and Their Impact on Cyber Security and Protection from Attacks: A Review

Daniah Anwar Hasan and Linah Faisal Tasji

An Efficient Fog-based Attack Detection Using Ensemble of MOA-WMA for Internet of Medical Things

Shilan S. Hameed, Wan Haslina Hassan and Liza Abdul Latiff

A New DNA Based Encryption Algorithm for Internet of Things

Bassam Al-Shargabi and Mohammed Abbas Fadhil Al-Husainy

Watermarking Techniques for Mobile Application: A Review

Aqilah Abd. Ghani, Syifak Izhar Hisham, Nur Alya Afikah Usop, and Nor Bakiah Abd Warif

Analysis and Evaluation of Template Based Methods against

Geometric Attacks: A Survey

Tanya Koohpayeh Araghi, Ala Abdulsalam Alarood, Sagheb Kohpayeh Araghi

Survey of File Carving Techniques

80

Nor Ika Shahirah Ramli, Syifak Izhar Hisham, and Mohd Faizal Abd Razak

Affecting Factors in Information Security Policy Compliance: Combine Organisational Factors and User Habits

Angraini, Rose Alinda Alias and Okfalisa 
Mitigation of Data Security Threats in Iraqi Dam Management Systems: A Case Study of Fallujah Dam Management System

Hussam J. Ali, Hiba Zuhair and Talib M. Jawad

\section{Advances in Information Systems}

Development and Validation of a Classified Information Assurance Scale for Institutions of Higher Learning

Bello Ahmadu, Ab Razak Che Hussin and Mahadi Bahari

Sustainable e-Learning Framework: Expert Views

Aidrina binti Mohamed Sofiadin

Derivation of a Customer Loyalty Factors Based on Customers' Changing Habits in E-Commerce Platform

Mira Afrina, Samsuryadi, Ab Razak Che Hussin and Suraya Miskon

Analysis of Multimedia Elements Criteria using AHP Method

Nadiah Mohamad Sofian, Ahmad Sobri Hashim, and Aliza Sarlan

The Development of a Criteria-Based Group Formation Systems for Student Group Work

Divya Gopal Mohan and Khairul Shafee Kalid

Trusted Factors of Social Commerce Product Review Video

Humaira Hairudin Halina Mohamed Dahlan Ahmad Fadhil Yusof

Building Information Modelling Adoption: Systematic Literature Review

Hafiz Muhammad Faisal Shehzad, Roliana Binti Ibrahim, Ahmad Fadhil Yusof, Khairul Anwar Mohamed Khaidzir, Omayma Husain Abbas Hassan and Samah Abdelsalam Abdalla

Adoption of Smart Cities Models in Developing Countries: 
Mohmmed S. Adrees, Abdelrahman E. Karrar and Waleed I. Osman

Factors Affecting Customer Acceptance of Online Shopping Platforms in Malaysia: Conceptual model and Preliminary Results

Nabil Hasan Al-kumaim, Gan Wong Sow, Fathey Mohammed

Student Compliance Intention Model for Continued Us-age of E-Learning in University

Ken Ditha Tania, Norris Syed Abdullah, Norasnita Ahmad and Samsuryadi Sahmin

Digital Information and Communication Overload among Youths in Malaysia: A Preliminary Review

Mohamad Ghozali Hassan, Muslim Diekola Akanmu, Hussein Mohammed Esmail, Abualrejal, Amal Abdulwahab Hasan Alamrani

The Effect of Using Social Networking Sites on Undergraduate Students' Perception and Academic Performance at University of Taiz - Yemen

Maged Rfeqallah, Rozilah Kasim Faisal A.M. Ali and Yahya Abdul Ghaffar

Building Information Modelling Adoption Model for Malaysian Architecture, Engineering and Construction Industry

Hafiz Muhammad Faisal Shehzad, Roliana Binti Ibrahim, Ahmad Fadhil Yusof, Khairul Anwar Mohamed Khaidzir, Muhammad Mahboob Khurshid and Farah Zeehan Othman

Digital Government Competency for Omani Public Sector Managers: A Conceptual Framework

Juma Al-Mahrezi, Nur Azaliah Abu Bakar, and Nilam Nur Amir Sjarif

\section{Computational Vision and Robotics}


Landmark Localization in Occluded Faces Using Deep Learning Approach

Zieb Rabie Alqahtani, Mohd Shahrizal Sunar, Abdulaziz A Alashbi

Contrast Image Quality Assessment Algorithm Based on Probability Density Functions Features

Ismail Taha Ahmed, Chen Soong Der, Norziana Jamil, Baraa Tareq Hammad

The Impact of Data Augmentation on Accuracy of COVID-19 Detection Based on X-ray Images.

Yakoop Qasim, Basheer Ahmed, Tawfeek Alhadad, Habeb AlSameai and Osamah Ali

A Fusion Schema of Hand-Crafted Feature and Feature Learning for Kinship Verification

Mohammed Ali Almuashi, Siti Zaiton Mohd Hashim, Nooraini Yusoff, Khairul Nizar Syazwan

Lossless Audio Steganographic Method Using Companding Technique

Ansam Osamah Abdulmajeed

Smart Traffic Light System Design based on Single Shot MultiBox Detector (SSD) and Anylogic Simulation

E R Salim, A B Pantjawati, D Kuswardhana, A Saripudin, N D Jayanto, Nurhi-dayatulloh, L A Pratama

Learning Scope of Python Coding Using Immersive Virtual Reality

Abdulrazak Yahya Saleh, Goh Suk Chin, Roselind Tei, Mohd Kamal Othman, Fitri Suraya Mohamad \& Chwen Jen Chen

Automatic Audio Replacement of Objectionable Content for Sri Lankan Locale

Gobiga Rajalingam, Janarthan Jeyachandran, M. S. M. Siriwardane, Tharshvini Pathmaseelan, R.K.N.D. Jayawardhane and N. S. Weerakoon 
A Comparison of CNN and Conventional Descriptors for Word Spotting Approach: Application to Handwritten Document Image Retrieval

Ryma Benabdelaziz, Djamel Gaceb, Mohammed Haddad

Handwritten Arabic Characters Recognition: Comparison of Conventional Machine Learning and Deep Learning Approaches

Faouci Soumia, Gaceb Djamel and Mohammed Haddad

Document Image Edge Detection Based on a Local Hysteresis Thresholding and Automatic Setting Using PSO

Mohamed Benkhettou, Nibel Nadjeh, Djamel Gaceb

Fast I2SDBSCAN based on Integral Volume of 3D Histogram: Application to Color Layer Separation in Document Images

Zakia KEZZOULZA and Djamel GACEB

Enhancing Daily Life Skills Learning for Children with ASD through Augmented Reality

Rahma Bouaziz, Maimounah Alhejaili, Raneem Al-Saedi, Abrar Mihdhar and Jawaher Alsarrani

\section{Recent Computing and Software Engineering}

SpaceScience App: Development of a Mobile Application for School Children

Wan Fatimah Wan Ahmad and Ain Fatihah Ahmad Harnaini

Research on Online Problem-Based Learning Among Undergraduate Students: A Systematic Review

Amira Saif and Irfan Umar

Derivation of Factors in Dealing Negative E-WOM for Maintaining Online Reputation

Rizka Dhini Kurnia, Halina Mohamed Dahlan, Samsuryadi 
A Terms Interrelationship Approach to Query Expansion Based on Terms Selection

Nuhu Yusuf, Mohd Amin Mohd Yunus, Norfaradilla Wahid, Mohd Najib Mohd Saleh, and Aida Mustapha

Multi-Domain Business Intelligence Model for Educational Enterprise Resource Planning Systems

Hisham Abdullah, Azman Taa and Fathey Mohammed

Measuring Risk Mitigation Techniques in Agile Global Software Development

Adila Firdaus Arbain, Muhammad Akil Rafeek, Zuriyaninatasa Podari and Cik Feresa Mohd Foozy

Risk Mitigation Framework for Agile Global Software Development

Zuriyaninatasa Podari, Adila Firdaus Arbain, Noraini Ibrahim1 and Endah Surdamilah

Re-Verification of the Improved Software Project Monitoring Task Model of the Agile Kanban (i-KAM)

Hamzah Alaidaros, Mazni Omar, Rohaida Romli, Adnan Hussein 
ABSTRACTS

Intelligent Health Informatics 
ABSTRACTS

\title{
Comparative study of SMOTE and Bootstrapping Performance based on Predication methods
}

\author{
Abdulaziz Aborujilah ${ }^{1}$, Rasheed Mohammad Nassr ${ }^{1}$, Tawfik Al-Hadhrami ${ }^{2}$, Mohd \\ Nizam Husen ${ }^{1}$, Nor Azlina Ali ${ }^{1}$, Abdulaleem Al- Othmani ${ }^{1}$, Mustapha Hamdi ${ }^{3}$ \\ ${ }^{1}$ University Kuala Lumpur, 50250 Kuala Lumpur , Malaysia \\ ${ }^{2}$ Nottingham Trent University, Nottingham NG1 4FQ, UK \\ ${ }^{3}$ Edge IA, IoT \\ Abdulazizsaleh@unikl.edu.my
}

\begin{abstract}
Recently, there has been a renewed interest in smart health systems that aim to deliver high quality healthcare services. Prediction methods are very essential to support these systems. They mainly rely on datasets with assumptions that match the reality. However, one of the greatest challenges to prediction methods is to have datasets which are normally distributed. This paper presents an experimental work to implement SMOTE (Synthetic Minority Oversampling Technique) and bootstrapping methods to normalize datasets. It also measured the impact of both methods in the performance of different prediction methods such as Support vector machine (SVM), Naive Bayes, and neural network(NN) The results showed that bootstrapping with native bays yielded better prediction performance as compared to other prediction methods with SMOTE.
\end{abstract}

Keywords: Datasets Normalization, Prediction Systems, Dataset redistribution Methods, SMOTE-Bootstrapping 
ABSTRACTS

\title{
UPLX: Blockchain Platform for Integrated Health Data Management
}

\author{
Omar Musa ${ }^{1}$, Lim Shu Yun ${ }^{1}$, Reza Ismail ${ }^{2}$ \\ ${ }^{1}$ Faculty of Business and Technology, UNITAR International University, 47300 Petaling Jaya, \\ Malaysia \\ ${ }^{2}$ LedgerX International Sdn Bhd, T3-20-3A Icon City Trade Center, 47300 Petaling Jaya, Ma- \\ laysia \\ \{omarm,lim_sy\}@unitar.my, reza@ledgerx.io
}

\begin{abstract}
Health data management currently needs a technology refresh in order to provide accurate, reliable and verifiable data for doctors and researchers to decide on the best medications and for the public to have their own dependable health information history as they continue with their daily lives. We propose UPLX (Unified Patient Ledger) as a blockchain-based data platform to securely record, "anonymize" and store patient health data for medical, academic and pharmaceutical research. UPLX is a blockchain powered health data platform which is designed to be interoperable and can be integrated with any hospital information systems (HIS) through API (Application Programming Interface) technology. To this end, a Hyperledger Fabric implementation is described to demonstrate the feasibility of the proposal and its use in healthcare organization. Successful implementation will accelerate the acceptance of Blockchain technology in protecting recorded health data while increasing the efficiency of healthcare delivery.
\end{abstract}

Keywords: Blockchain, Interoperable, Medical Ledger, Hyperledger Fabric 
ABSTRACTS

\title{
Convolutional Neural Networks for Automatic Detection of Colon Adenocarcinoma Based on Histopathological Images.
}

\author{
Yakoop Qasim, Habeb Al-Sameai, Osamah Ali and Abdulelah Hassan \\ Department of Mechatronics and Robotics Engineering, Taiz University, Yemen. \\ yakoopra@gmail.com, habeebabdu.alkhaliq1230@gmail.com, \\ osamahfarhan1995@gmail.com, abdmoh98710@gmail.com
}

\begin{abstract}
Colorectal cancer is the second type of cancer that causes death and the third in terms of prevalence and number of cases. Due to the absence of symptoms in the early stages of the injury, several types of tests must be performed to discover the cancer, but these methods take a lot of time, cost and require a specialized expert. So in this paper, we proposed a Convolutional Neural Network (CNN) model that characterized by speed of diagnosis and high accuracy with few number of parameters for diagnosing colon adenocarcinoma since it is the most common of colorectal cancer, where it represents $95 \%$ of the total cases of colorectal cancer, depending on dataset of 10000 histopathological images divided into 5000 images for colon adenocarcinoma and 5000 images for benign colon. Our model consists of two paths each path is responsible for creating 256 feature maps to increase the number of features at different level in order to improve the accuracy and sensitivity of the classification. To compare the performance of the proposed model, Visual geometry Group (VGG16) model was prepared and trained on the same dataset. After training the two models we obtained an accuracy of $99.6 \%, 96.2 \%$ for the proposed model and VGG16 respectively, we also obtained from the proposed model a sensitivity of $99.6 \%$ and Area Under Curve (AUC) of $99.6 \%$ which indicates the effectiveness of this model in diagnosing colon adenocarcinoma.
\end{abstract}

Keywords: Deep learning, Colorectal Cancer, Convolutional Neural Networks. 
ABSTRACTS

\title{
Intelligent Health Informatics with Personalisation in Weather-based Healthcare using Machine Learning
}

\author{
Radiah Haque ${ }^{1}$, Sin-Ban $\mathrm{Ho}^{1(\bullet)}{ }^{[0000-0003-2995-2120]}{ }^{\text {, Ian Chai }}{ }^{1}$, Chin-Wei Teoh ${ }^{1}$, Adina \\ Abdullah $^{2}$ [0000-0003-1545-7110], Chuie-Hong Tan ${ }^{3}$, and Khairi Shazwan Dollmat ${ }^{1}$ \\ ${ }^{1}$ Faculty of Computing and Informatics, Multimedia University, 63100 Cyberjaya, Malaysia \\ ${ }^{2}$ Department of Primary Care Medicine, Faculty of Medicine. University of Malaya, 50603 \\ Kuala Lumpur, Malaysia \\ ${ }^{3}$ Faculty of Management, Multimedia University, 63100 Cyberjaya, Malaysia \\ \{sbho, ianchai, shazwan.dollmat\}@mmu.edu.my, radumoni2@gmail.com, \\ cwtewen@gmail.com, adina@ummc.edu.my, chtan@mmu.edu.my
}

\begin{abstract}
Enhancing personalisation is important for productive collaboration between humans and machines. This is because the integration of human intelligence with cognitive computing would provide added value to healthcare. While the wellbeing and human health can be profoundly affected by weather, the effect of machine learning on personalised weather-based healthcare for self-management is unclear. This paper seeks to understand how machine learning use affects the personalisation of weather-based healthcare. Based on the Uses and Gratifications Theory (UGT), new constructs are incorporated (demography, weather and effectiveness) in order to propose a model for health science with machine learning use, weather-based healthcare, and personalisation. Subsequently, this paper proposes building a system that can predict the symptoms of two diseases (asthma and eczema) based on weather triggers. The outcome from this paper will provide deeper understanding of how personalisation is impacted by machine learning usage and weather-based healthcare for individual patients' self-management and early prevention. The findings in this paper will also assist machine learning facilitators design effective use policies for weather-based healthcare that will have new fundamental knowledge with personalisation to enhance the future of intelligent health informatics, and artificial intelligence.
\end{abstract}

Keywords: Machine Learning, Intelligent Health Informatics, Artificial Intelligence, Weatherbased Healthcare, Mobile Application. 
ABSTRACTS

\title{
A CNN-based Model for Early Melanoma Detection
}

\author{
Amer Sallam $^{1}$, Abdulfattah E. Ba Alawi², Ahmed Y. A. Saeed ${ }^{2}$ \\ ${ }^{1}$ Computer Network and Distributed Systems Department, Taiz University, Taiz, Yemen \\ ${ }^{2}$ Software Engineering Department, Taiz University, Taiz, Yemen \\ amer.sallametaiz.edu.ye, baalawi.abdulfattah@gmail.com, \\ ahmed12.12f@gmail.com
}

\begin{abstract}
Melanoma is a serious form of skin cancer that develops from pigment-producing cells known as melanocytes, which in turn produce melanin that gives your skin its color. Early detection of these symptoms will certainly help affected people to overcome their suffering and find appropriate solutions for their treatment methods. That is why researchers have tried in many studies to provide technical solutions to help early detection of skin cancer. In this paper, a smart pre-trained model based on deep learning techniques for the early detection of Melanoma and Nevus has been proposed. It is designed to track and divide the dynamic features of the dermoscopic ISIC dataset into two distinguished classes Melanoma and Nevus of epidermal pathologies. AlexNet and GoogLeNet are used to classify each cancer type according to their profile features. It was found that the average classification accuracy for the above-mentioned algorithms is $90.2 \%$ and $89 \%$ respectively, providing plausible results when comparing to other existing models.
\end{abstract}

Keywords: Skin Diseases, Dermoscopic, Dermatologist, CAD, Melanoma, GoogLeNet, AlexNet. 
ABSTRACTS

\title{
SMARTS D4D Application Module for Dietary Adherence Self-Monitoring among Hemodialysis Patients
}

\author{
Hafzan Yusoff ${ }^{1}$ Nur Intan Raihana Ruhaiyem ${ }^{2}$ and Mohd Hakim Zakaria ${ }^{1}$ \\ ${ }^{1}$ School of Health Sciences, Universiti Sains Malaysia, 16150 Kota Bharu, Kelantan, Malaysia \\ ${ }^{2}$ School of Computer Sciences, Universiti Sains Malaysia, 11800, USM, Penang, Malaysia \\ hafzanyeusm.my
}

\begin{abstract}
The mortality rate in hemodialysis patients is 6.3-8.2 times higher than the general population. Failure to adhere to dietary intake recommendation, was one of the most significant factors affecting patient survival. Technology-mediated approach such as web and mobile application could be the most desirable approach nowadays. This paper presents the SMARTS dual application modules development by using ADDIE model, begining with the analysis of needs, followed by content and face validation in the design phase, and finally the development of application prototype. The application system was designed to enable seamless access, interaction, and monitoring between all the involved users; patient, caretaker, and Healthcare Provider (HCP). Twenty-five respondents involved in the need assessment and also face and validity testing, Most of them are dietitian from government hospital $(n=16,64 \%)$, university medical centres $(n=6,24 \%)$ and private hospital $(n=2,8 \%)$, with ample experience managing hemodialysis patients. Majority of them rated the content (84\%), and purpose of the app as a new nutrition education tool (84\%) as the most appealing properties of the app, followed by the visual appealing (68\%), and variety of topics offered $(40 \%)$. Some improvisation was suggested on the comprehension and quality of the text, inclusion of nutrient tracker, presentation of education messages in video format, and adding more visuals rather than textual information to enhance understanding. The SMARTS D4D module was well-accepted and supportive of respondents' needs. Appropriate modifications have been done based on the valuable respondents' feedbacks.
\end{abstract}

Keywords: Hemodialysis, Diet, Application Module. 
ABSTRACTS

\title{
Improved Multi-Label Medical Text Classification using Features Cooperation
}

\author{
Rim Chaib ${ }^{1,2}$, Nabiha Azizi ${ }^{1,2}$, Nawel Zemmal ${ }^{1,3}$, Didier Schwab ${ }^{4}$ and Samir Brahim \\ Belhaouari $^{5}$ \\ ${ }^{1}$ Labged Laboratory of electronic document management \\ ${ }^{2}$ Computer science department, Badji Mokhtar University, Annaba, 23000. Algeria \\ ${ }^{3}$ Department of Mathematics and Computer Science, Mohamed Cherif Messaadia University, \\ Souk-Ahras, 41000. Algeria. \\ ${ }^{4}$ LIG-GETALP Laboratory, Grenoble Alpes University, Grenoble, France \\ ${ }^{5}$ College of Science and Engineering, Hamad Bin Khalifa University, Doha, Qatar \\ azizi@labged.net, chaib.rim23@gmail.com
}

\begin{abstract}
Medical text categorization is a valuable area of text classification due to the massive growth in the amount of medical data, most of which is unstructured. Reading and understanding the information contained in millions of medical documents is a time-consuming process. Automatic text classification aims to automatically classify text documents into one or more predefined categories according to several criteria such as the type of output (multi-label or mono label). Feature extraction task plays an important role in text classification. Extracting informative features highly increases the performance of the classification models and reduces the computational complexity. Traditional feature extraction methods are based on handcrafted features which mainly depend on prior knowledge. The use of these features may involve an insignificant representation. Doc2 vec is a way to generate a vector of informative and essential features that are specific to a document. In this paper, the impact of combining handcrafted and doc $2 \mathrm{vec}$ features in the multi-label document classification scenario is analyzed by proposing a system named MUL-MEDTEC. The one-versus-all classification strategy based on logistic regression is adopted in this study to predict for each medical text it to one or several labels. Experimental results based on Ohsumed medical dataset are very encouraging with based classification accuracy equal to 0.92 as global precision.
\end{abstract}

Keywords: text categorization, multi-label classification, medical text, handcrafted features, doc2vec. 
ABSTRACTS

\title{
Image Modeling through Augmented Reality for Skin Allergies Recognition
}

\author{
Nur Intan Raihana Ruhaiyem and Nur Amalina Mazlan \\ School of Computer Sciences, Universiti Sains Malaysia, 11800, USM, Penang, Malaysia \\ intanraihana@usm.my
}

\begin{abstract}
Skin rashes and allergies are common on human body. To date, we could find many skin care products sold not only in pharmacy but also from individual business. However, not all products suitable for all skin types. As a normal human, we sometimes not know the type of rashes or allergies that we faced. Meeting dermatologist would not be the first choice for many patients - given that the fees are expensive especially. Skin rashes can occur to anybody and an early recognition could avoid the rash become worse. Seeking information online would be the first choice, however patients still in high possibilities in mistakenly buy skin care products. Therefore, the development of the augmented reality application for skin rashes and allergies detection is expected can solve the problem. With the help of dermatologist and healthcare people, the information in this application is established and trustable. Among the advantages of this application are the ability in detecting of different types of skin rashes, displaying informative details on the detected skin rashes to reduce wrong judgement on the allergies the patient faced, and reasonable processing speed on mobile screen.
\end{abstract}

Keywords: Augmented reality, Skin rashes, Image processing, 3D modeling, Mobile application. 
ABSTRACTS

\title{
Hybridisation of Optimised Support Vector Machine and Artificial Neural Network for Diabetic Retinopathy Classification
}

\author{
Nur Izzati Ab Kader, Umi Kalsom Yusof and Maziani Sabudin \\ School of Computer Sciences, Universiti Sains Malaysia, 11800 Pulau Pinang, Malaysia \\ nurizzati.ucom13estudent.usm.my, umiyusof@usm.my, mazi- \\ anieusm.my
}

\begin{abstract}
Diabetic Retinopathy (DR) is a threatening disease which causes blindness in diabetic patients. With the increasing number of DR cases, diabetic eye screening is a challenging task for experts. Adopting machine learning to create a high accuracy classifier will be able to reduce the burden of diabetic eye screening. Therefore, this paper aims to propose a high accuracy DR classifier using clinical attributes. This study was conducted using nine clinical attributes of 385 diabetic patients, who were already labelled regarding DR, where 79 patients did not suffer from DR (NODR), 161 patients had nonproliferative DR (NPDR), and 145 patients had proliferative DR (PDR). The data was then used to develop a DR classifier through the hybrid of optimised Support Vector Machine (SVM) and Artificial Neural Network (ANN). The experiment results showed that the hybrid classifier had a high accuracy of 94.55. The accuracy yield was higher compared to single classifier.
\end{abstract}

Keywords: Diabetic Retinopathy, Classification, Hybridisation, Support Vector Machine, Neural Network 


\title{
A Habit-Change Support Web-Based System with Big Data Analytical Features for Hospitals (Doctive)
}

\author{
Cheryll Anne Augustine and Pantea Keikhosrokiani \\ School of Computer Sciences, Universiti Sains Malaysia, 11800 Minden, Penang, Malaysia \\ pantea@usm.my
}

\begin{abstract}
Even with the advancement of medical services, we still see an increase in mortality rate around the world especially due to heart disease, where it constantly remains as the number one cause of death globally. In order for an individual to protect their health, they are required to adopt healthy eating and practice regular exercises which also means that they have to adapt to a habit change in their daily routine. This healthy habit change does not only protect against heart diseases but also other chronic diseases such as cancer and stroke. Therefore, a habit-change support web-based system with big data analytics and decision-making features called Doctive is developed in this study to lower the risks of heart diseases. Doctive is targeted for hospital authorities to monitor patients and their habits and to prescribe medication and advice based on patients' habits and gathered information. Furthermore, this system also provides emergency assistance for patients based on their current location. This proposed system, would also be beneficial in collecting and organizing patients' information to ease access and speed the process of data entry and retrieval. The system was tested and evaluated by 5 people who were medically qualified or with knowledge and expertise in the field of data analytics and visualization. After gathering their opinionated responses, the results were tabulated and analyzed to be taken into consideration for improvements and to garner ideas for the future development of the system. Doctive can be useful for healthcare providers and developers.
\end{abstract}

Keywords: Habit-Change, Medical Information System, Web-based System, Big Data Analytics, Decision-Making. 
ABSTRACTS

\title{
An Architecture for Intelligent Diagnosing Diabetic Types and Complications Based on Symptoms
}

\author{
Gunasekar Thangarasu ${ }^{1}$, P.D.D. Dominic ${ }^{2}$ and Kayalvizhi Subramanian ${ }^{3}$ \\ ${ }^{1}$ Department of Professional Industry Driven Education, MAHSA University, Malaysia \\ ${ }^{2}$ Department of Computer and Information Science, University Technology PETRONAS, \\ Malaysia \\ ${ }^{3}$ Department of Fundamental and Applied Sciences, University Technology PETRONAS, \\ Malaysia \\ gunasekar97@gmail.com, dhanapal_deutp.edu.my and \\ skayalvizhi2012@gmail.com
}

\begin{abstract}
Information and communication technology can play a vital role in improving healthcare services by providing new and efficient ways of diagnosing diseases. Diabetic is recognized as the fastest-growing disease in the world. Due to insufficient diagnostic mechanisms, the number of undiagnosed diabetes has been increasing day by day. And it leads to creating long term complications such as neuropathy, nephropathy, foot gangrene and so on. The objective of this study is to design an intelligent architecture for diagnosing diabetes effectively based on the individual physical symptoms. The architecture has been designed by utilizing the combination of neural networks, data clustering algorithms and fuzzy logic techniques. Subsequently, a prototype system has been developed to validate against the diagnostic architecture on the aspect of efficiency and accuracy of diagnosing diabetes, and its types and complications. The overall qualitative findings from this study scored very high, which is 94.50 $\%$ accurate.
\end{abstract}

Keywords: Diabetes, Complications, Neural Networks, Fuzzy logics and Clustering. 


\title{
An Advanced Encryption Cryptographically-Based Securing Applicative Protocols MQTT and CoAP to Optimize Medical-IOT Supervising Platforms
}

\author{
Sanaa EL AIDI ${ }^{1}$, Abderrahim Bajit ${ }^{1}$, Anass BARODI ${ }^{1}$, Habiba CHAOUI ${ }^{1}$ and Ahmed \\ Tamtaoui $^{2}$ \\ ${ }^{1}$ Laboratory of Advanced Systems Engineering (ISA), National School of Applied Sciences, \\ Ibn Tofail University. Kenitra, Morocco. \\ ${ }^{2}$ National Institute of Posts and Telecommunications (INPT-Rabat), SC Department, Moham- \\ med V University. Rabat, Morocco.

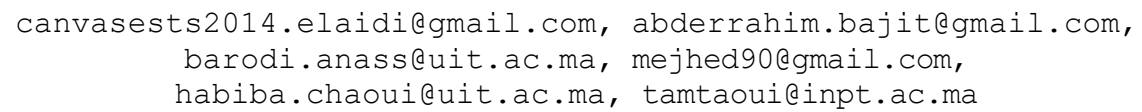

\begin{abstract}
Our proposed Platform is to detect and to measure the temperature of persons with PIR Node IOT, and then verify his identity through the combination of an RFID Node IOT and facial recognition with Cam Node IOT and if these tests are valid, the persons can then access the public area. With the security layer of the CoAP (Constrained Application Protocol) and MQTT(Message Queuing Telemetry Transport) communication protocols to compare these 2 protocols in terms of the execution time, the RAM memory space occupation, and the execution CPU consumptions. Then we have able to create an intelligent and secure medical IoT Platform this has been designed to monitor citizens to access this vast area in a more organized and secure manner to reduce the severity of this pandemic.
\end{abstract}

Keywords: MQTT, MQTT Client IOT , CoAP Client IOT , Broker, CoAP SERVER IOT , AES Encryption, CoAP, IoT, Artificial Intelligence, microcontroller, OpenCV 
ABSTRACTS

\title{
Pulmonary Nodule Classification Based On Three Convolutional Neural Networks Models
}

\author{
Enoumayri Elhoussaine and Belaqziz Salwa \\ LabSIV Laboratory, Department of Computer Science, Faculty of Science, Ibn Zohr \\ University, BP 8106, 80000 Agadir, Morocco \\ h.enoumayri@gmail.com, s.belaqziz@uiz.ac.ma
}

\begin{abstract}
Lung cancer is one among the leading reason for cancer-related death worldwide. To plan effective treatment, create monetary and care plans, early diagnosing of lung nodules in computed tomography (CT) chest scans must be performed. In this context, the purpose of this paper is to take into account the problem of classification between malignant and benign pulmonary nodules in CT scans, which aims to automatically map 3D nodules to category labels. Thus, we propose an ensemble learning approach based on three Convolutional Neural Networks including a basic 3D CNN, a 3D model inspired by AlexNet, and another 3D model inspired by ResNet. The result from these CNNs is combined to estimate one result, using a fully connected layer with a softmax activation. These CNNs are trained and evaluated on LIDC-IDRI public dataset. The best result is obtained by the ensemble model, providing a larger area under the receiver operating characteristic (ROC) curve; $84.66 \%$ for AUC and $94.44 \%$ for sensitivity, with a data augmentation technique.
\end{abstract}

Keywords: Pulmonary nodule classification, LIDC-IDRI, Deep Neural networks, AlexNet, ResNet 
ABSTRACTS

\title{
A Comparative Study on Liver Tumor Detection Using CT Images
}

\author{
Abdulfattah E. Ba Alawi, Ahmed Y. A. Saeed, Borhan M. N. Radman, and Burhan T. \\ Alzekri \\ Software Engineering Department, Taiz University, Taiz, Yemen \\ baalawi.abdulfattah@gmail.com, ahmed12.12f@gmail.com, \\ borhanpfaloubidy91@gmail.com, burhanalzekri77@gmail.com
}

\begin{abstract}
Liver cancer (LC) is a globally known issue as one of the most common cancers among humans. It is deadly cancer, especially in developing countries. There are many algorithms that have been used to perform the detection of liver cancer with the help of both traditional machine learning classifiers and deep learning classifiers. To analyze the performance of commonly used algorithms, this paper introduces a comparative study on LC detection. The study includes both machine learning and deep learning techniques and more accurate methods for liver and tumor detection from CT images. With the advances in artificial intelligence (AI) with a convolution neural networks algorithm, the included methods in this comparative study achieved great results. The best accuracy among traditional machine learning classifiers reaches $98.9 \%$ using K-Nearest Neighborhood (KNN) classifier. The Xception pre-trained model obtained $99.7 \%$ in term of testing accuracy as the best classifiers among deep learning models. The performance of deep learning models is very promising to take place in medical decisions.
\end{abstract}

Keywords: Liver Tumor, CT scan, CNN, Pre-trained Model, Deep Learning 
ABSTRACTS

\title{
Brain Tumor Diagnosis System Based on RM Images: A Comparative Study
}

\author{
Ahmed Y. A. Saeed, Abdulfattah E. Ba Alawi, Borhan M. N. Radman \\ Software Engineering Department, Taiz University, Taiz, Yemen \\ ahmed12.12f@gmail.com, baalawi.abdulfattah@gmail.com, \\ borhanpfaloubidy91@gmail.com
}

\begin{abstract}
Cancers or tumors have their impact effects on humans, especially if the cancer is localized in an important organ such as the brain. It is important to detect cancer earlier so that many lives can be saved. As cancer diagnosis is highly timeconsuming and needs expensive tools, there is an immediate requirement to develop non-invasive, cost-effective, and efficient tools for brain cancer staging and detection. Brain scans that are commonly used are magnetic resonance imaging (MRI) and computed tomography (CT). In this paper, we studied the common algorithms that are used for brain tumor detection using imaging modalities of brain cancer and automatic computer-assisted methods. The main objective of this paper is to make a comparative analysis of several methods of detecting tumors in the Central Nervous System (CNS). The results of the applied classifiers are compared and analyzed using different metrics including accuracy, precision, and recall. The best accuracy reached using machine learning algorithms is $85.56 \%$ accuracy with Random Forest, while the best classifier among applied deep learning algorithms is Inception V4 with $99.6 \%$.
\end{abstract}

Keywords: brain cancer, central nervous system tumor, pathophysiology, deep learning, ResNet, ResNext. 
ABSTRACTS

\title{
Diagnosis of COVID-19 Disease Using Convolutional Neural Network Models based Transfer Learning
}

\author{
Hicham Moujahid ${ }^{1}$, Bouchaib Cherradi ${ }^{1,2}$, Mohammed Al-Sarem ${ }^{3}$ and Lhoussain \\ Bahatti $^{1}$ \\ ${ }^{1}$ SSDIA Laboratory, ENSET of Mohammedia, Hassan II University of Casablanca, 28820, \\ Mohammedia, Morocco. \\ ${ }^{2}$ STIE Team, CRMEF Casablanca-Settat, provincial section of El Jadida, 24000, El Jadida, \\ Morocco. \\ ${ }^{3}$ Information Systems Department, Taibah University, Al-Madinah Al-Monawarah, Kingdom \\ of Saudi Arabia. \\ \{hicham88moujahid, bouchaib. cherradi, lbahatti, mohsarem\} @gmail.com
}

\begin{abstract}
COVID-19 disease is similar to normal pneumonia caused by bacteria or other viruses. Therefore, the manual classification of lung diseases is very hard to discover, particularly the distinction between COVID-19 and NON-COVID-19 disease. COVID-19 causes infections on one or both lungs which appear as inflammations across lung cells. This can lead to dangerous complications that might cause death in the case of gaining or having an immune disease. The problem of COVID-19 is that its symptoms are similar to conventional chest respiratory diseases like flu disease and chest pain while breathing or coughing produces mucus, high fever, absence of appetite, abdominal pain, vomiting, and diarrhea. In most cases, a deep manual analysis of the chest's X-ray or computed tomography (CT) image can lead to an authentic diagnosis of COVID-19. Otherwise, manual analysis is not sufficient to distinguish between pneumonia and COVID-19 disease. Thus, specialists need additional expensive tools to confirm their initial hypothesis or diagnosis using real-time polymerase chain reaction (RT-PCR) test or MRI imaging. However, a traditional diagnosis of COVID-19 or other pneumonia takes a lot of time from specialists, which is so significant parameter in the case of a pandemic, whereas, a lot of patients are surcharging hospital services. In such a case, an automatic method for analyzing X-ray chest images is needed. In this regard, the research work has taken advantage of proposing a convolutional neural network method for COVID-19 and pneumonia classification. The X-ray processing have been chosen as a diagnosis way because of its availability in hospitals as a cheap imaging tool compared to other technologies. In this work, three CNN models based on VGG-16, VGG19, and MobileNet were trained using the zero-shot transfer learning technique. The best results are obtained on VGG-19 based model: $96.97 \%$ accuracy, $100 \%$ precision, $100 \% \mathrm{~F} 1$ score, and $99 \%$ recall.
\end{abstract}

Keywords: Convolutional Neural Network, Transfer learning, COVID-19, Pneumonia, X-ray images. 
ABSTRACTS

\title{
Early Diagnosos of Parkinson's Using Dimensionality Reduction Techniques
}

\author{
Tariq Saeed Mian [0000-0003-2666-9223] \\ Department of IS, College of Computer Science and Engineering \\ Taibah University, Madinah Almunwarah, Saudi Arabia \\ tmian@taibahu.edu.sa
}

\begin{abstract}
Correct and early diagnosing Parkinson's Disease (PD) is vital as it enables the patient to receive the proper treatment as required for the current stage of the disease. Early diagnosis is crucial, as certain treatments, such as levodopa and carbidopa, have been proven to be more effective if given in the early stages of PD. At present the diagnosis of PD is solely based on the clinical assessment of a patient's motor symptoms. By this stage however, PD has developed to such an extent that irreversible neurological damage has already occurred, meaning the patient has no chance of recovering. By implementing the use of machine learning into the process of assessing a potential PD patient the disease can be detected and diagnosed at a much earlier stage, allowing for swift intervention, which increases the chance of PD not developing to such damaging levels in the patient. Machine Learning is a subfield of artificial intelligence that provides different technique to scientists, clinicians and patients to address and detect diseases like PD at early stage. The main symptom of PD is the vocal impairment that distinguishes from the normal person. In this study, we used a PD vocal based dataset that has 755 features The Principal Component Analysis (PCA) and Linear Discriminate Analysis (LDA) techniques are used to reduce the dimensionality of the available Parkinson's dataset to 8 optimal features. The study used four supervised machine learning algorithms, two algorithms are from the ensemble techniques, Random Forest, Adaboost Support Vector Machine and Logistic Regression. The Random Forest model with LDA and PCA shows the highest accuracy of $0.948 \%$ and $0.840 \%$ respectively.
\end{abstract}

Keywords: Parkinson's Disease, Early Detection, Machine Learning, Linear Discriminate Analysis, Dimensionality Reduction, Principal Component Analysis, Ensemble Methods, Random Forest, Adaboost Support Vector Machine, Logistic Regression 
ABSTRACTS

\title{
Detection of Cardiovascular Disease using Ensemble Machine Learning Techniques
}

\author{
Fizza Kashif and Umi Kalsom Yusof
}

School of Computer Sciences, Universiti Sains Malaysia, Penang 11800, Malaysia

fizza@student.usm.my, umiyusof@usm.my

\begin{abstract}
Cardiovascular diseases are a group of heart and blood vessel disorders that are common worldwide, taking millions of lives each year. Many factors contribute towards a person getting a positive diagnosis such as hypertension, diabetes, and cholesterol level, among others. It proves to be a challenge to accurately predict the presence of a cardiovascular disease as this is a complex task. Timely and correct diagnosis is considered a highly important area in the field of clinical research and healthcare. Vast troves of data are collected by the healthcare industry every year and using this data effectively can prove to be an invaluable asset to the field. Machine learning and ensemble classification models can be employed to accurately predict heart disease in patients. Data mining techniques presented in this paper make use of patient data, from the UCI machine learning repository, to build a classification model that can be applied to new cases for the detection of heart disease. An ensemble model made from random forest and support vector machine algorithms is proposed which reports an accuracy of $89 \%$ in detecting heart disease. This model is further compared with other algorithms based on evaluation metrics like precision, recall and F-1 score.
\end{abstract}

Keywords: Ensemble Classification Algorithm, Machine Learning, Cardiovascular Disease. 
ABSTRACTS

Health Information Management 
ABSTRACTS

\title{
Hospital Information System for Motivating Patient Loyalty: A Systematic Literature Review
}

\author{
Saleh Nasser Rashid Alismaili ${ }^{1}$ and Mohana Shanmugam ${ }^{2}$ \\ ${ }^{1}$ College of Informatics and Computing, Universiti Tenaga Nasional, Malaysia; Directorate of \\ Information Technology, Ministry of Health, Muscat, Sultanate of Oman. \\ ${ }^{2}$ College of Informatics and Computing, Universiti Tenaga Nasional, Malaysia \\ pt20712@utn. edu.my
}

\begin{abstract}
Healthcare service institutions (HIS) seeking to motivate patient loyalty have identified Hospital Information Systems (HIS) as a potential solution to gather, measure, and analyze the healthcare data necessary for this goal. The purpose of this systematic review of the literature is to reveal how prevalent the use of HIS with respect to motivating patient loyalty, and to investigate the efficacy of HIS in doing so. To generate data, published empirical studies and conference papers from the past five years were compiled from the following online databases: Scopus, ACM Digital Library, IEEE Xplore, ScienceDirect, and Emerald Insight. The search results indicate that, while the use of HIS in motivating patient loyalty is rare relative to other topics within the general field of HIS, HIS use have a significant positive impact on patient satisfaction, which is understood in the literature to be directly related to patient loyalty. There remains a gap in empirical studies on the direct application of HIS with the purpose of increasing patient loyalty. Future research may be required on the development of an HIS focused on motivating patient loyalty, which can be empirically tested in a real-world HSI setting.
\end{abstract}

Keywords: Hospital Information Systems, Patient Loyalty, Patient Satisfaction 
ABSTRACTS

\title{
Context Ontology for Smart Healthcare Systems
}

\author{
Salisu Garba Radziah Mohamad and Nor Azizah Saadon \\ School of Computing, Faculty of Engineering, Universiti Teknologi Malaysia, 81310 UTM, \\ Skudai, Johor, Malaysia \\ Salisu.garba@graduate.utm.my, \{radziahm, azizahsaadon\}@utm.my
}

\begin{abstract}
This paper proposes an improved Context Ontology for Smart Healthcare Systems. The main contribution of this work is the simplification, sufficiently expressiveness, and extendability of the smart healthcare context representation, in which only three contextual classes are required - compared to several classes in the related context ontologies. This is achieved by adapting the feature-oriented domain analysis (FODA) techniques of software product line (SPL) for domain analysis, and subsequently, the lightweight unified process for ontology building (UPON Lite) is used for ontology development. To validate the applicability of the proposed context ontology, sustAGE smart healthcare case study is used. It is found that the proposed context ontology can be used to sense, reason, and infer context information in various users, environments, and smart healthcare services. The ontology is useful for healthcare service designers and developers who require simple and consolidated ontology for complex context representation. This paper will benefit the smart healthcare service developers, service requesters as well as other researchers in the ontology-based context modeling domain.
\end{abstract}

Keywords: Ontology; smart healthcare; context ontology; healthcare service 


\title{
A Modified UTAUT Model for Hospital Information Systems Geared Towards Motivating Patient Loyalty
}

Saleh Nasser Rashid Alismaili ${ }^{1}$ and Mohana Shanmugam ${ }^{2}$

${ }^{1}$ College of Informatics and Computing, Universiti Tenaga Nasional, Malaysia; Directorate of Information Technology, Ministry of Health, Muscat, Sultanate of Oman.

${ }^{2}$ College of Informatics and Computing, Universiti Tenaga Nasional, Malaysia

pt20712@utn.edu.my

\begin{abstract}
Healthcare service institutions (HSIs) have sought ways to motivate patient loyalty in response to surging rates of medical tourism. Previous research indicates that Hospital Information System (HIS) is essential for HSIs to gather, measure, and analyze the massive amounts of data required to generate patient loyalty. There is currently no consensus on the factors that comprise HIS specifically geared towards motivating patient loyalty (HISPL). Furthermore, HIS requires full adoption by HSI staff to be effective. Thus, to reduce wastage of HSI resources, it is necessary to predict whether a given HIS specifically geared towards motivating patient loyalty is likely to be adopted. The purpose of this study is to reveal the factors that comprise HISPL and to modify the Unified Theory of Acceptance and Use of Technology (UTAUT) model to help predict the likelihood of an HISPL to be fully adopted by HSI staff. The results revealed that pertinent HISPL factors are capability, configurability, ease of use/help desk availability and competence (EU), and accessibility/shareability (AS). Using these factors, the UTAUT model was modified to fit the specific needs of HISPL. The modifications are theoretical and will have to be validated in future empirical studies.
\end{abstract}

Keywords: Hospital Information System, patient loyalty, UTAUT 
ABSTRACTS

\title{
Teamwork Communication in Healthcare: An Instrument (questionnaire) Validation Process
}

\author{
Wasef Matar ${ }^{1}$, Monther Aldwair ${ }^{2}$ \\ ${ }^{1}$ University of Petra, Amman, Jordan, \\ ${ }^{2}$ College of Technological Innovation, Zayed University, Abu Dhabi, UAE, \\ Wasef.matar@uop.edu.jo, aldwairi@zu.ac.ae
}

\begin{abstract}
Healthcare face many problems, one of these problems is embodied in teamwork communication systems, the current HISs lack of teamwork communication tools. To introduce a teamwork communication instrument (questionnaire) in healthcare which plays a key role in health information system area. The proposed a research model for this study applied a quantitative approach using a survey method. To formulate the problem a preliminary data was collected by survey method to test and introduce a validated instrument (questionnaire). This study proposed and validated an instrument (questionnaire) to be used in healthcare teamwork communication studies. The findings of this study will be contributed to teamwork communication in healthcare and will be a reference for any healthcare communication related study. This study is the first of its kind in Jordan and has added a new dimension in the teamwork communication in healthcare.
\end{abstract}

Keywords: Teamwork communication, Clinical Pathways, Instrument (questionnaire), communication tools, Clinical Pathways 
ABSTRACTS

\title{
Potential Benefits of Social Media to Healthcare: A Systematic Literature Review
}

\author{
Ghada Ahmad Abdelguiom ${ }^{1}$ and Noorminshah A.Iahad ${ }^{2}$ \\ ${ }^{1}$ School of Computing, Faculty of Engineering, Universiti Teknologi Malaysia \\ ${ }^{2}$ Azman Hashim International Business School (Information Systems), Universiti Teknologi \\ Malaysia \\ ghada.sudan@gmail.com, minshaheutm.my
}

\begin{abstract}
Social media offers a rich online experience, dynamic content, usability, and knowledge that attracts more users. The use of social media in the health sector is indeed attracting more and more attention. Over the last ten years, researchers have attempted various topics related to the health sector via social media that have contributed beneficially to the healthcare domain. There is a demand for a study to identify the potential benefits of social media to healthcare. Thus, this paper surveys research papers related to the social media platform in the healthcare domain that were published between the years (2014-2020). The primary objective of this study is to review the range, nature, and extent of current research activity on the role of social media in healthcare. Therefore, this paper outlines the recent approaches to the utilization of social media to provide solutions for health-related issues. Also, it discusses the role of social media in promoting health care services. The study addresses the key issues addressed in the latest research, provides an overview of their shortcomings, restrictions, and finally, outlines the opportunities for future research.
\end{abstract}

Keywords: Health care, social networks, digital communication, social media, eHealth, Web 2.0. 
ABSTRACTS

\title{
Exploring the Influence of Human-centered Design on User Experience in Health Informatics Sector: A Systematic Review
}

\author{
Lina Fatini Azmi ${ }^{1}$ and Norasnita Ahmad $^{2}$ \\ ${ }^{1}$ UTM Research Computing, Department of Deputy Vice-Chancellor (Research \& Innovation), \\ Universiti Teknologi Malaysia, 81310 Skudai, Johor, Malaysia \\ ${ }^{2}$ Azman Hashim International Business School, Universiti Teknologi Malaysia, 81310 Skudai, \\ Johor, Malaysia \\ lina.fatini@utm.my, norasnita@utm.my
}

\begin{abstract}
Integrating human-centered design (HCD) approach in healthcare informatics solution are changing the landscape of the e-services and e-satisfaction among the users. Major evolution of informatics system in healthcare organization helps to revolve the role of design by changing it into key element that centralize on user's capability on improving their e-service. Ample studies on implementing element of humanities into user experience-based design are now being adapted in order to enhance satisfaction and utmost benefits to users. This paper is built on a systematic literature review of academic papers that seeks to explore the influence of human-centered design approach towards user experience in health informatics sector. The total number of selected literatures using PRISMA process for this study is $n=64$. The obtained results of this study highlighted the relation between human-centered design approach and user experience. This study also illustrates the process of human-centered design flow adapted from selected studies focus on healthcare sector in a unique approach to developing user-friendly informatics system to bridge the user experience gap.
\end{abstract}

Keywords: Systematic review, Human-centered design, Health informatics system, Health Informatics sector. 
ABSTRACTS

\title{
An Emotional-Persuasive Habit-Change Support Mobile Application for Heart Disease Patients (BeHabit)
}

\author{
Bhavani Devi Ravichandran and Pantea Keikhosrokiani \\ School of Computer Sciences, Universiti Sains Malaysia, 11800 Minden, Penang, Malaysia \\ pantea@usm.my
}

\begin{abstract}
Heart disease is stated as the world's biggest killers. The risk factors of this deadly disease are due to some bad habits such as being overweight, bad eating diet, smoking, assumption of alcohol, etc. Nevertheless, patients can live a healthy lifestyle if they have the proper guidance of persuasive-emotional featured technologies. In line with this, this study focuses on developing an emotional-persuasive habit-change support mobile application called BeHabit to improve heart disease patients' lifestyles. Persuasive-emotional features are two different features that are integrated with BeHabit to distinguish this application from the existing ones. The proposed system is designed, implemented, tested, and evaluated by 10 users. In conclusion, the users are satisfied to used BeHabit to change their bad habits. Emotional and persuasive features which are integrated into BeHabit are the key to help patients to change their bad habits. BeHabit and the integrated feature can be used as a guideline for healthcare developers and providers for the improvement of mHealth services.
\end{abstract}

Keywords: Heart disease, mHealth, Habit-change, Persuasive, Emotional features, Mood, Medical Information System. 
ABSTRACTS

\title{
A Systematic Review of the Integration of Motivational and Behavioural Theories in Game-based Health Interventions
}

\author{
Abdulsalam S. Mustafa, Nor'ashikin Ali, Jaspaljeet Singh Dhillon \\ Universiti Tenaga Nasional, Selangor, Malaysia \\ salamsmeyahoo.com, nora.alio8@uniten.edu.my, \\ jaspaljeet@uniten.edu.my
}

\begin{abstract}
M-Health interventions designed for healthcare can potentially increase participation and behaviour outcomes. However, interventions need to incorporate a theoretical perspective of behavioral change to enhance their perceived efficacy. Although behavioral outcome theories have gained interest in the health and fitness literature, the implementation of theoretical integration remains largely under-studied. Therefore, we reviewed the efficacy of behavioural gamified interventions based on integrated theories in various contexts, such as healthcare and fitness. Studies were included if integrated theoretical intervention was implemented to change behaviour in specific contexts. The review aims to uncover the effectiveness of integrated theory in predicting behaviour outcome in interventions. Our findings reveal that in 39 studies, Self Determination Theory $(n=19)$ and Theory of Planned Behaviour $(n=16)$ outnumbered other theories in integrated models. Overall, $77 \%$ of studies showed evidence that integrated theoretical-based behaviour change interventions can be successful for a short time, with only a few studies that tested the long-term effectiveness of these interventions. We discuss the implication of our findings, and also propose potential future directions.
\end{abstract}

Keywords: Integrated Theories, Hybrid, Gamification, Intervention, Behaviour Change, Health and Fitness. 
ABSTRACTS

\title{
Adopting React Personal Health Record (PHR) System in Yemen HealthCare Institutions
}

\author{
Ziad Saif Alrobieh ${ }^{1}$, Dhiaa Faisal Alshamy ${ }^{2}$, Maged Nasser ${ }^{3}$ \\ ${ }^{1}$ Department of Communication \& Computer Engineering, Alsaeed Faculty for Engineering \& \\ Information Technology, Taiz University, Taiz, Yemen \\ ${ }^{2}$ Department of Networking and Distributed Systems, Faculty of Information Technology and \\ Engineering, Taiz university, Taiz, Yemen \\ ${ }^{3}$ School of Computing, Universiti Teknologi Malaysia, Johor Bahru, Johor 81310, Malaysia. \\ ziadrh@yahoo.com, dhodhofegmail.com, \\ maged.m.nasser@gmail.com
}

\begin{abstract}
Health care is a critical sector of society that requires quality improvement of healthcare services, information technology IT systems have a great impact on improving the quality of these services, unfortunately, The effect of information culture on the implementation of information systems by healthcare providers in the developed countries is little known, considering the importance of information culture. Despite that many Yemeni healthcare facilities have been already using information systems to digitize the management of healthcare providing procedures, The patients' health information including disease history and prescriptions is not fully recorded and additionally, there is no implementation of Personal Health Record PHR systems where patients can access, and control their health records from another place where their records are stored locally in the healthcare providers' databases. The existing electronic health record systems are limited and do not exploit the available technology solutions and services. To explore the advantages of using PHR systems, multiple kinds of research are being studied and the proof of use has been cleared by these researchers' conclusions, also a survey was made; to ensure people intention to using PHR systems, and to observe their opinion on what they need to be provided by the system and what interests them. Designing the web-based system was done after going through the available platforms to choose the most correct and suitable solutions to assure that the system meets the requirements needed. Adopting innovative and modern technological solutions such as PHR web applications is a fine way to improve patient safety and quality of care, increase efficiency, Decision supporting, and Increase patient and health workers' satisfaction. The proposed solution ensured the patients' satisfaction and safety by giving them access to their health records whenever and wherever they are through their device's browser and helped the doctors to make the right decisions and speed up the healthcare providing process which reduced the damage caused by the current systems, saving human lives and preventing serious health issues.
\end{abstract}

Keywords: Health records systems, PHR systems, Healthcare, Technological solutions 
Artificial Intelligence and Soft Computing 


\title{
Application of Shuffled Frog-Leaping Algorithm for Optimal Software Project Scheduling and Staffing
}

\author{
Ahmed O. Ameen ${ }^{1}$, Hammed A. Mojeed ${ }^{1}$, Abdulazeez T. Bolariwa ${ }^{1}$, \\ Abdullateef O. Balogun ${ }^{1,2}$, Modinat A. Mabayoje ${ }^{1}$, Fatima E. Usman-Hamzah ${ }^{1}$ and \\ Muyideen Abdulraheem ${ }^{1}$ \\ ${ }^{1}$ Department of Computer Science, University of Ilorin, PMB 1515 Ilorin, Nigeria \\ ${ }^{2}$ Department of Computer and Information Sciences, Universiti Teknologi PETRONAS, \\ Bandar Seri Iskandar, 32610 Perak, Malaysia \\ aminamed@unilorin.edu.ng, mojeed.ha@unilorin.edu.ng, \\ bolarinwa346@gmail.com, balogun.aoleunilorin.edu.ng, \\ mabayoje.ma@unilorin.edu.ng, usman-hamza.fa@unilorin.edu.ng, \\ muyideen@unilorin.edu.ng, abdullateef_16005851@utp.edu.my
}

\begin{abstract}
Software Project Scheduling Problem is one of the most crucial issues in software development because it includes resources planning; cost estimates, staffing and cost control which if not properly planned affect the timely completion of the software project. Software project scheduling is a problem of scheduling the tasks (work packages) and employees in such a way that the overall project completion time is minimized without violating dependency constraints (tasks dependencies) and being consistent with resource constraints. This study adopts a Search Based Software Engineering approach that focuses on multi-objective optimization for a software project planning using the Shuffled Frog Leaping Algorithm, a memetic meta-heuristic algorithm. The objectives are optimal ordering of work packages without dependency violation and allocation of staff to the work packages such that only employee(s) with required competence(s) are allotted to a given work package. The study was carried out in four stages, namely: frog (solution) representation, definition of the fitness function, implementation of Shuffled Frog Leaping Algorithm and evaluation with a randomly generated Software Project Scheduling Problem. The study concludes that it is possible to find an efficient solution to a Software Project Scheduling Problem by implementing the SFLA than any other traditional computing means which are tedious, error prone and costly.
\end{abstract}

Keywords: Shuffled Frog-Leaping Algorithm, Software Project Scheduling Problem, Software project planning, Search Based Software Engineering. 


\title{
A Long Short Term Memory and a Discrete Wavelet Transform to Predict the Stock Price
}

\author{
Mu'tasem Jarrah ${ }^{1}$ and Naomie Salim ${ }^{2}$ \\ ${ }^{1}$ King Abdulaziz University - Jeddah, KSA \\ ${ }^{2}$ Universiti Teknologi Malaysia - UTM, Johor Bahru, Malaysia \\ mmjarrah@kau.edu.sa, naomie@utm.my
}

\begin{abstract}
Financial Analysis is a challenging task in the present-day world, where investment value and quality are paramount. This research work introduces the use of a prediction technique that uses a combination of Discrete Wavelet Transform (DWT) and Long Short-Term Memory (LSTM) to predict stock prices in the Saudi stock market for the subsequent seven days. A time series model is used where comprises the historical closing values of several stocks listed on the Saudi stock exchange. This model is called the Discrete Long Short-Term Memory (DLSTM) which comprises memory elements that preserve data for extended periods. The function determined the historical closing price of the stock market and then employed Autoregressive Integrated Moving Average (ARIMA) for analysis. The DLSTM-based experimental model had a prediction accuracy of $97.54 \%$, while that of ARIMA was $97.29 \%$. The results indicate that DLSTM is an effective tool for predicting the prices in the stock market. The results highlight the importance of deep learning and the concurrent use of several information sources to predict stock price levels
\end{abstract}

Keywords: Long Short Term Memory, deep learning, prediction, stock market. 
ABSTRACTS

\title{
Effective Web Service Classification Using a Hybrid of Ontology Generation and Machine Learning Algorithm
}

\author{
Murtoza Monzur, Radziah Mohamad and Nor Azizah Saadon \\ School of Computing, Faculty of Engineering, Universiti Teknologi Malaysia UTM, 81310 \\ Skudai, Johor, Malaysia \\ muamurtoza2@graduate.utm.my
}

\begin{abstract}
Efficient and fast service discovery becomes an extremely challenging task due to the proliferation and availability of functionally-similar web services. Service classification or service grouping is a popular and widely applied technique to classify services into several groups according to similarity, in order to ease up and expedite the discovery process. Existing research on web service classification uses several techniques, approaches and frameworks for web service classification. This study focused on a hybrid service classification approach based on a combination of ontology generation and machine learning algorithm, in order to gain more speed and accuracy during the classification process. Ontology generation is applied to capture the similarity between complicated words. Then, two machine learning classification algorithms, namely, Support Vector Machines (SVMs) and Naive Bayes (NB), were applied for classifying services according to their functionality. The experimental results showed significant improvement in terms of accuracy, precision and recall. The hybrid approach of ontology generation and NB algorithm achieved an accuracy of 94.50\%, a precision of $93.00 \%$ and a recall of $95.00 \%$. Therefore, a hybrid approach of ontology generation and NB has the potential to pave the way for efficient and accurate service classification and discovery.
\end{abstract}

Keywords: Web Service Discovery, Web Service Description Language (WSDL), Service Classification, Ontology, Machine Learning, Support Vector Machines (SVMs), Naive Bayes (NB). 


\title{
Binary Cuckoo Optimisation Algorithm and Information Theory for Filter-Based Feature Selection
}

\author{
Ali Muhammad Usman ${ }^{1,2}$, Umi Kalsom Yusof ${ }^{1}$ and Syibrah Naim ${ }^{3}$ \\ ${ }^{1}$ School of Computer Sciences, Universiti Sains Malaysia, 11800 Pulau Pinang, Malaysia. \\ ${ }^{2}$ Department of Computer Sciences, Federal College of Education (Technical), Gombe, \\ Nigeria. \\ ${ }^{3}$ Technology Department, Endicott College of International Studies (ECIS), Woosong \\ University, Korea. \\ aliakko@yahoo.com, umiyusof@usm.my, syibrah@wsu.ac.kr
}

\begin{abstract}
Dimensionality reduction is among the data mining process that is used to reduce the noise and complexity of the features. Feature selection (FS) is a typical dimensionality reduction that is used to reduce the unwanted features from the datasets. FS can be either filter or wrapper. Filters lack interaction among selected subsets of features which in turns affect the classification performance of the chosen subsets of features. This study proposes two ideas of information theory entropy (E) as well as mutual information (MI). Both of them were used together with binary cuckoo optimisation algorithm BCOA (BCOA-E and BCOA-MI) to reduce both the error rate and computational complexity on four different datasets. A support vector machine classifier was used to measure the error rates. The results are in favour of BCOA-E in terms of accuracy. In contrast, BCOA-MI is computationally faster than BCOA-E. Comparison with other approaches found in the literature shows that the proposed methods performed better in terms of accuracy, number of selected features and execution time.
\end{abstract}

Keywords: Feature Selection, Filter-Based, Binary Cuckoo Optimisation, Information Theory. 
ABSTRACTS

\title{
Optimized Text Classification using Correlated Based Improved Genetic Algorithm
}

\author{
Thabit Sabbah
}

Al Quds Open University (QOU), Ramallah, Palestine

tazazmeh@qou.edu

\begin{abstract}
Text Classification (TC) is one of the basic processes in many Information Retrieval systems. Still, the performance of TC is a subject of improvement, and many approaches were proposes to achieve this aim. This work proposes an Improved Genetic algorithm (IGA) inspired by Genetic Engineering to enhance TC performance. In IGA, chromosome generation process were re-designed to diminish the effect of correlated genes. The Support Vector Machine (SVM) classifier were utilized based on the "Sport Text" popular dataset to evaluate the proposed approach. Empirical classification results were improved using IGA as compared to normal GA optimization. The proposed Improved Genetic Algorithm (IGA) improved the correct rates of TC by $1.39 \%$ in average.
\end{abstract}

Keywords: Text Classification, Improved Genetic Algorithm, Genetic Engineering, Feature correlation. 


\title{
Multi-objective NPO Minimizing the Total Cost and $\mathrm{CO}_{2}$ Emissions for a Stand-Alone Hybrid Energy System
}

\author{
Abbas Q. Mohammed ${ }^{1,2}$ 、 Kassim A. Al-Anbarri ${ }^{1}$, Rafid M. Hannun ${ }^{3}$ \\ ${ }^{1}$ Electrical Engineering Department, Faculty of Engineering, Mustansiriyah University, \\ Baghdad, Iraq. \\ ${ }^{2}$ Construction and Projects Department, University of Thi-Qar, Nassriyah, Thi-Qar, Iraq. \\ ${ }^{3}$ Mechanical Engineering, College of Engineering, University of Thi-Qar, Nassriyah, Thi- \\ Qar, Iraq. \\ abbasqassimeng@gmail.com
}

\begin{abstract}
This article proposes a new algorithm called Nomadic People Optimizer (NPO) to find the optimal sizing of a hybrid energy system (HES), consisting of photovoltaic cell (PV), battery storage (BS), and diesel generator (DG). The HES supply the electricity to an academic building located in Thi-Qar Province, which is located in southern Iraq on latitude $31.06^{\circ}$ and longitude $46.26^{\circ}$. The objectives of this algorithm are to reduce the total cost during the life cycle of the project, and this is an economic aspect that in turn reduces energy costs, the second goal is to reduce emissions of carbon dioxide. While continuing to supply the electrical load with electricity throughout the life cycle of the project for 25 years. The results show that optimal sizing of the HES achieved by 1875 number of the PV,687 number of the BS, and single DG
\end{abstract}

Keywords: Renewable Energy, Solar Energy, Nomadic People Optimizer, Optimization. 


\title{
A Real time Flood Detection System Based on Machine Learning Algorithms
}

\author{
Abdirahman Osman Hashi ${ }^{1,3}$, Abdullahi Ahmed Abdirahman ${ }^{1}$, Mohamed \\ Abdirahman Elmi ${ }^{1}$, Siti Zaiton Mohd Hashim ${ }^{2}$ \\ ${ }^{1}$ Faculty of Computing, SIMAD University, Mogadishu-Somalia. \\ ${ }^{2}$ Department of Artificail intelligence and Big Data, Faculty of Computing, Universiti Malaysia \\ Kelantan, 16100 Pengkalan Chepa, Kelantan, Malaysia \\ ${ }^{3}$ Department of computer Science, Faculty of Informatics, istanbul Teknik Üniversitesi , 34469 \\ Masklak, İstanbul, Turkey \\ Wadani12727@gmail.com; aaayare@simad.edu.so; sitizaiton@utm.my; \\ m.abdirahmanesimad.edu.so
}

\begin{abstract}
Flood is expressed as water overflowing onto the ground that usually is dry or an increase of water that has a significant impact on human life and it is also declared as one of the most usually natural phenomenon, causing severe financial crisis to goods and properties as well as affecting human lives. However, preventing such floods would be useful to the inhabitants in order to get a sufficient time to evacuate in the areas that might be possible floods can happen before the actual floods happen. To address the issue of floods, many scholars' proposed different solutions such as developing prediction models and building a proper infrastructure. Nevertheless, these proposed solutions are not efficient from an economic perspective in here, Somalia. Therefore, the key objective of this research paper is to intend a new robust model which is a real-time flood detection system based on Machine-Learning-algorithms; RandomForest, Naïve-Bayes and J48 that can detect water level and measure floods with possible humanitarian consequences before they occur. The experimental results of this proposed method will be the solution of forth mentioned problems and conduct research on how it can be easily simulate a novel way that detects water levels using hybrid model based on Arduino with GSM modems. Based on the analysis, RandomForest-algorithm were outperformed other machine-learning-methods in-terms of accuracy over other-classification with $98.7 \%$ accuracy in-comparison with $88.4 \%$ and $84.2 \%$ for NaiveBayes and J48 respectively. The proposed method has contribution to the field of study by introducing a new way of preventing floods in the field of Artificial, data mining.
\end{abstract}

Keywords : Machine Learning, Naive Bayes, Random Forest, Artificial Intelligence, Data Mining. 
ABSTRACTS

\title{
Extracting Semantic Concepts and Relations from Scientific Publications by Using Deep Learning
}

\author{
Fatima N. AL-Aswadi ${ }^{1,2[0000-0001-5413-1207]}$, Huah Yong Chan ${ }^{10000-0002-2186-6597]}$ and Keng \\ Hoon Gan ${ }^{1}$ [0000-0002-2972-3523] \\ ${ }^{1}$ School of Computer Sciences, Universiti Sains Malaysia, 11800 Gelugor, Pulau Pinang, \\ Malaysia \\ ${ }^{2}$ Faculty of Computer Sciences and Engineering, Hodeidah University, Hodeidah, Yemen \\ fatima7aswadi@gmail.com, hychan@usm.my, khgan@usm.my
}

\begin{abstract}
With the large volume of unstructured data that increases constantly on the web, the motivation of representing the knowledge in this data in the machine-understandable form is increased. Ontology is one of the major cornerstones of representing the information in a more meaningful way on the semantic Web. The current ontology repositories are quite limited either for their scope or for currentness. In addition, the current ontology extraction systems have many shortcomings and drawbacks, such as using a small dataset, depending on a large amount predefined patterns to extract semantic relations, and extracting a very few types of relations. The aim of this paper is to introduce a proposal of automatically extracting semantic concepts and relations from scientific publications. This paper introduces a novel relevance measurement for concepts, and it suggests new types of semantic relations. Also, it points out of using deep learning (DL) models for semantic relation extraction.
\end{abstract}

Keywords: Concept Extraction, Deep Learning, Ontology Construction, Relevance Measurements, Semantic Relation Discovery. 
ABSTRACTS

\title{
Effectiveness of Convolutional Neural Network Models in Classifying Agricultural Threats
}

\author{
Sayem Rahman, Murtoza Monzur, and Nor Bahiah Ahmad \\ School of Computing, Faculty of Engineering, Universiti Teknologi Malaysia UTM, 81310 \\ Skudai, Johor, Malaysia \\ muamurtoza2@graduate.utm.my, rsayem2@graduate.utm.my
}

\begin{abstract}
Smart farming has recently been gaining traction for more productive and effective farming. However, pests like monkeys and birds are always a potential threat for agricultural goods, primarily due to their nature of destroying and feeding on the crops. Traditional ways of deterring these threats are no longer useful. The use of highly effective deep learning models can pave a new way for the growth of smart farming. This study aims to investigate the manner in which deep learning convolutional neural network (CNN) models can be applied to classify birds and monkeys in agricultural environments. The performance of CNN models in this case is also investigated. In this regard, four CNN variants, namely, VGG16, VGG19, InceptionV3 and ResNet50, have been used. Experiments were conducted on two datasets. The experimental results demonstrate that all the models have the capability to perform classification in different situations. Data quality, parameters of the models, used hardware during experiments also influence the performance of the considered models. It was also found that the convolutional layers of the models play a vital role on classification performance. The experimental results achieved will assist smart farming in opening new possibilities that may help a country's agriculture industry, where efficient classification and detection of threats are of potential importance.
\end{abstract}

Keywords: Smart Farming, Convolutional Neural Network (CNN), Deep Learning, Computer Vision, Image Processing. 
ABSTRACTS

\title{
A Study on Emotion Identification from Music Lyrics
}

\author{
Affreen Ara ${ }^{1}$ and Raju G. ${ }^{2}[0000-0002-7871-1801]$ \\ ${ }^{1}$ Department of Computer Science and Engineering, Christ (Deemed to be University), \\ Bengaluru, India. \\ ${ }^{2}$ Department of Data Science, Christ (Deemed to be University), Lavasa Campus, \\ Pune, India \\ affreen. araeres. christuniversity.in, \\ raju.g@christuniversity.in
}

\begin{abstract}
The widespread availability of digital music on the internet has led to the development of intelligent tools for browsing and searching for music databases. Music emotion recognition (MER) is gaining significant attention nowadays in the scientific community. Emotion Analysis in music lyrics is analyzing a piece of text and determining the meaning or thought behind the songs. The focus of the paper is on Emotion Recognition from music lyrics through text processing. The fundamental concepts in emotion analysis from music lyrics (text) are described. An overview of emotion models, music features, and data sets used in different studies is given. The features of ANEW, a widely used corpus in emotion analysis, are highlighted and related to the music emotion analysis. A comprehensive review of some of the prominent work in emotion analysis from music lyrics is also included.
\end{abstract}

Keywords: Music lyrics, Emotion Analysis, Affective Norms, Music emotion models. 
ABSTRACTS

\title{
A Deep Neural Network Model With Multihop Self- Attention mechanism for Topic Segmentation of Texts
}

\author{
Fayçal Nouar ${ }^{1}$ and Hacene Belhadef ${ }^{2}$ \\ ${ }^{1}$ Management Sciences Department, May 8th 1945 Guelma University, Algeria \\ ${ }^{2}$ NTIC Faculty, University of Constantine 2 - Abdelhamid Mehri, Algeria \\ noufayssalegmail.com
}

\begin{abstract}
Topic segmentation is an important task in the field of natural language processing (NLP), which finds its importance in applications such as information retrieval, text summarization, e-learning. Current neural methods for topic segmentation represent a sentence by a single feature vector that generates single semantic information. However, the dependencies between different parts in a sentence relies on more complex semantic information, which cannot be learned by a single-vector representation. In this paper, we present a deep neural model to capture the multi-aspect semantic information for topic segmentation of texts by multi-hop attention mechanism to address this issue, which named MHOPSA-SEG. At each attention step, the model assigns different weights to words depending on the previous memory weights. Thus, it can capture multiple sentence semantic vector representation. We conduct experiments on four datasets, including written texts and lectures transcripts. And the experimental results show that MHOPSA-SEG outperforms the state-of-the-art models.
\end{abstract}

Keywords: Topic segmentation, BERT, RNN, Highway network, Multihop self-attention, NLP. 
ABSTRACTS

Data Science and Big Data Analytics 
ABSTRACTS

\title{
Big Data Interoperability Framework for Malaysian Public Open Data
}

\author{
Najhan Muhamad Ibrahim ${ }^{1}$, Amir Aatieff Amir Hussin², Khairul Azmi Hassan ${ }^{3}$ and \\ Ciara Breathnach ${ }^{4}$ \\ ${ }^{1}$ Department of Information System, International Islamic University Malaysia, Jalan Gombak, \\ 53100, Selangor \\ ${ }^{2}$ Department of Computer Sciences, International Islamic University Malaysia, Jalan Gombak, \\ 53100, Selangor \\ ${ }^{3}$ Health Research Institutes, University of Limerick, V94 T9PX, Ireland \\ najhan_ibrahimeiium.edu.my, amiraatieffeiium.edu.my, \\ mkazmi@iium.edu.my, Ciara.Breathnach@ul.ie
}

\begin{abstract}
Massive quantities of Malaysia Open Data are available in the public domain such as provided by data.gov.my. However, most of the available datasets are not integrated. Some are unstructured and structured following its source of datasets. Naturally, the datasets cannot interconnect or 'interoperable' with one another, which leads to Big Data (BD) problem. Advances in the database management system and interconnect linked data techniques to connect database systems, provide extraordinary opportunities to create relationships between distributed datasets for a particular objective. Fast-growing in computing technologies, which lead to the digitization, which lead to the capability to query various open datasets. Public Open Data come in varying sources, sizes, and formats. These Big and Small datasets formats pose various integration problems for Information Technology Frameworks. To generate meaningful linked-data to support the purposes of our study the relationship between these disparate datasets needs to be identified and integrated. This paper proposes a BD interoperability framework to integrate Malaysian public health open data. The main goal to enable the potential application with current technologies to extract and discover from Public Open Data. It would reduce the overall cost for healthcare with better prevention mechanism to be placed at the right time. By having a public open big data framework in health, we would predict the pattern of future disease that may take several years to understand.
\end{abstract}

Keywords: Big Data, Public Open Data, Interoperability Framework. 
ABSTRACTS

\title{
The Digital Resources Objects Retrieval: Concepts and Figures
}

\author{
Wafa' Za'al Alma'aitah ${ }^{1,2}$, Abdullah Zawawi Talib ${ }^{2}$, Mohd Azam Osman ${ }^{2}$ \\ ${ }^{1}$ Department of Basic Sciences, The Hashemite University, Zarqa, Jordan \\ ${ }^{2}$ School of Computer Sciences, Universiti Sains Malaysia, 11800 Gelugor, Pulau \\ Pinang, Malaysia \\ Wafaa_maitah@hu.edu.jo, azht@usm.my, azam@usm.my
}

\begin{abstract}
Rapid growth of digital resource objects (DRO) and the valuable contents in such resources have increased the availability of these resources to the users. In attempting to enhance the accessibility of these resources, it is necessary to cater the needs of the users as well as provide search outcomes which are closer to the request. Recently, researchers converted the search path in DRO search from the data retrieval (DR) approach to the information retrieval (IR) approach. Various DRO retrieval systems have been built to facilitate the process of accessing the DRO contents. Thus, such systems need to evaluate their performance effectivity. This paper presents the characteristics of the collections that should be made available in the DRO test collections. It also reviews the computational evaluations and statistical tests used to evaluate the performance of the DRO retrievals.
\end{abstract}

Keywords: digital resource objects, evaluations, test collections, statistical tests 
ABSTRACTS

\title{
A Review of Graph-Based Extractive Text Summarization Models
}

\author{
Abdulkadir Abubakar Bichi ${ }^{1}$, Ruhaidah Samsudin ${ }^{1}$, Rohayanti Hassan ${ }^{1}$ and Khalil \\ Almekhlafi $^{2}$ \\ ${ }^{1}$ School of Computing, Universiti Teknologi Malaysia \\ ${ }^{2}$ University, CBA-Yanbu, 42353, Saudi Arabia \\ engraabubakar@gmail.com, ruhaidah@utm.my, rohayanti@utm.my, \\ drkhalilmekhlafi@gmail.com
}

\begin{abstract}
The amount of text data is continuously increasing both at online and offline storage, that makes is difficult for people to read across and find the desired information within a possible available time. This necessitate the use of technique such as automatic text summarization. A text summary is the briefer form of the original text, in which the principal document message is preserved. Many approaches and algorithms have been proposed for automatic text summarization including; supervised machine learning, clustering, graph-based and lexical chain, among others. This paper presents a review of various graph-based automatic text summarization models.
\end{abstract}

Keywords: Natural Languages Processing, Text Mining, Graph approaches. 
ABSTRACTS

\title{
Review on Emotion Recognition using EEG signals Based on Brain-Computer Interface System
}

\author{
Mona Algarni and Faisal Saeed \\ College of Computer Science and Engineering, Taibah University, Medina, Saudi Arabia \\ mona-algarni@hotmail.com, fsaeed@taibahu.edu.sa
}

\begin{abstract}
Deep learning is closely related to theories of brain development. BrainComputer Interface (BCI) is the latest development in human-computer interaction (HCI). The BCI reads brain signals from different areas of the human brain and translates these signals into commands that can be controlled through the computer applications. BCI technology is effective in the field of human emotions recognition, with high accuracy using EEG signals. When the brain signals are collected and analyzed using deep learning algorithms, it helps in diagnosing diseases and in distinguishing between physical and psychological diseases, which is helpful in making a correct medical decision. The combination of feature selection methods and classification algorithms serves to recognize emotion more accurately from EEG signals. Each of these algorithms has degree of accuracy and unique characteristics. In this paper, we have reviewed and discussed the related studies on BCI technology that are most concerned with classification of emotions through EEG signals. In addition, we have reviewed the methods of collecting signals and feature extraction from EEG datasets. The paper also discusses the main challenges faced in emotion recognition using EEG. We have reviewed several recent studies are classified based on the techniques used in the emotion recognition process. The results show a clear increase in research related to emotion recognition as an important area of investigation, and a diversity of techniques being used to extract and classify features. After discussing the challenges, we found that given the state of technological development, the interconnection between technology and medicine will generate a tremendous volume of applied solutions in future, contributing to the development of research in health informatics systems. A comparison of the recent studies in this field has been conducted, and we deduce the wide variety of techniques used to detect emotion and the increasingly accurate results.
\end{abstract}

Keywords: Brain-Computer Interface, Classification Methods, Deep Learning, EEG, Electroencephalography, Emotion Recognition, Feature Extraction. 
ABSTRACTS

\title{
A New Multi-Resource Deadlock Detection Algorithm Using Directed Graph Requests in Distributed Database Systems
}

\author{
Khalid Al-Hussaini ${ }^{1}$, Nabeel A. Al-Amdi ${ }^{2}$ and Fuaad Hasan Abdulrazzak ${ }^{2}$ \\ ${ }^{1}$ Faculty of Computer Science and Information Systems, Thamar University, Genius University \\ for Sciences and Technology, Yemen \\ ${ }^{2}$ Faculty of Computer Science and Information Systems/ Thamar University, Yemen \\ khalid00alhussaini@gmail.com, \\ nabeel.alamdi2019@gmail.com, fuaad.Abdulrazzak@gmail.com
}

\begin{abstract}
In distributed system, a single database is spread physically across computers in multiple locations called distributed database. One of the most serious problems in distributed database is deadlock. The deadlock is a state of the system in which transactions are waiting for one another indefinitely. This paper presents a new algorithm to detect multi resource deadlocks using directed graph. The proposed algorithm is development over the algorithms by Brain M. Johnston and Himanshi Grover. In previous algorithms, there is no criteria to decide the transaction, which needs to be aborted early to reduce the repeated time of detections. It makes this decision using incoming and out coming requests of transactions in graph as criteria to decide such transaction to detected and aborted early. It ensures that only one transaction will detect the deadlock cycle. All true deadlocks are detected in finite time and no false and undetected deadlocks are reported.
\end{abstract}

Keywords: Distributed Database, Multi-Resource Deadlock, Deadlock Detection, Wait-For-Graph. 
ABSTRACTS

\title{
Big Data Analytics Model for Preventing the Spread of COVID-19 During Hajj Using the Proposed Smart Hajj Application
}

\author{
Ibtehal Nafea \\ Taibah University, AlMadinah Almonwara, Saudi Arabia \\ inafea@taibahu.edu.sa
}

\begin{abstract}
Following the declaration of COVID-19 as a global disaster, one of the affected events is the Hajj. Ideally, the desire by the faithful to observe their religious practices calls for collaborative efforts. Ideally, the Kingdom of Saudi Arabia can adopt smart technology in facilitating the fight against the spread of the disease. After declaring Covid 19 as a global pandemic, new challenges for the healthcare sector in Hajj have emerged. Hajj represents a major challenge to the Saudi Ministry of Health $(\mathrm{MOH})$ in addressing early detection of infection and controlling it at large gatherings of people. As seen in countries such as Australia, Bahrain, and China, smart technology enhances contact tracing, isolation, and even monitoring. Through the proposed Smart Hajj application, pilgrimage will be facilitated in a manner allowing the collection of data on the people. Ideally, the application will be applied from the start of the trip to the end. As such, the application allows the generation a solution from different data resources for the prevention of COVID-19 thus enhancing the pilgrimage experience.
\end{abstract}

Keywords: Hajj, Big data, COVID19, Cloud computing, Saudi Arabia 
ABSTRACTS

\title{
Financial Time Series Forecasting Using Prophet
}

\author{
Umi Kalsom Yusof ${ }^{1}$, Mohd Nor Akmal Khalid ${ }^{2}$, Abir Hussain ${ }^{3}$ and Haziqah \\ Shamsudin ${ }^{1}$ \\ Umi Kalsom Yusof 1[0000-0003-4358-7430], Mohd Nor Akmal Khalid 2[0000-0002-7909-8869], Abir \\ Hussain $^{3[0000-0001-8413-0045]}$ and Haziqah Shamsudin ${ }^{1[0000-0003-3199-5752] ~}$ \\ ${ }^{1}$ School of Computer Sciences, USM, 11800 Georgetown, Pulau Pinang, Malaysia \\ ${ }^{2}$ School of Information Science, JAIST, Nomi, 923-1211, Japan \\ ${ }^{3}$ Department of Computer Science, LJMU, Liverpool, L3 3AF, United Kingdom \\ umiyusof@usm.my, akmal@jaist.ac.jp, A.Hussain@ljmu.ac.uk, \\ haziqahshamsudin@student.usm.my
}

\begin{abstract}
Forecasting the financial time series had been a difficult endeavor for both academia and businesses. Advances of the financial time series forecasting had moved from traditional techniques to automated and intelligent techniques that based on machine learning and deep learning. However, many methods of automatic forecasting have been tailored to the specific nature of the time series. As such, a recently introduced Prophet model, which is based on time series decomposition, is adopted with variants of its input parameters and applied to six different financial time series data sets obtained from Standard \& Poor's 500 index (SP500), Dow Jones Industrial Average index (DJIA), China Securities Index (CSI300), Malaysia's stock market of Kuala Lumpur Composite Index (KLCI), Hong Kong Hang Seng 300 index (HS300) and Tokyo's stock market of Nihon Keizai Shinbun index (Nikkei). The results of the time series forecasting show that the Prophet model is competitive in modeling the actual market movement by simply adopting appropriate parameters where the measure of Mean Absolute Percentage Errors (MAPE) was 6\% at most. In addition, the errors of the forecasting result are also comparable to the results of much more complex forecasting models from the literature.
\end{abstract}

Keywords: Financial Time Series, Prophet, Time Series Forecasting. 
ABSTRACTS

\title{
Facial Recognition to identify Emotions; An application of Deep Learning
}

\author{
Kenza Belhouchette \\ Research Laboratory on Computer Science's Complex Systems , Larbi Ben M'Hidi \\ University,Algeria \\ belhouchet_kenza@hotmail.com
}

\begin{abstract}
Deep learning is an approach that is not recent. But its use in the field of emotion recognition is a very important and very recent subject. Because of its power in classification. In this work we used convolutional neural networks for based emotions recognition. (joy, sadness, anger, disgust, surprise, fear and neutral). Our proposed work is an intelligent system of emotion recognition with mathematical foundations explanation of convolutional neural networks. To evaluate our recognition system we used two evaluation metrics which are : The rate of good classification (tbcs) and Error rate. The recognition rate achieved is very satisfactory. Indeed our recognition system was able to recognize almost more than $90 \%$ of emotions.
\end{abstract}

Keywords: Emotion, Convolutional neural network, facial expression. 
ABSTRACTS

\title{
Text-based Analysis to Detect Figure Plagiarism
}

\author{
Taiseer Abdalla Elfadil Eisa ${ }^{1}$, Naomie Salim ${ }^{2}$ and Salha Alzahrani ${ }^{3}$ \\ ${ }^{1}$ College of Science and Arts- Girls Section, King Khalid University Mahayil, Asir, Saudi, \\ Arabia. \\ ${ }^{2}$ Faculty of Computer Science and Information Systems, Universiti Teknologi Malaysia, 1310, \\ Skudai, Johor, Malaysia. \\ ${ }^{3}$ Dept. of Comp. Science, Taif University, Taif, Saudi Arabia \\ Teisa@kku.edu.sa
}

\begin{abstract}
Plagiarism, the process of copying someone else's text or data without due recognition of the source is a serious academic offence. Many techniques have been proposed for detecting plagiarism in texts but only few techniques exist for detecting figure plagiarism. The main problem associated with existing techniques is that they are not applicable to non-textual elements of figures in research publications. This paper addresses the problem of figure plagiarism in scientific articles and proposes solutions to detect cases where an exact copy or modified figure retains the essential data in the original figure. In this paper, we proposed a deep figure analysis to detect all types of possible figure plagiarism ranging from simple copy and paste to plagiarism of strong modification to the content of the figure source. Unlike existing figure plagiarism detection methods, which compare between figures based on surface features. The proposed method represents each component of a figure and provides information about the text inside its component and the relationships with other component(s) to capture the meaning of the figure. using component-based comparison, and an improvement over existing methods which cannot extract enough information from figures to detect plagiarism. The results obtained by the proposed method are considered as one of the interesting research solutions for figure plagiarism.
\end{abstract}

Keywords: Plagiarism detection, Figure plagiarism detection, Similarity detection, Image plagiarism detection, Semantic similarity, figures text detection, figure text analysis 
ABSTRACTS

\title{
A Virtual Exploration of Al-Masjid Al-Nabawi Using Leap Motion Controller
}

\author{
Slim Kammoun ${ }^{1,3}$ Hamza Ghandorh ${ }^{2}$ \\ ${ }^{1}$ Information System Department, Taibah University, Madina; Saudia Arabia \\ ${ }^{2}$ Computer Science Department, Taibah University, Madina, Saudi Arabia \\ ${ }^{3}$ Research Laboratory of Technologies of Information and Communication \& Electrical \\ Engineering, University of Tunis, Tunisia \\ \{Skammoun, hghandor\} @taibahu.edu.
}

\begin{abstract}
Religious tourism is getting popularity and more and more people wants to visit the religious places. Al-Masjid Al-Nabawi holds a historical priority for 1.5 billion visitors with different languages, cultures, and ethnicity. As part of the Saudi leadership to govern the affairs of Al-Masjid Al-Nabawi, many expansions have been plannedlperformed to provide better services for Saudi Arabia's guests and visitors around the years. Large and ongoing expansions of the holy sites in Saudi Arabia may impact the ease of mobility for own guests especially in the time of high seasons (i.e., Haij and Omrah times). Virtual Reality technology started to play a critical role in the tourism industry by virtually exposing users to a certain place. The primary purpose of this paper is to visualize the Al-Masjid Al-Nabawi site in an interactive style and ease of use for the guests. The interaction with the 3D environment is ensured via the Leap motion Controller in a very simple way without need to special learning. We designed and implemented a virtual reality-based touring guide prototype, and an initial user validation was conducted. The results are promising for future investigation.
\end{abstract}

Keywords: Virtual Reality; Leap Motion Controller; Unreal Engine, Al-Masjid Al Nabawi, Toursim 
ABSTRACTS

\title{
Comparison of Data Analytic Techniques for a Spatial Opinion Mining in Literary Works: A Review Paper
}

\author{
Sea Yun Ying ${ }^{1}$, Pantea Keikhosrokiani ${ }^{1}$ and Moussa Pourya Asl ${ }^{2}$ \\ ${ }^{1}$ School of Computer Sciences, Universiti Sains Malaysia, 11800 Minden, Penang, Malaysia \\ ${ }^{2}$ School of Humanities, Universiti Sains Malaysia, 11800 Minden, Penang, Malaysia \\ pantea@usm.my
}

\begin{abstract}
Opinion mining is the use of analytic methods to extract subjective information. A study was conducted to apply spatial opinion mining in literary works to examine the writers' opinions about how matters of space and place are experienced. For this reason, this paper conducts a review study to identify and compare different analytical techniques for opinion mining in fictional writings. This review study focused on sentiment analysis and topic modeling as two main techniques for spatial opinion mining in literary works. The comparison results are reported and the limitations of different techniques are mentioned. The results of this study can assist researchers in the field of opinion and text mining.
\end{abstract}

Keywords: Big Data Analytics; Opinion Mining, Text Mining, Sentiment Analysis, Topic Modeling, literary works. 
ABSTRACTS

\title{
Open Data in Prediction using Machine Learning: A Systematic Review
}

\author{
Norismiza Ismail ${ }^{1,2}$ and Umi Kalsom Yusof ${ }^{1}$ \\ ${ }^{1}$ School of Computer Sciences, Universiti Sains Malaysia, 11800 Penang, Malaysia \\ 2 Digital Management and Development Centre, Universiti Malaysia Perlis, 02600 Arau, \\ Perlis, Malaysia \\ norismiza@unimap.edu.my, norismizadstudent.usm.my, umiyu- \\ sof@usm.my
}

\begin{abstract}
The determinants of open data (OD) in prediction using machine learning (ML) have been discussed in this study, which is done by reviewing current research scenario. As open government data (OGD) and social networking services (SNSs) have grown rapidly, OD is considered as the most significant trend for users to enhance their decision-making process. The purpose of the study was to identify the proliferation of OD in ML approaches in generating decisions through a systematic literature review (SLR) and mapping the outcomes in trends. In this systematic mapping study (SMS), the articles published between 2011 and 2020 in major online scientific databases, including IEEE Xplore, Scopus, ACM, Science Direct and Ebscohost were identified and analyzed. A total of 576 articles were found but only 72 articles were included after several selection process according to SLR. The results were presented and mapped based on the designed research questions (RQs). In addition, awareness of the current trend in the OD setting can contribute to the real impact on the computing society by providing the latest development and the need for future research, especially for those dealing with the OD and ML revolution.
\end{abstract}

Keywords: systematic literature review, systematic mapping study, open data, prediction, machine learning 
ABSTRACTS

\title{
Big Data Analytics Based Model for Red Chili Agirculture in Indonesia
}

\author{
Junita Juwita Siregar ${ }^{10000-0002-5233-3101]}$ and Arif Imam Suroso ${ }^{2}$ \\ ${ }^{1}$ Computer Science Department, School of Computer Science, Bina Nusantara University, \\ Jakarta Indonesia, 11480. \\ ${ }^{2}$ School of Business, IPB University, Bogor West Java, Indonesia \\ juwita_siregar@binus.ac.id, arifimamsuroso@apps.ibp.ac.id
}

\begin{abstract}
Horticulture chili plants are a food crop with significant role in the Indonesian macroeconomy. Chili production increases in certain months, which is followed by domestic demand for chili. As production centers for chili plants are concentrated in several provinces, production sometimes is unable to meet consumption demands. This results in a severe increase in chili prices in certain months. The disparity in chili prices in some areas can impact farmers. The purpose of this paper is to develop a model that can be used for the implementation of big data analytics (BDA) in the red chili horticultural agro-industry. Big Data Analytics techniques are applied to develop a predictive model. The research method used a qualitative content analysis approach. The results of this study is a proposed model based on BDA that is applicable to agribusiness of red chili plants in Indonesia. Thus, it could be used in making decisions for farmers to plan optimal chili production schedule and plan logistics and distribution chains to several regions so that farmers can reduce the production cost and increase profit for the farmer.
\end{abstract}

Keywords: Machine learning. Big Data Analytics. Red Chili Agriculture. 
ABSTRACTS

\title{
A Fusion-Based Feature Selection Framework for Microarray Data Classification
}

\author{
Talal Almutiri ${ }^{1}$, Faisal Saeed ${ }^{2}$, Manar Alassaf $^{3}$, Essa Abdullah Hezzam² \\ ${ }^{1}$ Department of Information Systems, Faculty of Computing and Information Technology, \\ King Abdulaziz University, Jeddah, Saudi Arabia \\ ${ }^{2}$ Department of Information Systems, College of Computer Science and Engineering, Taibah \\ University, Medina, Saudi Arabia \\ ${ }^{3}$ Department of Computer Science, College of Computer, Qassim University, Buraydah, Saudi \\ Arabia \\ Almutiri.Talal@hotmail.com, fsaeedetaibahu.edu.sa, \\ ehezzam@taibahu.edu.sa, a.manar@qu.edu.sa
}

\begin{abstract}
Gene expression profiling uses microarray techniques to discover patterns of genes when they are expressed. This helps to draw a picture of how the cell performs its function and determines whether there are any mutations. However, microarrays generate a huge amount of data which causes a computational cost and is time-consuming in the analysis process. Feature selection is one of the solutions for reducing the dimensionality of microarray datasets by choosing important genes and eliminating redundant and irrelevant features. In this study, a fusion-based feature selection framework was proposed that aims to apply multiple feature selection methods and combine them using ensemble methods. The framework consists of three layers; in the first layer, there are three feature selection methods that worked independently for ranking genes and assigned a score for each gene. In the second layer, a threshold is used to filter each gene according to their calculated scores. In the last layer, the final decision about which genes are important is made based on one of the decision voting strategies, either majority or consensus. The proposed framework presented an improvement in terms of classification accuracy and dimensionality reduction when compared with other previous methods.
\end{abstract}

Keywords: Cancer Classification, Gene Expression, Feature Selection, Fusion, Microarray Data 
ABSTRACTS

\title{
An Approach based Natural Language Processing for DNA Sequences Encoding using the Global Vectors for Word Representation
}

\author{
Brahim Matougui ${ }^{1,2}$, Hacene Belhadef ${ }^{1}$ and Ilham Kitouni ${ }^{1}$ \\ ${ }^{1}$ University of Constantine 2- Abedelhamid Mehri, 25016 Algeria. \\ ${ }^{2}$ National Center for Biotechnology Research, Constantine, 25016, Algeria \\ brahim.matougui@univ-constantine2.dz
}

\begin{abstract}
DNA sequence has several representations; one of them is to split it into k-mers components. In this work, we explore the high similarity between natural language and "genomic sequence language" which are both character-based languages, to represent DNA sequences. In this representation, we processed a DNA sequence as a set of overlapping word embeddings using the Global Vectors representation. In Natural language processing context, we can consider k-mers as words. The embedding representation of k-mers helped to overcome the curse of dimensionality, which is one of the main issues of traditional methods that encode k-mers occurrence as one hot vector. Experiments on the first Critical Assessment of Metagenome Interpretation (CAMI) dataset demonstrated that our method is an efficient way to cluster metagenomics reads and predict their taxonomy. This method could be used as first step for metagenomics downstream analysis.
\end{abstract}

Keywords: Global Vectors representation, word embeddings, DNA sequence representation, Natural Language Processing. 


\title{
ABSTRACTS
}

\section{Short-term CO2 Emissions Forecasting Using Multi- Variable Grey Model and Artificial Bee Colony (ABC) Algorithm Approach}

\author{
Ani Shabri ${ }^{1}$, Ruhaidah Samsudin ${ }^{2}$, Essa Abdullah Hezzam ${ }^{3}$ \\ ${ }^{1}$ Mathematics Department, Faculty of Science, Universiti Teknologi Malaysia, 81310, Johor, \\ Malaysia \\ ${ }^{2}$ Faculty of Engineering, Universiti Teknologi Malaysia, Johor Bahru, Malaysia \\ ${ }^{3}$ Department of Information Systems, College of Computer Science and Engineering, Taibah \\ University, Medina, Saudi Arabia \\ Alani@utm.my, ruhaidah@utm.my, ehezzam@taibahu.edu.sa
}

\begin{abstract}
Carbon dioxide (CO2) emissions is one of the recent global issues where the negative influence and effect on the environment is high. Enhancing the degree of awareness among public and concerned authorities and developing forecasting methods and techniques form a vital solution to this issue. The aim of this research is to enhance the forecasting efficiency of the traditional $\mathrm{GM}(1, \mathrm{~N})$ model by proposing and modifying background values of $\operatorname{GM}(1, N)$ using a new algorithms. This paper presents the Artificial Bee Colony (ABC) to select the optimal weight of background values for a traditional GM(1,N) model. The data of $\mathrm{CO} 2$ emissions, GDP per capita, the amount invested in Malaysia, population, total energy consumption and number of registered motor vehicles during the period from 2000 to 2016 is used to verify the applicability and effectiveness of the model. The numerical example results indicate that the new model is performing well compared to the traditional $\mathrm{GM}(1, \mathrm{~N})$ model.
\end{abstract}

Keywords: multi-variable grey model, Artificial Bee Colony, Carbon dioxide emissions forecasting. 
ABSTRACTS

IoT and Intelligent Communication Systems 
ABSTRACTS

\title{
A Reliable Single Prediction Data Reduction Approach for WSNs based on Kalman Filter
}

\author{
Zaid Yemeni ${ }^{1}$, Haibin Wang ${ }^{1}$, Waleed M.Ismael ${ }^{1}$, Younis Ibrahim ${ }^{1}$, and Peng Li $^{2}$ \\ ${ }^{1}$ Hohai University, Changzhou Campus, Changzhou, Jiangsu, China \\ ${ }^{2}$ Shanghai Xieji Technology Company, Shanghai, China \\ wanghaibin@hhuc.edu.cn, yemenizaidehhu.cn, \\ waleed.m@hhu.edu.cn, younisibrahim@hhu.edu.cn, lipeng0105@126.com
}

\begin{abstract}
Wireless sensor networks (WSNs) are critically resource-constrained due to wireless sensor nodes' tiny memory, low processing unit, power limitation, and narrow communication bandwidth. The data reduction technique is one of the most widely used techniques to minimize the transmitted data over the entire network and overcome the limitations mentioned above. In this paper, a reliable single prediction data reduction approach is proposed for WSNs. The proposed approach is built on two phases: the Data Reduction (DR) Phase and Data Prediction (DP) Phase. In the first phase (DR), the proposed approach aims at minimizing the total data transmission using two techniques, Data Equality (DE) and Data Change Detection (DCD). In the second phase (DP), the non-transmitted data are predicted on the sink node utilizing the wellknown Kalman filter. The obtained results demonstrate that the proposed approach is efficient and effective in data reduction and data reliability.
\end{abstract}

Keywords: Data Reduction, Reliability, WSN, Data Prediction. 
ABSTRACTS

\title{
A Real-Time Groundwater Level Monitoring System Based on WSN, Taiz, Yemen
}

\author{
Asma'a K. Akershi, Ziad S. Arobieh, Reayidh A. Ahmed \\ Faculty of Engineering and Information Technology, Taiz University, Taiz, Yemen \\ AsmaaAlkershi123@gmail.com, Ziadrh@yahoo.com, \\ ReayidhalAlwaney@gmail.com
}

\begin{abstract}
Rise in the population, climate instability, and unregulated groundwater mining threaten the preservation of aquifers worldwide. Effective and data-driven control of groundwater supply is essential for sustaining critical water-dependent functions. Recently, the water crisis in Yemen has become a problem threatening the lives of many residents. This is due to the manager's lack of cost-effective, scalable, and reliable groundwater monitoring systems needed to gather vital groundwater data. In this study, we developed a fully automated real-time groundwater level system for data collection and visualization based on wireless sensor network (WSN). The study was applied in Taiz City, Yemen. The steps used to develop the system, including dividing the study area into different zones, and each zone uses a local base-station to collect the data from the sensors deployed in the same zone. All local base-station send the data to the master base station located remotely. The master bases station collects the data and sends it to the web-servers that are used to save and visualize the data permanently. The proposed system was designed and evaluated using the Castalia Omnet++ simulator and the system performance investigated based on network bandwidth (number of packets sent) and the sensors' power consumption. As expected, the results show that using local base station reduces the number of packets sent to the remote master station, this help extending the life of the water level sensors used.
\end{abstract}

Keywords: Groundwater wells, WSN, water level, Taiz, Castalia Omnet++. 


\title{
Design and Simulation of Multiband Circular Microstrip Patch Antenna with CSRR for WLAN and WiMAX Applications
}

\author{
Abdulguddoos S. A. Gaid ${ }^{1}$, Amer A. Sallam², Mohamed H. M. Qasem ${ }^{1}$, Maged S. G. \\ Abbas $^{1}$ and Amjad M. H. Aoun ${ }^{1}$ \\ ${ }^{1}$ Dept. of Communication and Computer Engineering, Faculty of Engineering, Taiz University, \\ Taiz, Yemen \\ ${ }^{2}$ Dept. of Computer Networks and Distributed Systems, Faculty of Engineering, Taiz \\ University, Taiz, Yemen \\ asry2000@hotmail.com, amer.sallametaiz.edu.ye, \\ mohamedqasem270320@gmail.com, magood.9.1111@gmail.com, \\ amjad770497211@gmail.com
}

\begin{abstract}
In this work, a single-feed circular microstrip patch antenna was proposed, designed and optimized for WLAN and WiMAX applications. The proposed antenna was printed on FR-4 dielectric substrate material with a relative permittivity $\left(\varepsilon_{\mathrm{r}}\right)$ of 4.4 , a loss tangent $(\tan \delta)$ of 0.02 and a height $(\mathrm{h})$ of $1.6 \mathrm{~mm}$. The characteristics of the proposed antenna were improved by etching two square-shaped Complementary Split Ring Resonators (CSRR) on a partial ground plane and one square-shaped CSRR on the main circular patch radiator. Radius of the circular patch is $12 \mathrm{~mm}$ and the overall size of the structure is being $50 \times 40 \times 1.6 \mathrm{~mm}^{3}$. The proposed design is a multiband antenna resonating at four resonant frequencies and operating at two frequency bands. The antenna covers the frequency band extending from $2.2995 \mathrm{GHz}$ to $2.84 \mathrm{GHz}$ with a bandwidth of $540 \mathrm{MHz}$ and the frequency band extending from $3.36 \mathrm{GHz}$ to 5.9273 $\mathrm{GHz}$ with a bandwidth of $2.5673 \mathrm{GHz}$. The antenna resonates at $2.52 \mathrm{GHz}, 3.48 \mathrm{GHz}$, $5 \mathrm{GHz}$ and $5.52 \mathrm{GHz}$ achieving peak gains of $2.52 \mathrm{~dB}, 2.5416 \mathrm{~dB}, 3.014 \mathrm{~dB}$ and $4 \mathrm{~dB}$ respectively. The proposed antenna may be applied for WLAN/ WiMAX applications. The design and simulation were performed using the High Frequency Simulator Software (HFSS).
\end{abstract}

Keywords: Compact Multiband, Microstrip Patch Antenna, Complementary Split Ring Resonators (CSRR), WLAN/WiMAX Applications, Defected Ground Structure (DGS). 
ABSTRACTS

\title{
Reference Architectures for the IoT: A Survey
}

\author{
Raghdah Saemaldahr, Bijayita Thapa, Kristopher Maikoo, and Eduardo B. Fernandez \\ Florida Atlantic University, Boca Raton FL 33431, USA \\ rsaemaldahr2018@fau.edu; bthapalfau.eu; \\ kmaikoo2019@fau.edu; fernandedfau.edu
}

\begin{abstract}
The Internet of Things (IoT) has become one of the essential technologies of the past few decades. Its popularity has increased based on its importance and application. It offers a solution to many issues affecting humanity in vital fields. IoT uses sensors and actuators to perform operations that complement higher-level applications. Building IoT applications requires using an architectural structure to support the application functions. Architectural modeling using patterns and Reference Architectures (RAs) applies abstraction, which helps in reducing the complexity of IoT systems. Because of this complexity, security is an important problem in IoT applications. An RA is the basis to build a security reference architecture (SRA). We survey the most important IoT RAs, showing their features and drawbacks and compare them accordingly. From the survey we find that the RA representations are imprecise and not very detailed. We define the features for a better RA that will be used as a basis for a SRA after enumerating its possible vulnerabilities and threats and then placing appropriate defenses.
\end{abstract}

Keywords: Internet of Things, Reference Architecture, Security Patterns, Security. 
ABSTRACTS

\title{
A Circular Multiband Microstrip Patch Antenna with DGS for WLAN/WiMAX/Bluetooth/UMTS/LTE
}

\author{
Abdulguddoos S. A. Gaid ${ }^{1}$, Amer A. Sallam², Mohamed H. M. Qasem¹, \\ Maged S. G. Abbas ${ }^{1}$, and Amjad M. H. Aoun ${ }^{1}$ \\ ${ }^{1}$ Dept. of Communication and Computer Engineering, ${ }^{2}$ Dept. of Computer Networks and \\ Distributed Systems \\ Faculty of Engineering, Taiz University, Taiz, Yemen, Faculty of Engineering, Taiz \\ University, Taiz, Yemen \\ asry2000@hotmail.com, amer.sallametaiz.edu.ye, \\ mohamedqasem270320@gmail.com, magood.9.1111@gmail.com, \\ amjad770497211@gmail.com
}

\begin{abstract}
This paper proposes a circular multiband, wideband antenna for wireless applications such as WLAN, WiMAX, LTE, Bluetooth, UMTS and suitable for satellite $\mathrm{C}$-band application. Bandwidth enhancement is realized by etching two single squareshaped complementary ring resonators (CSRRs) on the partial ground and three thin rectangular slots on the main circular radiator. The radiating patch is printed on a flame resistant-4 epoxy (FR4) dielectric substrate with an overall size of $70 \mathrm{~mm} \times 70 \mathrm{~mm} \times$ $3.6 \mathrm{~mm}$, a dielectric constant of 4.4 and a loss tangent of 0.02 . The presented antenna operates at (1.9-2.65) GHz band centered at $2.24 \mathrm{GHz}$ and at (3.34-7.84) GHz band with four resonant frequencies $(3.43 \mathrm{GHz}, 4.36 \mathrm{GHz}, 5.56 \mathrm{GHz}$ and $7.51 \mathrm{GHz})$. The total bandwidth achieved is $5.25 \mathrm{GHz}$ and the peak gains achieved at resonant frequencies are $2.7 \mathrm{~dB}, 5.72 \mathrm{~dB}, 4.55 \mathrm{~dB}, 3.85 \mathrm{~dB}$ and $5.8 \mathrm{~dB}$ respectively. This design and simulation are carried out using the High structural Frequency Simulator Software package.
\end{abstract}

Keywords: Wideband Antennas, Circular Microstrip Patch Antenna, Defected Ground Structures (DGS), complementary split ring resonator (CSRR), Partial Ground. 
ABSTRACTS

\title{
Anomaly Intrusion Detection Systems in IOT using Deep Learning Techniques: A Survey
}

\author{
Muaadh. A. Alsoufi ${ }^{1}$, Shukor Razak ${ }^{1}$, Maheyzah Md Siraj ${ }^{1}$, Abdulalem Ali ${ }^{1}$, \\ Maged Nasser ${ }^{1}$, Salah $\mathrm{Abdo}^{2}$ \\ ${ }^{1}$ School of Computing, Universiti Teknologi Malaysia, Johor Bahru, Johor 81310, Malaysia \\ ${ }^{2}$ Department of Communication and computer Engineering, Faculty of Engineering, UPM, \\ Selangor, 43300, Malaysia.

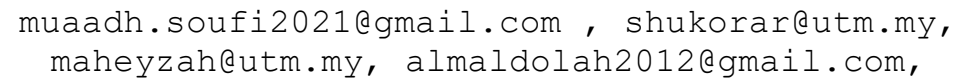

\begin{abstract}
Security has a major role to play in the utilization and operations of the internet of things (IoT). Several studies have explored anomaly intrusion detection and its utilization in a variety of applications. Building an effective anomaly intrusion detection system requires researchers and developers to comprehend the complex structure from noisy data, identify the dynamic anomaly patterns, and detect anomalies while lacking sufficient labels. Consequently, improving the performance of anomaly detection requires the use of advanced deep learning techniques instead of traditional shallow learning approaches. The large number of devices connected to IoT which massively generate a large amount of data require large computation as well. This study presents a survey on anomaly intrusion detection using deep learning approaches with emphasis on resource-constrained devices used in real-world problems in the realm of IoT. The findings from the reviewed studies showed that deep learning is superior to detect anomaly in terms of high detection accuracy and false alarm rate. However, it is highly recommended to conduct further studies using deep learning techniques for robust IDS.
\end{abstract}

Keywords: Anomaly intrusion detection, Internet of things (IoT), Security, Deep Learning, resource constrained. 
ABSTRACTS

\title{
Security and Threats in The Internet of Things Based Smart Home
}

\author{
Nor Fatimah Awang ${ }^{1}$, Ahmad Fudhail Iyad Mohd Zainudin ${ }^{1}$, Syahaneim Marzuki ${ }^{1}$ \\ Syed Nasir Alsagoff ${ }^{1}$, Taniza Tajuddin ${ }^{2}$, Ahmad Dahari Jarno ${ }^{3}$ \\ ${ }^{1}$ National Defense University of Malaysia, Kuala Lumpur, Malaysia \\ ${ }^{2}$ Universiti Teknologi MARA (UiTM) Kedah, Kedah, Malaysia \\ ${ }^{3}$ Cyber Security Malaysia, Kuala Lumpur, Malaysia \\ norfatimah@upnm.edu.my, ahmadfudhail423@gmail.my, \\ syahaneim@upnm.edu.my, syednasir@upnm.edu.my, \\ taniza@uitm.edu.my, dahari@cybersecurity.my
}

\begin{abstract}
The Internet of Things (IoT) carries the meaning of a developing prototype that focuses on the connection between devices, objects, sensors and users. Using IoT, the method of communications and interactions changes in form of 'Human to Thing' to 'Thing to Thing'. IoT technology is rapidly evolving and spreading its technology influence across various domains including healthcare (personal and enterprise), smart campuses, smart homes, smart mobility, and Industry 4.0. As a result, a lot of IoT devices are being used as a crucial component as one of human made high-tech butlers of everyday lifestyle. This paper focuses on the IoT security threats and vulnerabilities that shall be considered in the implementation of IoT ecosystem by conducting a research in the area of smart home environment. The current security threats and vulnerabilities are being discussed in the contexts of the IoT architecture layers, which is the application layer, network layer and physical layer are also presented. This paper also provides an analysis of the possible threats and proposes possible solutions for improving the security of the IoT smart home, that may influence as beneficial factors that are able to promote a secured eco-system of IoT devices in smart home operational environment.
\end{abstract}

Keywords: Internet of Things, Smart Home, Security Threat. 
ABSTRACTS

\title{
Simulation and Control of Industrial Composition Process over Wired and Wireless Networks
}

\author{
Hakim Qaid Abdullah Abdulrab, Fawnizu Azmadi Hussin, Panneer Selvam Arun, \\ Azlan Awang ${ }^{1}$ and Idris Ismail \\ Dept. of Electrical and Electronic Engineering, Univeriti Teknologi PETRONAS, 32610, \\ Perak, Malaysia \\ \{hakim_19001004, fawnizu, arun_18003272, azlanawang, \\ idrisismail\} eutp.edu.my
}

\begin{abstract}
Recently, the wireless technology has played a very important role in the industrial world. WirelessHART and ISA100.11a are the two most standards used in the industry so far. On the other hand, the wired networks are dominating the industrial sectors due to their significant transmission reliability and high security. However, the wired networks require higher maintenance cost and do not support network reconfiguration. In this paper, we will study the performance of a composition control over wired and wireless network. The proposed industrial composition control system in this paper is represented by a three-tank system whose outlet stream concentration is controlled using a concentrated stream of control reagent comprising water and solute. The objective is to design a PID controller using Closed-loop Ziegler Nichols tuning method for the composition control system and further improve the performance of the system by fine-turning the PID parameters. The same parameters of PID controller, that are used to control the system through wired network, are taken and implemented on wireless network using MATLAB Simulink with the aid of TrueTime toolbox. The behavior of both systems is then compared and analyzed.
\end{abstract}

Keywords: Wireless Networked Control Systems (WNCS), WirelessHART, ISA100, TrueTime, Process Control Systems. 
ABSTRACTS

\title{
Performance Degradation of Multi-class Classification Model due to Continuous Evolving Data Streams
}

\author{
Abdul Sattar Palli ${ }^{1}$, Jafreezal Jaafar ${ }^{2}$, Manzoor Ahmed Hashmani ${ }^{3}$ \\ Department of Computer and Information Sciences, Universiti Teknologi PETRONAS, Tronoh \\ 32610, Perak, Malaysia \\ abdulsattarpalliegmail.com
}

\begin{abstract}
Online machine learning plays a pivotal role in the 4th Industrial Revolution (IR 4.0). IR 4.0 requires real-time data analysis (classification or prediction) using the streaming data. However, mostly the data streams contain nonstationary (variability features) characteristics, such as concept drift and class imbalance. The issues of concept drift and class imbalance adversely affects the accuracy of the classification models. The classification accuracy is even more affected when these issues arrived at the same time (joint problem). Some efforts have been made in the literature to cope with the joint problem of class imbalance and concept drift in online learning, but the existing solutions are limited to binary class classification and these solutions do not work for multi-class classification. Besides, the literature doesn't mention the exact correlation between the critical factors of concept drift and class imbalance. Also, the tuning parameters of the multi-class classification models, which can help in improving the classification accuracy, are unknown. However, to resolve the joint problem of concept drift and class imbalance in online multi-class classification models, it is essential to determine the exact correlation and find the tuning parameters, which could be helpful to provide a more dynamic approach for avoiding the performance degradation of the multi-class classification models. Therefore, to resolve this issue, this study aims to determine the correlation between the concept drift and class imbalance, identify the tuning parameters of multi-class classification models and propose a dynamic solution based on these findings. Our proposed dynamic approach could be effectively utilized in various online machine learning based (real-time) streams analysis, which is desirable for IR 4.0.
\end{abstract}

Keywords: Adaptive Framework; Concept Drift; Class Imbalance; Non-stationary, Multi-class Classification 
ABSTRACTS

\title{
Compact Wide-Bandwidth Microstrip Antenna for Millimeter Wave Applications
}

\author{
Osaid Abdulrahman Saeed, Moheeb Ali Ameer, Mansour Noman Ghaleb
}

Dept. of Communication and Computer Engineering, Faculty of Engineering, Taiz University, Yemen

osaid.ucv2016egmail.com

\begin{abstract}
With the advancement of the world to 5th Generation of wireless networks, due to the great demands for higher data rates and better bandwidth utilization in wireless communication systems. Commercial implementation of 5G in many countries such as China, USA, South Korea, UK, Spain and Switzerland, a space is always there to improve radiation characteristics of Antenna for utilizing the high gain and efficient bandwidth to fulfil $5 \mathrm{G}$ demands. This work provides, compact dual-band microstrip antenna for $5 \mathrm{G}$ wireless devices. The proposed antenna has small size with low profile structure integrated on RT5880 substrate with a thickness of $0.5 \mathrm{~mm}$ and patch dimensions of $1.98 \times 1.32 \mathrm{~mm}^{2}$. This antenna resonates at $64 \mathrm{GHz}$ and $92 \mathrm{GHz}$ of mm-wave spectrum with a maximum gain of $7 \mathrm{dBi}$ and $5.7 \mathrm{dBi}$ respectively. Moreover, the achievable bandwidths of this antenna (under $-10 \mathrm{~dB}$ return loss) are $11.3 \mathrm{GHz}(17.4 \%)$ and $24.87 \mathrm{GHz}(26.8 \%)$ at center frequencies $64 \mathrm{GHz} \& 92 \mathrm{GHz}$ respectively. Furthermore, the peak average gain and total bandwidth exhibited over the two bands are $6.285 \mathrm{dBi}$ and $36.17 \mathrm{GHz}$ respectively. High frequency simulation structure (HFSS) is used to perform the design simulation of the proposed antenna. This antenna is very compact, directive and bandwidth efficient (greater than $5 \%$ of the center frequency).
\end{abstract}

Keywords: 5G Networks, Small Size Antenna, High Gain Antenna, Broadband Antenna, Millimeter Wave, 5G Microstrip Antenna. 
ABSTRACTS

\title{
Dual-Band Rectangular Microstrip Patch Antenna with CSRR for 28/38 GHz Bands Applications
}

\author{
Abdulguddoos S. A. Gaid ${ }^{1}$, Mohamed H. M. Qasem ${ }^{1}$, Amer A. Sallam² and Ebrahim \\ Q. M. Shayea ${ }^{3}$ \\ ${ }^{1}$ Dept. of Communication and Computer Engineering, Faculty of Engineering, Taiz University, \\ Taiz, Yemen \\ ${ }^{2}$ Dept. of Computer Networks and Distributed Systems, Faculty of Engineering, Taiz \\ University, Taiz, Yemen \\ ${ }^{3}$ Operation and Maintenance, Sabafon GSM Mobile Company, Yemen \\ asry2000@hotmail.com, mohamedqasem270320@gmail.com, \\ Amer.sallam@taiz.edu.ye, Ibrahimqaid2016@gmail.com
}

\begin{abstract}
This paper presents a dual-band microstrip patch antenna operating at 28/38 $\mathrm{GHz}$ bands used in $5 \mathrm{G}$ mobile networks. The proposed structure is integrated to a Rogers RT/duroid 5880 substrate material with a relative permittivity of 2.2, height of 0.6 $\mathrm{mm}$ and loss tangent of 0.0009 . To get dual resonance and hence an enhanced bandwidth, two square-shaped Complementary Split Ring Resonators (CSRR) are etched, one on the radiating patch and the other on the ground plane. The structure is compact with an overall size of $7 \mathrm{~mm} \times 7 \mathrm{~mm} \times 0.6 \mathrm{~mm}$. Simulation shows that, the patch is operating at two frequency bands. The first band, centered around $28 \mathrm{GHz}$ has a bandwidth of $1.94 \mathrm{GHz}$ while the second band is $4.8 \mathrm{GHz}$ bandwidth around the $38 \mathrm{GHz}$ resonant frequency. The peak gains obtained are $8 \mathrm{dBi}$ and $8.75 \mathrm{dBi}$ at the resonant frequencies respectively. The designed antenna shows low profile, good gain and bandwidth efficient. These characteristics make the patch suitable for mm-waves $5 \mathrm{G}$ applications. The antenna is designed and simulated using the High Frequency Structure Simulator (HFSS).
\end{abstract}

Keywords: Dual-band, Microstrip patch antenna, Bandwidth enhancement, Complementary Split Ring Resonator (CSRR), mm-w 5G wireless communication. 


\title{
Dual Band Rectangular Microstrip Patch Antenna for 5G Millimeter-Wave Wireless Access and Backhaul Applications
}

\author{
Abdulguddoos S. A. Gaid ${ }^{1}$, Amer A. Sallam², Amjad M. H. Aoun ${ }^{1}$, Ahmed A. A. \\ Saeed $^{1}$, and Osama Y. A. Sae'ed ${ }^{1}$ \\ ${ }^{1}$ Dept. Of Communication and Computer Engineering, Faculty of Engineering \& IT, Taiz \\ University, Yemen \\ ${ }^{2}$ Dept. Of Computer Networks and Distributed Systems, Faculty of Engineering \& IT, Taiz \\ University, Yemen \\ asry2000@hotmail.com, amer.sallametaiz.edu.ye, \\ amjad770497211@gmail.com, ahmedalabsy947@gmail.com, \\ osama.12.12.12cegmail.com
}

\begin{abstract}
Millimeter waves (mm-Waves) can offer large spectrum bandwidths, provide data rates of multi-Gbps and enable seamless connectivity among various devices in the future wireless network (5G and beyond). Incorporating mm-Wave microstrip antennas in $5 \mathrm{G}$ networks will be highly instrumental which provides the advantages of miniaturization, wider bandwidth, narrow radiation pattern, and are better suited for the data rates enhancement with lower delays. In this paper, a low cost, compact size and bandwidth efficient dual-band rectangular microstrip patch antenna is proposed for 5G mm-Wave wireless access and backhaul applications. The antenna has a small size of $7.18 \times 8.38 \times 0.78 \mathrm{~mm}^{3}$. The design has been carried out at $35.786 \mathrm{GHz}$ (Ka band) and $72.709 \mathrm{GHz}$ in the E-band of mm-Wave spectrum, with large bandwidth of 10.435 and $6.705 \mathrm{GHz}$ respectively. The $5 \mathrm{G}$ dual-band antenna has a sufficient realized gain of 8.01 and $8.52 \mathrm{dBi}$ and achieves return loss of -19.833 and $-30.314 \mathrm{~dB}$ at the resonant frequencies. The proposed antenna design can be further extended to be an antenna array of multiple elements to satisfy application requirements and improve the different antenna parameters such as gain, directivity, bandwidth and VSWR.
\end{abstract}

Keywords: Dual-band Microstrip Patch Antenna, Square Slots, 5G mm-Wave, Access and Backhaul. 
ABSTRACTS

\title{
Design of Wireless Local Multimedia Communication Network (WLMmCN) based on Android Application without Internet Connection
}

R. Q. Shaddad ${ }^{1,2}$, F. A. Alqasemi ${ }^{1}$, S. A. Alfaqih ${ }^{1}$, M. F. Alsabahi ${ }^{1}$, A. T. Fara ${ }^{1}$, K. M. Nejad $^{1}$, E. A. Albukhaiti ${ }^{1}$

${ }^{1}$ Faculty of Computing and Information Technology, University of Science and Technology, Sana'a, Yemen

${ }^{1}$ Faculty of Engineering and Information Technology, Taiz University, Taiz, Yemen

rqs2006@gmail.com

\begin{abstract}
This paper presents design of a wireless local multimedia communication network (WLMmCN) in university campus with ubiquitous feature without Internet connection. The WLMmCN is built with Android application that offers voice and text communication to employees and students using WLAN services. The SIP server is an important part of the private branch exchange (PBX) telecoms network used within any university campus. It is a facilitator to make any communication between two or more endusers. The SIP server mainly handles and manages communication sessions. This study finally estimates the performance of the proposed network and application. For audio calling service, the network achieves uplink data rate of $96 \mathrm{kbps}$ and downlink data rate of $158 \mathrm{kbps}$ between two endusers.
\end{abstract}

Keywords: WLAN, Multimedia Communication Network, WLMmCN, Multimedia Android Software, SIP. 


\title{
A Statistical Channel Propagation Analysis for 5G mmWave at $73 \mathrm{GHz}$ in Urban Microcell
}

\author{
Zaid Ahmed Shamsan ${ }^{1,2}$ \\ ${ }^{1}$ College of Engineering, Electrical Eng. Dept., Imam Mohammad Ibn Saud Islamic \\ University, Riyadh, Saudi Arabia \\ ${ }^{2}$ Faculty of Engineering and Information Technology, Taiz University, Taiz, Yemen \\ shamsandieee.org
}

\begin{abstract}
Millimeter-wave (mmWave) is considered a key technology for 5G wireless communication in order to fulfill the increase rapidly the demand of all services that can 5G offer. However, mmWave channel propagation suffers from several obstacles such as weather conditions, rigorous line-of-sight (LOS) requirement, and short range coverage. In this paper, we simulate the characteristics of a spatial channel for $5 \mathrm{G}$ mmWave at $73 \mathrm{GHz}$. A NYUSIM tool has been used to assist in analyze $73 \mathrm{GHz}$ channel characteristics and investigate the effects of different environment conditions including LOS and NLOS. The simulation uses MIMO-OFDM technology through two main integrated models; the free space path loss model (PL) and the statistical spatial channel model (SSCM). Several simulation results illustrated the 3D channel characteristics, power delay profile (PDP), small scale PDP, and root mean square (RMS) delay spread for both LOS and NLOS.
\end{abstract}

Keywords: Millimeter-waves, MIMO-OFDM, RMS Delay Spread, Path Loss Exponent, Power Received, Channel Propagation. 
Advances in Information Security 
ABSTRACTS

\title{
Robot Networks and Their Impact on Cyber Security and Protection from Attacks: A Review
}

\author{
Daniah Anwar Hasan and Linah Faisal Tasji \\ Information System Department, Computer Science and Engineering College, Taibah Univer- \\ sity, Medina, Saudi Arabia \\ tu4160222@taibahu.edu.sa; tu4160220@taibahu.edu.sa
}

\begin{abstract}
As the Internet growth and cyber warfare continue to change, being aware of issues and threats are continually essential for cybersecurity. One of the threats that continue to grow and threaten cybersecurity is botnets. Botnets have acquired the researchers' interest over the past years, a great deal to develop the systems that efficiently and effectively would detect the presence of botnets attacks. Botnets could launch massive commands against com-puter systems connected to the Internet to make financial fraud, intricate spam attacks, and manipulate people through social media. Furthermore, many newer botnets have demonstrated increased levels of sophistication and anonymity as it continues to evolve, making it difficult to withstand them effectively. In this paper, a brief overview of botnets is provided, the importance of cybersecurity, the relationship between cybersecurity and botnets. Additionally, a brief review of how botnets attacks work, botnets architectures, threats represented by botnets, and their impact on cybersecurity is provided. In conclusion, the paper suggests approaches prevent botnet at-tacks and ways and tools that could help organizations and individuals improve their cybersecurity to secure information and improve their security level.
\end{abstract}

Keywords: Cyber, Cyber Crime, Cyber Security, Security, Cyber Attacks, Cyber Threats, Botnets, Internet Robots, Robot Networks, Web Zombies, Bots. 
ABSTRACTS

\title{
An efficient Fog-based Attack Detection Using Ensemble of MOA-WMA for Internet of Medical Things
}

\author{
Shilan S. Hameed ${ }^{1,2}$, Wan Haslina Hassan ${ }^{1}$ and Liza Abdul Latiff ${ }^{3}$ \\ ${ }^{1}$ Malaysia-Japan International Institute of Technology (MJIIT), University Teknologi \\ Malaysia, 54100 Kuala Lumpur, Malaysia \\ ${ }^{2}$ Directorate of Information Technology, Koya University, 44023 Koya, Kurdistan \\ Region-F.R., Iraq \\ ${ }^{3}$ Fakulti Teknologi \& Informatik Razak, University Teknologi Malaysia, 54100 \\ Kuala Lumpur, Malaysia \\ shilansamin@gmail.com; wanhaslina.kl@utm.my; liza.kl@utm.my
}

\begin{abstract}
Smart healthcare applications such as smart fitness, smart watches, and elderly remote monitoring devices have reduced the load on traditional healthcare organizations and led to healthier lifestyles. Nevertheless, these devices are under high risk of zero-day attacks because of their fragile nature and network characteristics. Furthermore, few companies developing these devices take care of security requirements. Attack detection using machine learning techniques such as ensemble learning has been adopted to IoT as a second line of defense. However, most of the proposed approaches are either heavy to implement at Internet of Medical Things (IoMT) devices or are cloud based which lead to delay in the detection of attacks. Also, these detections are centralized which are less compatible with IoT. In this study, an ensemble attack detection method is proposed for the detection of stream data attack at fog layer. The base classifiers are stream and incremental based algorithms, which are compatible with IoMT nature and fog devices. A weighted majority algorithm is followed to obtain best accuracy with reduced latency. The results demonstrated that the proposed model is effective for attack detection at fog layer, while it gives better accuracy, higher detection rate and lower false positive rate with average detection time.
\end{abstract}

Keywords: Fog computing, attack detection, smart health, IoMT. 
ABSTRACTS

\title{
A New DNA Based Encryption Algorithm for Internet of Things
}

\author{
Bassam Al-Shargabi and Mohammed Abbas Fadhil Al-Husainy \\ Faculty of Information Technology, Middle East University, Amman-Jordan \\ bshargabi@meu.edu.com, dralhusainy@gmail.com
}

\begin{abstract}
Nowadays, with the widespread of the Internet of Things (IoT) applications in every aspect of our lives. It's urgent to protect the sensitive data such as images generated by IoT devices transmitted through the wireless network. Furthermore, IoT devices are considered constraint devices regarding limited computation resources such as processing and memory size. Thus, classical encryption methods are not appropriate due to their complex computation resources. Therefore, in this paper, we proposed a new lightweight encryption algorithm based on the DNA sequence to be adequate for IoT device's resources. In the proposed algorithm, we utilized the DNA sequence random nature to generate a strong secret key, which is hard to be broken by attackers. The DNA key is used to encrypt images by two simple and robust substitution and transposition operations where they meet the requirements of IoT computation resources and the protection of the transmitted images. Moreover, the experimental results show outstanding results regarding key size, encryption time, and preparation of distortion compared with other encryption algorithms.
\end{abstract}

Keywords: Internet of Things, Data Encryption, DNA Sequence, Image Encryption, Security, Privacy. 
ABSTRACTS

\title{
Watermarking Techniques for Mobile Application: A Review
}

\author{
Aqilah Abd. Ghani ${ }^{1}$, Syifak Izhar Hisham ${ }^{1}$, Nur Alya Afikah Usop ${ }^{1}$, and Nor Bakiah \\ Abd Warif ${ }^{2}$ \\ ${ }^{1}$ Faculty of Computing, College of Computing and Applied Sciences, University Malaysia \\ Pahang, 26600 Pekan, Pahang. \\ ${ }^{2}$ Faculty of Computer Science \& Information Technology, Universiti Tun Hussien Onn \\ Malaysia, 86400 Parit Raja, Johor. \\ aqilahghani.edu@gmail.com
}

\begin{abstract}
This paper reviewed mobile applications schemes for image watermarking techniques used to secure user media files. Due to the increase in number of smartphones and Internet usage especially in today's life, cybercrime rate seems to be increasing too. Thus, an efficient and suitable watermarking scheme for mobile devices needs to be studied and developed. Digital watermarking is an example of a data hiding technique used by researchers. From the analysis, almost all schemes implement Android Operating System for different techniques and media file types. This paper provides an overview and comparison of various mobile applications for image watermarking techniques.
\end{abstract}

Keywords: Mobile Application, Security, Data Hiding, Watermarking, Review 
ABSTRACTS

\title{
Analysis and Evaluation of Template Based Methods against Geometric Attacks: A Survey
}

\author{
Tanya Koohpayeh Araghi ${ }^{1}$, Ala Abdulsalam Alarood ${ }^{2}$, Sagheb Kohpayeh Araghi $^{3}$ \\ ${ }^{1}$ Advanced Informatics School Universiti Teknologi Malaysia, 54100, Kuala Lumpur, \\ Malaysia \\ ${ }^{2}$ College of Computer Science and Engineering, University of Jeddah, 21959 Jeddah, Saudi \\ Arabia \\ ${ }^{3}$ Energy Institute of Higher Learning, Saveh, Iran \\ tanya.koohpayeh@gmail.com, aasoleman@uj.edu.sa, sagheb.ko@gmail.com
}

\begin{abstract}
Template based methods have been identified as one of the acknowledged methods in image watermarking consisting the influential techniques to prevent geometric attacks. These are added features in the watermarked image by the aim of synchronization recognition without including sensitive information. However, up to now, there is no activity to narrow down each of these methods. This paper categorized the solutions for prevention and detection of geometric attacks based on predefined templates considered in digital images. Due to this classification in the proposed solutions, a state of the art investigation is performed to evaluate and compare the improvements in this area during 2003 to 2020 . The goal is to find the critical factors to make an optimum balance between robustness, imperceptibility, capacity, and to increase security of the watermarking techniques planned in template based methods in order to assist the future researchers to achieve an optimum watermarking algorithm for protection of images against Geometric attacks. The experimental results prove the efficacy of utilizing Pyramidal Just Noticeable Difference (PJND) with high accuracy to increase robustness against more attacks in comparison to the other methods while employing curvelet to hide templates is appropriate to make the capacity of method independent from robustness and imperceptibility.
\end{abstract}

Keywords: Template Based Methods; Spatial and Transform Domains; synchronization Attacks. 
ABSTRACTS

\title{
Survey of File Carving Techniques
}

\author{
Nor Ika Shahirah Ramli, Syifak Izhar Hisham, and Mohd Faizal Abd Razak \\ Faculty of Computing, College of Computing and Applied Sciences, University Malaysia \\ Pahang, 26600 Pekan, Pahang, Malaysia \\ norikashahirah@gmail.com, syifakizhar@ump.edu.my, \\ faizalrazak@ump.edu.my
}

\begin{abstract}
File carving is a method that enables researchers to collect data from unallocated memory space. File carving is a technique used to retrieve data other than data recovery technique and data restoration technique. In general, carving is used to retrieve data with the absence of metadata that point to the content. There are various styles of file carving techniques used for types of files, including PDF, JPEG, XML and etc[5]. This paper focuses mainly on the different types of carving techniques commonly used based on particular circumstances. The key contribution of this paper is the study and analysis of current existing file carving techniques for file carving in order to define their functionality. These techniques are also classified according to the carving method styles used and the fragmentation issues as well as the type of a file system since these techniques rely on the content and the file structure of the particular file to be carved. This paper is divided into two sections, the first section is the traditional carving approach and the second section is the advanced carving approach. This paper will be a guide for researchers searching for carving techniques in order to choose the appropriate carving algorithm and to continue improving it in the future.
\end{abstract}

Keywords: carving, file structure, metadata 
ABSTRACTS

\title{
Affecting Factors in Information Security Policy Compliance: Combine Organisational Factors and User Habits
}

\author{
Angraini ${ }^{1,4}$, Rose Alinda Alias ${ }^{2}$ and Okfalisa ${ }^{3}$ \\ ${ }^{1}$ School of Computing, Faculty Engineering, University Technology Malaysia, 81310 Johor, \\ Malaysia \\ ${ }^{2}$ Department of Information System, Azman Hashim International Business School, Univer- \\ sity Technology Malaysia,81310 Johor Malaysia \\ ${ }^{3}$ Department of Informatics Engineering, Faculty Science and Technology, Universitas Islam \\ Negeri Sultan Syarif Kasim, Pekanbaru, Riau \\ ${ }^{4}$ Department of Information System, Faculty Science and Technology, Universitas Islam \\ Negeri Sultan Syarif Kasim, Pekanbaru, Riau \\ Angraini@uin-suska.ac.id
}

\begin{abstract}
Information security policy compliance is one concern of organisations to improve information security, including universities. Previous research has shown that factors that influence user compliance can come from user behaviour and from within the organisation. This study aims to explore the factors of organisation and user habits that affect user compliance with information security policies. The research model proposed used organisational commitment, organisational culture, reward, and habit variables - this research conducted with a case study of public universities in Indonesia by using an online survey. The results indicated that the factors derived from organisational commitment established a positive influence on the user's respectful behaviour. The insignificant organisational culture and reward affected information security policies. User habits also influenced user behaviour in maintaining information security.
\end{abstract}

Keywords: compliance, information security policy, organisation commitment, organisation culture, habit. 
ABSTRACTS

Mitigation of Data Security Threats in Iraqi Dam Management Systems:

\title{
A Case Study of Fallujah Dam Management System
}

\author{
Hussam J. Ali ${ }^{1}$, Hiba Zuhair ${ }^{2}$, and Talib M. Jawad ${ }^{2}$ \\ ${ }^{1}$ Iraqi Commission for Computers and Informatics, Informatics Institute for \\ Postgraduate Studies, Iraq. \\ ${ }^{2}$ College of Information Engineering, Al-Nahrain University, Baghdad, Iraq \\ hussam.technology2018@gmail.com, hiba.zuhirecoie-nahrain.edu.iq, \\ talibaltalib@yahoo.com
}

\begin{abstract}
Dam management systems have risen as the most salient challenge of egovernment in Iraq that needs IT infrastructure, data integrity, and user privacy protection versus the internet threats that rendering such critical infrastructures useless. This conflict is addressed in this paper by reconciling the reliable dam management system into a multi-tier secure model specifically for Fallujah Dam as a presented case of study. To do so, the design of a relational database, the development of dam management framework along with its functionalities are discussed in this paper. Furthermore, a multi-tier secure model is suggested to integrate with the dam management framework to compile the security properties of data, database, and users' privacy. Expectable findings demonstrate how to develop a successful and satisfactory dam management system which meets all technical, security, and organizational requirements in Iraq.
\end{abstract}

Keywords. Dam management system; internet threats; data security; multi-tier secure model. 
ABSTRACTS

Advances in Information Systems 
ABSTRACTS

\title{
Development and Validation of a Classified Information Assurance Scale for Institutions of Higher Learning
}

\author{
Bello Ahmadu ${ }^{1,2}$, Ab Razak Che Hussin ${ }^{1}$ and Mahadi Bahari ${ }^{1}$ \\ ${ }^{1}$ Information Systems, Azman Hashim International Business School \\ Universiti Teknologi Malaysia, Johor Bahru, Malaysia \\ ${ }^{2}$ NDA Library, Nigerian Defence Academy, Kaduna - Nigeria \\ bahmadu@graduate.utm.my, abrazak@utm.my and ma- \\ hadieutm.my
}

\begin{abstract}
Research in the field of information security is growing fast. However, some niches in the field, especially on the security of classified information in higher education is lacking context-specific scales that researchers can readily use. In this study, we developed and validated a scale for assessing classified information (CI) assurance for institutions of higher learning (IHL). Data were collected from a panel of 10 experts: 4 were seasoned information managers from Nigerian Army University, and 6 were senior academicians selected Universiti Teknologi Malaysia using publications in reputable journals as selection criteria. Each panel member rated the initial item pool and also commented on them. The data thus collected were used in computing the scale's content validity index (CVI), while panel members' comments were employed in improving the items. The result confirmed five dimensions, namely organisational structure, regulatory, human, technological and risk management factors for securing $\mathrm{CI}$ in universities. Based on these four dimensions, a 61-item scale was established that could be used in assessing $\mathrm{CI}$ assurance in universities.
\end{abstract}

Keywords: content validity, Content Validity Index (CVI), classified information, information assurance, institutions of higher learning, university. 
ABSTRACTS

\title{
Sustainable e-Learning Framework: Expert Views
}

\author{
Aidrina binti Mohamed Sofiadin \\ International Islamic University, 50728 Kuala Lumpur, Malaysia \\ aidrina@iium.edu.my
}

\begin{abstract}
The efforts toward sustainable development goals in the educational context are of growing importance even in an e-learning perspective. Sustainability aims to improve the e-learning quality since it supports long-term innovation processes while benefiting society, economy, and the environment. In order to portray its prosperous mission, this paper presented the outline of a Sustainable e-Learning Framework (SeLF). This paper aimed to collect the expert perspectives on this framework. Qualitative data were collected through expert interviews during which the utility and usability of the framework were iteratively evaluated and refined. In order to achieve the framework practicability in different contexts, stakeholders from various universities were invited to participate. The expert perceptions and expectations of sustainable elearning in the context of SeLF were presented in this paper. The experts were asked to reflect on the possible impact of SeLF toward sustainable practices at their own university and personal practice of e-learning sustainability. The findings indicated that SeLF can be used as a guideline for developing sustainable e-learning that supports the continuity of e-learning initiatives.
\end{abstract}

Keywords: Sustainable e-learning, e-learning, the triple bottom line, higher education. 
ABSTRACTS

\title{
Derivation of a Customer Loyalty Factors Based On Customers' Changing Habits in E-Commerce Platform
}

\author{
Mira Afrina $^{1}$, Samsuryadi ${ }^{1}$, Ab Razak Che Hussin ${ }^{2}$ and Suraya Miskon ${ }^{2}$ \\ ${ }^{1}$ Fakultas Ilmu Komputer, Universitas Sriwijaya, Palembang, Indonesia \\ ${ }^{2}$ Information Systems Department, Azman Hashim International Business School (AHIBS), \\ Universiti Teknologi Malaysia (UTM) Skudai, Johor, Malaysia \\ miraafrina81@gmail.com, samsuryadi@unsri.ac.id, abrazakeutm.my, \\ suraya@utm.my
}

\begin{abstract}
The electronic commerce (E-commerce) is a field that involved online enterprises which able to accurately predict the future needs from customers and had influenced on important economic included the social implications. The e-commerce also needed to deal with customer loyalty while dealing with customers' changing consumption habits in brought out for adapting to the new situation while needed to modify their online business activities which because of the changing shopping attitudes. Thus, the online shopping must provide trending and satisfied experienced in order to keep the customers be loyal with e-commerce services. Based on this situation, the customer loyalty is compulsory to be maintaining regarding the fast changing technological evolutions trends which become the reason of customer changing habits. As mostly found based on literature review searching techniques, literature sources still lack in this area where the specific focus are more on data privacy and less focus on the perceived usefulness based on customers changing habits in enhancing the customers loyalty. Then, the guidelines which can deal with customers' changing habits in maintaining the customer loyalty of the e-commerce business are needed. This paper is aims to develop a model which contains the factors for dealing the customers' changing habits on e-commerce while parallel with the electronic loyalty (e-loyalty) in maintaining the customers' loyalty attitudes. Those factors were been figured out by the analysis of literature review method and later be significantly implemented for the enhancement of knowledge regarding a new contribution on customer loyalty field for e-commerce platforms.
\end{abstract}

Keywords: Customer Loyalty, Customer Changing Habits, E-commerce 
ABSTRACTS

\title{
Analysis of Multimedia Elements Criteria using AHP Method
}

\author{
Nadiah Mohamad Sofian, Ahmad Sobri Hashim, and Aliza Sarlan \\ Universiti Teknologi PETRONAS, 32610 Seri Iskandar, Perak, Malaysia \\ nadiahsofianegmail.com, sobri.hashimeutp.edu.my, \\ aliza_sarlan@utp.edu.my
}

\begin{abstract}
In recent years, human-computer interaction principles have dramatically expanded in terms of the design and creation of interactive environments for those affected by autism. Due to the state of autistic children, the innovation in technology designed specifically for children with autism, an effort has been made to consider the added importance of this technology in their everyday lives. The user interface plays an important role in terms of visibility, design and accuracy in the representation of the exact amount of content displayed to the intended users. It is thus crucial to develop a good interface design that helps in the learning process, reduces the complexity of the application and increases the user's satisfaction. This involves investigating the multimedia elements of a good interface, specifically the colour, text and images, in the design of a mobile application interface autistic children. This study aims to analyse the multimedia elements criteria based on the proxy user perspective using the Analytical Hierarchy Process (AHP) method. A total of 38 proxy users who were known as caretakers or parents have been given a questionnaire through an online survey. The questionnaires consist of questions on the multimedia elements used in designing a mobile application for autistic children. It is shown that image and colour were the main requirements for multimedia elements, while image size was the big priority for multimedia elements for the designing user interface of mobile application for children with autism.
\end{abstract}

Keywords: autism; multimedia elements; mobile application; Analytical Hierarchy Process (AHP) 
ABSTRACTS

\title{
The Development of a Criteria-Based Group Formation Systems for Student Group Work
}

\author{
Divya Gopal Mohan and Khairul Shafee Kalid \\ Universiti Teknologi PETRONAS, Seri Iskandar, 32610, Perak, Malaysia \\ divya.gopal_23250@utp.edu.my, khairulshafee_kalid@utp.edu.my
}

\begin{abstract}
The ability of students to work in groups to solve a problem or complete a task is important. However, the existing approach of student group formation tend to result in ineffective and unproductive group work as the group members' skills does not fully complement each other and gives an imbalance impact. Although there are methods in coping this redundancy such as selective appointment method whereas students are grouped based on distinctive skill, personality, and others. However, this traditional method consumes time and energy as it requires manual effort. This paper presents the design of a system that facilitates student group formation by grouping the students based on criteria that fulfils the set of skills needed in a group work. The methodology of this study is two-fold. Firstly, group formation and existing group formation mechanism and tools were investigated. Based on the findings of the investigation, the group formation system was developed based on the agile development approach. The system generates student groups that balanced with diverse skills and personality. The group formation system can assist lecturers to form student groups based on student's skills, knowledge, and personality. A small-scale user acceptance testing was conducted with one of the courses at the university. The results of the testing indicated that the lecturer of the course perceived that the system's mechanism of grouping the student is effective and would like to use it in actual setting. The students perceived that the system is useful, and majority would like to use it in an actual setting.
\end{abstract}

Keywords: Teaching and Learning, Student Groupwork, Education. 
ABSTRACTS

\title{
Trusted Factors of Social Commerce Product Review Video
}

\author{
Humaira Hairudin ${ }^{1}$ Halina Mohamed Dahlan ${ }^{1}$ Ahmad Fadhil Yusof ${ }^{2}$ \\ ${ }^{1}$ Information Systems Department, Azman Hashim International Business School (AHIBS), \\ Universiti Teknologi Malaysia UTM Skudai Johor, Malaysia \\ ${ }^{2}$ School of Computing, Universiti Teknologi Malaysia UTM Skudai Johor, Malaysia \\ humairahairudinegmail.com, halina@utm.my, ahmadfadhileutm.my
}

\begin{abstract}
With the advanced technologies nowadays, many people have stepped up their online shopping culture by watching product review videos before making any purchase decision. Mostly, customers feel convenient of online shopping using social commerce because they can get some information about the products from the consumer experience through product review videos. Product review video is created by consumer in order to share information by using multimedia content to attract customers to watch the video. However, the consumer can share on what they purchase using video reviews but trusted factors of product review videos are still unclear. This study implements systematic literature review approach to review articles from previous studies to identify the factors of product review video. Therefore, this study identifies the trusted factors of product review video that can influence the purchase intention of potential customers, which currently are surveying some products that they intend to purchase later on. For future work, this study can be extended by identifying the effects of trusted factors of product review video on customers. In addition, a model of trusted factors for product review video will be developed.
\end{abstract}

Keywords: product review video, online video sharing, user generated video, trusted factors, trusted content, social commerce. 
ABSTRACTS

\title{
Building Information Modelling Adoption: Systematic Literature Review
}

\author{
Hafiz Muhammad Faisal Shehzad ${ }^{1,2}$, Roliana Binti Ibrahim², Ahmad Fadhil Yusof ${ }^{2}$, \\ Khairul Anwar Mohamed Khaidzir ${ }^{3}$, Omayma Husain Abbas Hassan ${ }^{4}$ and Samah \\ Abdelsalam Abdalla ${ }^{2}$ \\ ${ }^{1}$ School of Computing, University Technology Malaysia, Johor Bahru, Malaysia \\ ${ }^{2}$ Department of Computer Science and IT, University of Sargodha, Sargodha, Pakistan \\ ${ }^{3}$ Department of Architecture, Faculty of Built Environment, University Technology Malaysia \\ ${ }^{4}$ University of Khartoum, Sudan \\ b-anwardutm.my, muhammad.faisalduos.edu.pk, roliana@utm.my, \\ ahmadfadhil@utm.my, omaymahusain@gmail.com, sama7_279@yahoo.com
}

\begin{abstract}
Building Information Modeling (BIM) is the collection of Information and Communication Technologies (ICT), interacting policies and procedures. It supports the construction activities from architectural design to three-dimension (3D) modeling, project scheduling, estimation, and facility management. Despite the many benefits and features of BIM, the proliferation of BIM remains limited and is confronted with adoption issues. Although many existing studies discussed BIM adoption from contextual lenses, discipline-focused, there is still a scarcity of comprehensive overview of technology adoption models and framework in BIM research. Therefore, the purpose of this study is to evaluate the existing status of technology acceptance theories, models used, and find the research challenges. Furthermore, to identify the roles of independent constructs, dependent construct, moderator, and mediators in BIM adoption research. Finally, this SLR will help the researchers for further research in the field of BIM adoption.
\end{abstract}

Keywords: Systematic Literature Review (SLR), Building Information Modeling (BIM), Technology acceptance, BIM Adoption 


\title{
Adoption of Smart cities Models in Developing Countries: focusing in Strategy and design in Sudan
}

\author{
Mohmmed S. Adrees ${ }^{1}$, Abdelrahman E. Karrar ${ }^{2}$ and Waleed I. Osman ${ }^{3}$ \\ ${ }^{1}$ College of Computer Science and Information Technology, Albaha University, Albaha, \\ Saudi Arabia \\ ${ }^{2}$ College of Computer Science and Engineering, Taibah University, Almadina, Saudi Arabia \\ ${ }^{3}$ College of Computer Studies, The National Ribat University, Khartoum, Sudan \\ midrees@bu.edu.sa, akarrar@taibahu.edu.sa, \\ walidibrahimosman@gmail.com
}

\begin{abstract}
The world has seen in recent years face intractable challenges, in particular on third world countries represented in poor infrastructure, slowing economic development, decreased budgets, increased populations, which leads to massive pressure on the different services provided by cities which are becoming limited in their ability to respond to these pressures. It was necessary to take advantage of the concept and experiences of different smart cities in the development of the services provided by the city for its inhabitants to enhance the quality of life. Many factors hinder the adoption of smart city solutions. This research work is an attempt to develop a smart city structural framework in developing economies and presents a framework on how to use the strategy and design to upgrade and optimize the city services, future development plans with strategic planning and interaction design. This research work will be useful for policymakers and practitioners in understanding the development of smart cities and will develop the nation's economy by setting business priorities. Khartoum is selected as a case study as the capital of one of the least developed countries.
\end{abstract}

Keywords: Smart City, Developing Countries, Strategy and Design, Sustainable Development. 


\title{
Factors Affecting Customer Acceptance of Online Shopping Platforms in Malaysia: Conceptual model and Preliminary Results
}

\author{
Nabil Hasan Al-kumaim ${ }^{1}$, Gan Wong Sow ${ }^{1}$, Fathey Mohammed ${ }^{2}$ \\ ${ }^{1}$ Faculty of Technology Management and Technopreneurship, Center of Technopreneurship \\ Development (CTeD), Universiti Teknikal Malaysia Melaka (UTeM) Melaka, Malaysia. \\ ${ }^{2}$ School of Computing, Universiti Utara Malaysia (UUM), 06010 Sintok, Kedah Darul Aman, \\ Malaysia. \\ nhs1426@yahoo.com
}

\begin{abstract}
Many customers today are more reliant on online shopping compared to the traditional shopping because of convenience afforded by online shopping platform. However, customers face many choices when using online shopping platform and need to imagine the product quality since it is online. Moreover, there are many problems of inconsistent service quality through online shopping. Therefore, online sellers need to understand customer preferences in order to survive in the competitive environment of online shopping. This study aims to identify the factors affecting customer acceptance of online shopping platform. Based on Technology Acceptance Model, and relevant studies concerning online platform preferred characteristics, a conceptual model is proposed. Seven independent variables (Website content, website design, perceived enjoyment, perceived usefulness, and perceived ease of use, trust, and customer service quality) are used to construct the model. To develop the variables' measurements, the items were adopted from the related literature. The measurement model was validated by administrating a set of 200 questionnaires using convenience sampling to target online shopper respondents in Malaysia. Smart PLS3 tool was used to analyse the collected data. The result showed significance level of reliability and validity of the measurement model. The developed measurement can be used to examine the factors influencing the customer acceptance of online shopping.
\end{abstract}

Keywords: Online shopping platform, technology acceptance, Malaysia, COVID-19 pandemic. 
ABSTRACTS

\title{
Student Compliance Intention Model for Continued Usage of E-Learning in University
}

\author{
Ken Ditha Tania ${ }^{1,2}$, Norris Syed Abdullah ${ }^{1}$, Norasnita Ahmad ${ }^{1}$ and Samsuryadi \\ Sahmin $^{2}$ \\ ${ }^{1}$ Azman Hashim International Business School, Universiti Teknologi Malaysia, \\ Johor Bahru, Malaysia \\ ${ }^{2}$ Sriwijaya University, Faculty of Computer Science, Palembang, Indonesia \\ kenya.tania@gmail.com, norris@utm.my, norasnita@utm.my, \\ syamsuryadieunsri.ac.id
}

\begin{abstract}
Continued usage of e-learning is important and has been a major challenge. The problem continued usage of e-learning is a lack of student participation, unwillingness to learn, lack of motivation, lack of awareness, behavioral habits and cultural resistance. There are many studies proposed models for continued usage of e-learning. However, the previous models have not conducted research from the point of view of student compliance. If there is no student intention to comply with the rules of using e-learning, then continuing use of e-learning is very unlikely. Compliance with regulation can change culture. Compliance can also be used to ensure continued use in a system, such as the continued usage of an Enterprise System and continued usage of mobile social network service. So, this study proposes student compliance intention model for continued usage of e-learning. The result of this study is the proposed model that will help developer, university, and policy maker to develop e-learning application.
\end{abstract}

Keywords: Continued usage of e-learning, Compliance Intention, Student Intention 
ABSTRACTS

\title{
Digital Information and Communication Overload among Youths in Malaysia: A Preliminary Review
}

\author{
Mohamad Ghozali Hassan ${ }^{1}$, Muslim Diekola Akanmu' ${ }^{1}$ Hussein Mohammed Esmail, \\ Abualrejal $^{2}$, Amal Abdulwahab Hasan Alamrani ${ }^{1}$ \\ ${ }^{1}$ Disaster Management Institute (DMI), School of Technology Management and Logistics, \\ College of Business, Universiti Utara Malaysia, Sintok, Kedah, 06010, Malaysia \\ ${ }^{2}$ Knowledge Science Research Lab, School of Technology Management and Logistics, Col- \\ lege of Business, Universiti Utara Malaysia, Sintok, Kedah, 06010, Malaysia \\ ghozalieuum. edu.my
}

\begin{abstract}
The negative impacts on productivity and concentration of living in an always-busy and plugged-in environment is on the fast rise. The advent of information and communication era has resulted in an over-saturation of information to society, and the new phenomenon is known as 'information overload'. Youths are the most probable affected society members who are considered vulnerable to the negative impact that comes with overload information and communication. The advent of information and communication era has led to information overload, whereby understanding an issue, and making decisions are difficult without distraction. Coping with the presence of many social media sites like Facebook, Twitter, WhatsApp, Blogs, E-mails and LinkedIn which are inevitably necessary for daily communication is becoming a day-to-day challenge. Therefore, the aim of this study is to review previous studies based on the challenges of information and communication overload and to give future empirical studies a basic idea in that context. This study will further consider the 'overload of digital information and communication' among students in Malaysia with the intention to produce an academic-enhanced model to mitigate information and communication overload syndrome. The study aims to produce a more comprehensive and attainable model of managing the 'overload of digital information and communication' that can provide a significant impact on students' academic achievement and attitude. The study has practical relevance for parents and educators to understand the basic understanding of the overload of information and communication among youths in Malaysia which helps to shape a healthier and better generation of Malaysia as stated by the Human Capital Policy.
\end{abstract}

Keywords: Academic-enhanced model, information, communication, youth, Malaysia. 


\title{
ABSTRACTS
}

\section{The Effect of Using Social Networking Sites on Undergraduate Students' Perception and Academic Performance at University of Taiz - Yemen}

\author{
Maged Rfeqallah ${ }^{1}$, Rozilah Kasim ${ }^{1}$ Faisal A.M. Ali ${ }^{2}$ and Yahya Abdul Ghaffar ${ }^{2}$ \\ ${ }^{1}$ Department of Technology Management, Universiti Tun Hussein Onn Malay- \\ sia, Johor Bahru, Malaysia \\ ${ }^{2}$ Department: Data science and information technology Faculty of Administra- \\ tive Sciences, University of Taiz - Yemen \\ faalmh@gmail.com
}

\begin{abstract}
In the academic context, social networking sites (SNSs) have reshaped the way university students connect and communicate with each other, and the way they learn, thus influencing their identities and dimensions. This paper aims to investigate the impacts of SNSs use and student's perceptions on their academic performance at university of Taiz. A survey questionnaire was conducted to a total sample of 357 undergraduate students via personal administration and by online platform to gather the initial information on their use of SNSs and the influence on their academic performance. To analyze the collected data, Statistical Package for Social Sciences (SPSS) software was used. The findings of this study revealed that most of the students use SNSs for socializing activities more than for academic purpose. However, the researcher focused on the study of the correlation between the purposes for which students use SNSs and perceptions of the respondents on academic performance. Thus, overall findings showed that there is a positive correlation between the purpose to which students use SNSs and their perceptions toward academic performance. This means if the purpose of the use of SNS for academic purpose increased, the positive perception of academic performance is increased.
\end{abstract}

Keywords: Social networking sites, undergraduate students, developing countries. 
ABSTRACTS

\title{
Building Information Modelling Adoption Model for Malaysian Architecture, Engineering and Construction Industry
}

\author{
Hafiz Muhammad Faisal Shehzad ${ }^{1,2}$, Roliana Binti Ibrahim ${ }^{1}$, Ahmad Fadhil Yusof ${ }^{1}$, \\ Khairul Anwar Mohamed Khaidzir ${ }^{3}$, Muhammad Mahboob Khurshid ${ }^{4}$ and Farah \\ Zeehan Othman ${ }^{5}$ \\ ${ }^{1}$ School of Computing, University Technology Malaysia, Johor Bahru, Malaysia \\ ${ }^{2}$ Department of Computer Science and IT, University of Sargodha, Sargodha, Pakistan \\ ${ }^{3}$ Department of Architecture, Faculty of Built Environment, University Technology Malaysia \\ ${ }^{4}$ Department of Examinations, Virtual University of Pakistan, Lahore, Pakistan \\ ${ }^{5}$ Bahagian Biasiswa dan Tajaan, Kementerian Pendidikan Malaysia \\ muhammad.faisaleuos.edu.pk
}

\begin{abstract}
Building Information Modeling (BIM) is the collection of Information and Communication Technologies (ICT), interacting policies and procedures. It supports the construction activities from architectural design to three-dimension (3D) modeling, project scheduling, estimation, and facility management. Despite the many benefits and features of BIM, the proliferation of BIM in the Malaysian construction industry is confronted with adoption issues. Therefore this research study intends to find the effect of the factors on BIM adoption in Malaysian Architecture, Engineering and Construction (AEC) and to propose the BIM adoption model. A quantitative approach is adopted with data collection from industry professionals. The proposed model theoretical foundations are based on Technology, Organization Environment framework. The model is tested and validated with the Smart PLS tool. The findings of the study indicate that relative advantage and interoperability are drivers of the BIM adoption. The financial constraints are barriers to BIM adoption. Finally, this study provides recommendations to AEC stakeholders to address issues related to the BIM adoption for enhancing its diffusion in Malaysia.
\end{abstract}

Keywords: Factors; BIM adoption Model; Building Information Modeling (BIM), Technology Acceptance; BIM Adoption 
ABSTRACTS

\title{
Digital Government Competency for Omani Public Sector Managers: A Conceptual Framework
}

\author{
Juma Al-Mahrezi, Nur Azaliah Abu Bakar, and Nilam Nur Amir Sjarif \\ Razak Faculty of Technology and Informatics, Universiti Teknologi Malaysia, \\ Jalan Sultan Yahya Petra, 54100 Kuala Lumpur, MALAYSIA \\ jumaphd2016@gmail.com; azaliah@utm.my; nilamnur@utm.my
}

\begin{abstract}
The government invests in a transformation initiative called Digital Government or previously known as e-Government. Digital Government requires technology, people, and process along with a set of strategies. For the digital government to succeed, government workers must be equipped with appropriate digital skills that are currently not thoroughly explored. Government employees must be trained with proper digital skills, albeit rigorously studied, to ensure the success of this Digital Government implementation. In Oman, studies on digital government started in 2004. However, most of the studies focus on strategy, process, and technology but lack the people aspect. Also, there is a lack of studies on Digital Government Competency (DGC) for public sector managers. Additionally, studies on how to retain employees with advanced ICT competency in different public sector organisations have to be conducted. Therefore, this study aims to develop a conceptual framework for the digital government to assess the relationship between public sector managers, digital leadership skills, data protection skills, soft skills, digital literacy, management skills, digital creativity and innovation. This framework's development relies on Human Capital Theory (HCT) and Technology Organization Environment Theory (TOE). Based on previous literature works, there is a relationship between the success of digital government initiatives and employees' competency. As a result, this study proposes a framework for researchers and governments by demonstrating the value of the Digital Government Competency on the Omani government, which can help to increase the success rate of the Omani Digital Government initiatives.
\end{abstract}

Keywords: Competency, Digital Government, Human Capital Theory, Public Sector, Technology Organization Environment Theory. 
ABSTRACTS

Computational Vision and Robotics 
ABSTRACTS

\title{
Landmark Localization in Occluded faces using deep learning approach
}

\author{
Zieb Rabie Alqahtani ${ }^{1,2,3}$, Mohd Shahrizal Sunar ${ }^{1,2}$, Abdulaziz A. Alashbi ${ }^{1,2}$ \\ ${ }^{1}$ Media and Game Innovation Centre of Excellence, Institute of Human Centered Engineering, \\ Universiti Teknologi Malaysia, 81310 Skudai, Johor, Malaysia \\ ${ }^{2}$ School of Computing, Faculty of Engineering, Universiti Teknologi Malaysia, 81310, Skudai, \\ Johor, Malaysia \\ ${ }^{3}$ Deputyship of Communication and Digital Infrastructure, Ministry of Communication and In- \\ formation Technology, Riyadh, Kingdom of Saudi Arabia \\ asaabdulaziz2@live.utm.my; shahrizal@utm.my; \\ zralqahtani@graduate.utm.my
}

\begin{abstract}
Detecting and localizing facial landmark in occluded faces is a challenging problem for face landmark detection in computer vision. The challenge turns to be more difficult when the occlusion is high where most of the face is veiled. High occluded faces landmark localization is an ongoing research gap which motivates more accurate and highly efficient solutions. This paper presents a review of recent advances in facial landmark detection and localization, discusses available datasets and investigates the influence of occlusion on the accuracy, performance, and robustness on landmark detection. It outlines existing challenges in dealing with and controlling of occlusion.
\end{abstract}

Keywords: Facial Landmark Detection, Face Detection, Computer Vision, Face Recognition; deep neural networks. 


\title{
Contrast Image Quality Assessment Algorithm Based on Probability Density Functions Features
}

\author{
Ismail Taha Ahmed ${ }^{1}$, Chen Soong Der ${ }^{2}$, Norziana Jamil ${ }^{3}$, Baraa Tareq Hammad ${ }^{1}$ \\ ${ }^{1}$ College of Computer Sciences and Information Technology, University of Anbar, Anbar, \\ Iraq. \\ ${ }^{2}$ College of Graduate Studies, Universiti Tenaga Nasional, Malaysia. \\ ${ }^{3}$ College of Computing and Informatics, Universiti Tenaga Nasional, Malaysia \\ ismail.taha@uoanbar.edu.iq; baraa.tareq@uoanbar.edu.iq; Chen- \\ soong@uniten.edu.my; norziana@uniten.edu.my
}

\begin{abstract}
Recently, the existing image quality Assessment algorithms (IQAs) works focusing on distorted images by compression, noise and blurring. Reduced-reference Image Quality Metric for Contrast-changed images (RIQMC) and No Reference-Image Quality Assessment (NR-IQA) for Contrast-Distorted Images (NR-IQA-CDI) have been created for CDI. For each of the five global feature that used in NR-IQA-CDI, The statistical model or the Probability Density Function (PDF) was determined using a Sun2012 database which containing a wide variety of natural scene images. NR-IQACDI showed poor performance in two out of three image databases, where the Pearson Correlation Coefficient (PLCC) were only 0.5739 and 0.7623 in TID2013 and CSIQ database, respectively. For this reason, we present the NR-IQA-CDI based on Monotonic Probability Density Functions (PDFs) (NR-IQA-CDI-MPCF) to address the problem of the existing bell-curve-like PDF of contrast features that cannot reflect the monotonic relation between contrast feature values and perceptual image quality. The findings indicate that the NR-IQA-CDI-MPCF outperforms the current NR-IQA-CDI, especially in the TID2013 database.
\end{abstract}

Keywords: NR-IQA-CDI; Bell-curve; monotonic relation; NR-IQA-CDI based on Monotonic Probability Density Functions (PDFs) (NR-IQA-CDI-MPCF). 
ABSTRACTS

\title{
The Impact of Data Augmentation on Accuracy of COVID-19 Detection Based on X-ray Images.
}

\author{
Yakoop Qasim, Basheer Ahmed, Tawfeek Alhadad, Habeb Al-Sameai and Osamah \\ Ali \\ Department of Mechatronics \& Robotics Engineering, Taiz University, Yemen. \\ yakoopra@gmail.com, balnakar9200@gmail.com, tawfeekhadad@gmail, \\ habeebabdu.alkhaliq1230@gmail.com and osamahfarhan1995@gmail.com
}

\begin{abstract}
COVID-19 is the most common epidemic that attacks the immune system. It was the reason of the death of more than 700 thousand people. A lot of studies made by researchers focused on diagnosing COVID-19 by using deep learning technology. In this paper, we presented a convolutional neural network based on the VGG-16 model architecture to diagnose COVID-19 based on X-Ray images. The Data Augmentation technique was used to increase the number of images related to the COVID19 class from 219 images to 1000 images. After training the proposed model on 2100 images for three classes (COVID-19, Normal and Viral pneumonia) and evaluating its performance on 900 images, we obtained an overall accuracy of $96.3 \%$. This result was higher than the result achieved without using Data Augmentation technique which was 94.4\% and other studies results. We conclude that the Data Augmentation technique is very effective with X-Ray images and it has significantly improved the model performance.
\end{abstract}

Keywords: Deep Learning, Data Augmentation Technique, Convolutional Neural Networks, COVID-19, Transfer Learning. 
ABSTRACTS

\title{
A Fusion Schema of Hand-Crafted Feature and Feature Learning for Kinship Verification
}

\author{
Mohammed Ali Almuashi ${ }^{1}$, Siti Zaiton Mohd Hashim², Nooraini Yusoff², \\ Khairul Nizar Syazwan ${ }^{2}$ \\ ${ }^{1}$ Universiti Teknologi Malaysia, Johor Bahru, Malaysia and Jeddah University, Jeddah, \\ Kingdom of Saudi Arabia \\ ${ }^{2}$ Universiti Malaysia Kelantan, Institute for Artificial Intelligence and Big Data, Pengkalan \\ Chepa, 16100 Kota Bharu, Kelantan, Malaysia \\ mohd.almuashi@gmail.com, sitizaiton@umk.edu.my, \\ nooraini.y@umk.edu.my, nizar.w@umk.edu.my
}

\begin{abstract}
The rapid progress of technology is remarkable and becomes more widespread in various forms such as social networks, smart phones, and high-definition cameras. In this context, analysing facial to kinship based on digital images is a new research topic in computer vision and has been increased dramatically in recent years. In this paper, we trying to detect the relationships between pairs of face images which is reflected a verification matter: given a pairs of face images with a view to find out and infer kin from the non-kin. For this, we proposed a method define by a fusion scheme composed of feature learning (high-level feature) and hand-crafted feature (low-level feature) along with features subtracting absolute value for face pair. For hand-crafted, we apply a histogram of oriented gradients (HOG) descriptor, while, convolutional neural net- works $(\mathrm{CNN})$ is to represent the feature learning. In our experiment to validate the proposed method we apply restricted protocol setting. The proposed method is tested and evaluated on the benchmark databases KinFaceW-I and KinFaceW-II, and the verification accuracies of $68.6 \%$ and $73.5 \%$ were achieved, respectively.
\end{abstract}

Keywords: kinship verification, hand-crafted feature, feature learning, fusion. 
ABSTRACTS

\title{
Lossless Audio Steganographic Method Using Companding Technique
}

\author{
Ansam Osamah Abdulmajeed \\ College of Computer Sciences and Mathematics, University of Mosul, Mosul, Iraq \\ ansam_osamah@uomosul.edu.iq
}

\begin{abstract}
The objective of the work presented here was an implementation of lossless steganographic method on audio files in the frequency domain. The main contribution here was to use of companding technique in audio files to preserve the secret data reversibility with less influence on the signal. This was achieved by making the greatest changes in the less significant coefficients. In this work, secret bits were hidden in the detail components of the first level of integer wavelet transform using companding technique. Location map was created, to prevent samples' overflow/underflow resulting from companding technique, and losslessly compressed using a proposed compression method. Subsequently, it was embedded in the approximation components of that level using LSB replacement. Prior to embedding the compressed location map, the proposed method used Fredkin gate to jumble both of the original LSBs and the compressed location map under controlling of a long secret key. This process was used to increase the security and preserve the reversibility. Results showed that the proposed method kept good quality of the stego-audio (SNR was above $30 \mathrm{~dB}$ ), as well as restored the cover audio without any loss. In addition, the proposed compression method for location map achieved acceptable compression ratio. Furthermore, the security level was increased by use of Fredkin gate. In conclusion, companding technique can be used to achieve lossless data hiding with negligible effect on the audio quality when it is applied on the less significant coefficients.
\end{abstract}

Keywords: Lossless Data Hiding, Steganography, Integer Wavelet Transform, Companding Technique, Fredkin Gate. 
ABSTRACTS

\title{
Smart Traffic Light System Design based on Single Shot MultiBox Detector (SSD) and Anylogic Simulation
}

\author{
E R Salim, A B Pantjawati, D Kuswardhana, A Saripudin, N D Jayanto, \\ Nurhidayatulloh, L A Pratama \\ Department of Electrical Engineering Education, Universitas Pendidikan Indonesia, Jl. Dr. \\ Setiabudhi 207, Bandung 40154, Indonesia \\ arjunibeupi.edu
}

\begin{abstract}
Traffic lights, which can optimize vehicle flow rates, solve congestion, and reduce accidents, are often found on every city road. At some intersections, the traffic light transition duration is still set manually without taking into account the number of vehicles, resulting in longer vehicle queues. This paper proposes a smart traffic light system that can overcome these problems. The system is divided into two main parts, namely detection of objects and determining the duration of the traffic light. In the object detection process, this system uses computer vision technology through the singleshoot multibox detector (SSD) algorithm to detect the number and average speed of passing vehicles. The data then become input for the Anylogic simulation to determine the optimal green light duration for traffic lights. After obtaining the optimal duration, this system will simulate the flow rate of the vehicles. In the trial stage, this system gave good results with the average number of vehicles increasing by around 156 vehicles with an average travel time of 6 seconds faster.
\end{abstract}

Keywords: Anylogic, Single Board Computer, Smart Traffic Light, Singleshoot multibox Detector 
ABSTRACTS

\title{
Learning Scope of Python Coding Using Immersive Virtual Reality
}

\author{
Abdulrazak Yahya Saleh, Goh Suk Chin, Roselind Tei, Mohd Kamal \\ Othman, Fitri Suraya Mohamad \& Chwen Jen Chen \\ ${ }^{1}$ FSKPM Faculty, University Malaysia Sarawak (UNIMAS), Kota Samarahan, \\ 94300 Sarawak, Malaysia \\ ysahabdulrazak@unimas.my
}

\begin{abstract}
Programming is a highly sought-after technical skill in the job market, but there are limited avenues available for training competent and proficient programmers. This research focuses on evaluating an immersive virtual reality (VR) application that has been introduced in the field of Python learning, which uses the interaction technique and a user interface, allowing the novice to engage in VR learning. 30 participants were recruited for the evaluation purpose and they are divided into two groups-15 for Experiment I, and 15 for Experiment II. A questionnaire to evaluate the user interface was done in Experiment I, and a questionnaire to evaluate the novice's acceptance of the VR application was given to the participants in Experiment II. Furthermore, interviews were conducted to collect detailed feedback from all the participants. From the results, it can be noted that the implemented interaction designs in this VR application are adequate. However, more interaction techniques can be integrated to increase the degree of immersive experience of the user in the application. Besides, the interface of the application is considered adequate and reasonable. Nevertheless, there is room for improvement in the aspect of usability and provide a higher level user experience. The novices' acceptance level of the new proposed learning method is low; this might be due to the users' fear of change-- a normal human behaviour in embracing new things in life. Therefore, a larger sample size is proposed to further investigate the novice's acceptance of the new learning method by using an improved version of the VR application.
\end{abstract}

Keywords: Immersive Virtual Reality, Education, Programming Learning. 
ABSTRACTS

\title{
Automatic Audio Replacement of Objectionable Content for Sri Lankan Locale
}

\author{
Gobiga Rajalingam' Janarthan Jeyachandran, M. S. M. Siriwardane ' Tharshvini \\ Pathmaseelan, R.K.N.D. Jayawardhane and N. S. Weerakoon \\ Rajarata University of Sri Lanka,Mihinthale, Sri Lanka. \\ janaroy21@gmail.com
}

\begin{abstract}
Fake news, hate speech, crude language, ethnic and racial slurs and more have been spreading widely every day, yet in Sri Lanka, there is no definite solution to save our society from such profanities. The method we propose detects racist, sexist and cursing objectionable content of Sinhala, Tamil and English languages. To selectively filter out the potentially objectionable audio content, the input audio is first preprocessed, converted into text format, and then such objectionable content is detected with a machine learning filtering mechanism. In order to validate its offensive nature, a preliminary filtering model was created which takes the converted sentences as input and classifies them through a binary classification. When the text is classified as offensive, then secondary filtering is carried out with a separate multi-class text classification model which classifies each word in the sentence into sexist, racist, cursing, and non-offensive categories. The models in preliminary filtering involve the Term Frequency-Inverse Document Frequency (TF-IDF) vectorizer and Support Vector Machine algorithm with varying hyperparameters. As for the multi-class classification model for Sinhala language, the combination of Logistic Regression (LR) and Countvectorizer was used while the Multinomial Naive Bayes and TF-IDF vectorizer model was found suitable for Tamil. For English, LR with Countvectorizer model was chosen to proceed. The system has an $89 \%$ and $77 \%$ accuracy of detection for Sinhala and Tamil respectively. Finally, the detected objectionable content is replaced in the audio with a predetermined audio input.
\end{abstract}

Keywords: Natural Language Processing, Machine Learning, Speech Recognition 
ABSTRACTS

\title{
A Comparison of CNN and Conventional Descriptors for Word Spotting Approach: Application to Handwritten Document Image Retrieval
}

\author{
Ryma Benabdelaziz ${ }^{1}$, Djamel Gaceb ${ }^{1}$, Mohammed Haddad ${ }^{2}$ \\ ${ }^{1}$ LIMOSE Laboratory, University M'Hamed Bougara of Boumerdes, ALGERIA \\ ${ }^{2}$ LIRIS Laboratory, Claude Bernard Lyon 1 University, Villeurbanne, FRANCE \\ \{r.benabdelaziz, d.gaceb\}@univ-boumerdes.dz, \\ mohammed.haddad@univ-lyonl.fr
}

\begin{abstract}
Natural images are easier to represent in feature space than textual images due to the reduced complexity and thus do not require greater learning capacity. The visual information representation is an important step in content-based image retrieval (CBIR) systems, used for searching relevant visual information in large image datasets. The extraction of discriminant features can be carried out using two approaches. Manual (conventional CBIR), based on preselected features (colors, shapes, or textures) and automatic (modern CBIR) based on auto-extracted features using deep learning models. This second approach is more robust to the complexity relative to textual images, which require a deep representation reaching the semantics of text in the image. DIRS (Document Image Retrieval Systems) are CBIR systems related to documents images that propose a set of efficient word-spotting techniques, such as the interest points based techniques, which offer an effective local image representation. This paper presents an overview of existing word retrieval techniques and a comparison of our two proposed wordspotting approaches (interest points and CNN description), applied on handwritten documents. The results obtained on degraded and old Bentham datasets are compared with those of the literature.
\end{abstract}

Keywords: Interest Points, CNN Network, Local and Global Features, Word Retrieval, Word-Spotting, CBIR. 


\title{
Handwritten Arabic Characters Recognition: Comparison of Conventional Machine Learning and Deep Learning Approaches
}

\author{
Faouci Soumia ${ }^{1}$, Gaceb Djamel ${ }^{1}$ and Mohammed Haddad ${ }^{2}$ \\ ${ }^{1}$ Laboratory of Computer Science, Modeling, Optimization, and Electronic Systems \\ (LIMOSE), FS, M'Hamed Bouguerra University of Boumerdès, Algeria \\ ${ }^{2}$ Lab LIRIS, UMR CNRS 5205, University of Claude Bernard Lyon 1, F69622, \\ Villeurbanne, France \\ \{s.faouci,d.gaceb\}@univ-boumerdes.dz, mohammed.haddad@univ- \\ lyonl.fr
}

\begin{abstract}
Over the last decades, automatic handwriting recognition has received a lot of attention, as it is a crucial component for many applications in various fields. Research for this issue has focused on handwriting recognition in Latin languages and fewer studies have been dedicated to the Arabic language. In this paper, we propose and compare two approaches to classifying Arabic characters. The first is based on conventional machine learning using the SVM classifier by comparing different sets of features, most commonly used in the pattern recognition field. The second is based on deep learning by testing different CNN (convolutional neural networks) architectures, which brings a self-characterization of Arabic features. In this context, a new fast and simplified CNN architecture is proposed. We also test different transfer learning strategies on two versions of the OIHACDB dataset and the AIA9K dataset proposed in the literature. In the experimental section, we show that the proposed CNN model achieves accuracies of $94.7 \%, 98.3 \%$, and $95.2 \%$ on the test set of the three databases OIHACDB-28, OIHACDB-40, and AIA9K respectively. Our experiments enrich the tests already carried out on these datasets and show good results in comparison with the literature.
\end{abstract}

Keywords: CNN, Deep learning, Arabic handwritten character recognition, Transfer learning, Feature extractor (FE), Fine-tuning (FT). 
ABSTRACTS

\title{
Document image edge detection based on a local hysteresis thresholding and automatic setting using PSO
}

\author{
Mohamed Benkhettou ${ }^{1[0000-0002-3704-0079]}$, Nibel Nadjeh ${ }^{20000-0001-8570-8654]}$, Djamel Gaceb ${ }^{1}$ \\ ${ }^{1}$ M'hamed Bougara University, LIMOSE Laboratory, Boumerdès, Algeria \\ ${ }^{2}$ Higher National School of Computer Science - ESI, LMCS Laboratory, Algiers, Algeria \\ M.Benkhettou@Outlook.fr, N.Nadjeh.esi@gmail.com, d.gaceb@univ- \\ boumerdes.dz
}

\begin{abstract}
The problem of image segmentation is a persistent problem that fits within the framework of computer vision. We can see year after year new trends bringing in the research's latest advances in the hope of reaching a goal, the one of having the optimal and ideal segmentation of images. In this article, we implement an optimization mechanism using PSO algorithm, it provides the key elements for a good analysis of the impact of parameterization on the quality of segmentation. We propose an improvement of Pratt's metric which aims at providing symmetry, the use of a distance map favourable to computation times, a locally adaptive hysteresis thresholding approach which seems promising, and another one based on Hossain's works. Our experiments have been performed on a new image bank that we have built by merging document image datasets presented during the DIBCO competitions, setting up a multitude of different challenges, varying from images of printed texts to degraded manuscripts.
\end{abstract}

Keywords: Image segmentation, Edge detection, Quality evaluation, Combinatorial optimization, PSO. 
ABSTRACTS

Fast I2SDBSCAN based on integral volume of 3D

histogram: application to color layer separation in document images

\author{
Zakia KEZZOULZA and Djamel GACEB \\ LIMOSE Laboratory, University M'Hamed Bougara of Boumerdes, ALGERIA \\ $\{z$.kezzoula, d.gaceb\}@univ-boumerdes.dz
}

\begin{abstract}
The optical reading of administrative documents using automatic analysis and recognition is a very demanding area in terms of the document quality in order to guarantee effective recognition of their content. Nowadays, when using color in administrative documents, digital experts have seen the importance of color support in facilitating access to the content of scanned documents, especially in the presence of quality degradation, stamps, handwritten notes, and marks on the text. In order to meet this current need, we propose a new method of color layer segmentation intended for document images. This makes it possible to simplify the separation and access to certain information which is very complex or impossible to extract from the image without color processing. It is a new variant of the original DBSCAN approach (called I2SDBSCAN, for integral double space DBSCAN), adapted to pixel clustering of document images guided by color densities. The use of integral volume in 3D color histogram and the coupling between Cartesian and colorimetric spaces have made it possible to considerably reduce the computation times. Experiments prove the effectiveness of the proposed method.
\end{abstract}

Keywords: Color Image Preprocessing, Clustering, Document Image Segmentation, Fast I2SDBSCAN, 3D Color Histogram, Integral Volume. 
ABSTRACTS

\title{
Enhancing daily life skills learning for children with ASD through Augmented Reality
}

\author{
Rahma Bouaziz ${ }^{1,2}$, Maimounah Alhejaili ${ }^{1}$, Raneem Al-Saedi ${ }^{1}$, Abrar Mihdhar $^{1}$ and \\ Jawaher Alsarrani ${ }^{1}$ \\ ${ }^{1}$ Computer Science Department Taibah University, Al Madinah, Saudi Arabia, \\ ${ }^{2}$ ReDCAD, University of Sfax, Tunisia \\ rkammoun@taibahu.edu.sa, h.maimounah@gmail.com, \\ alsaediraneemegmail.com, Ab.rar.mehdar@gmail.com and \\ jawaherahmed.9@gmail.com
}

\begin{abstract}
In addition to traditional therapeutic approaches, several researchers have discussed the use of augmented reality technology over the last decade to promote the development of communication, the learning process, and social behavior for children with Autism Spectrum Disorder (ASD). The proposed work aims to propose a new training system that uses augmented reality to promote the learning of autistic children's daily life skills. This system proposes an interactive environment in teaching children about new skills with related keywords or phrases. We aim to support Autistic Children with fast interaction to learn and repeat the correct behavior.
\end{abstract}

Keywords: Autism Spectrum Disorder (ASD), Augmented Reality (AR), learning, daily life skills. 
Recent Computing and Software Engineering 
ABSTRACTS

\title{
SpaceScience App: Development of a Mobile Applica- tion for School Children
}

\author{
Wan Fatimah Wan Ahmad and Ain Fatihah Ahmad Harnaini \\ Computer \& Information Sciences, Centre of Social Innovation, Universiti Teknologi \\ PETRONAS. 32610 Seri Iskandar, Malaysia. \\ fatimhdeutp. edu.my
}

\begin{abstract}
The inclusion of Science, technology, Engineering and Mathematics (STEM) in the curriculum has been a target for many countries around the world. Nevertheless, the bulk of early STEM education still relies on the conventional teaching model that reaches a subset of students. Most students have opted not to pursue STEM related studies because they are increasingly losing interest in studying STEM. This is due to the limited availability of engaging teaching strategies and resources for more productive learning in the classroom environment. The goal of this project is to research how the application of educational games can help to draw students' interest in the topic of science, develop an educational game application and conduct user experience testing of the educational game application. A mobile application has been developed in the topic of Earth and Space Science for school students aged $10-11$ years old. The application was developed using Ionic Framework, Angular 5, C\#.NET and SQLExpress. An interview and a usability testing have been conducted with schoolteachers and a group of students aged 10 years old. The results showed that most students enjoyed and motivated in learning science by using the mobile application.
\end{abstract}

Keywords: Mobile Application, Science, Technology, Engineering and Mathematics, educational Games 
ABSTRACTS

\title{
Research on Online Problem-Based Learning Among Undergraduate Students: A Systematic Review
}

\author{
Amira Saif and Irfan Umar \\ Centre for Instructional Technology and Multimedia, Universiti Sains Malaysia, Gelugor, \\ Penang, Malaysia \\ Amirsaif77@yahoo.com, irfan@usm.my
}

\begin{abstract}
Problem-based learning (PBL) is a learning method which has been proven to assist student learning. Since its introduction in the 1960's in medical and dental schools in higher learning institutions, this method has been applied in various curriculum settings. The purposes of this review are to identify the most important principles of PBL design as reported in the literature and to identify the aspects that were significantly influenced by online PBL implemented in university context. In this systematic review, several databases were queried using numerous inclusion criteria. A total of 12 studies was extracted and identified from this review. Five PBL principles were identified from this review involving real problem, student-centered, instructor as facilitator, small group size and collaborative work. The finding also indicates that students' perception, critical thinking, knowledge retention and academic achievement were the four aspects frequently studied and significantly influenced by the online PBL method.
\end{abstract}

Keywords: Problem-Based learning, Online Problem-based Learning, PBL principles, Systematic review. 
ABSTRACTS

\title{
Derivation of Factors in Dealing Negative E-WOM for Maintaining Online Reputation
}

\author{
Rizka Dhini Kurnia ${ }^{1}$, Halina Mohamed Dahlan ${ }^{2}$, Samsuryadi $^{1}$ \\ ${ }^{1}$ Fakultas Ilmu Komputer, Universitas Sriwijaya, Palembang, Indonesia \\ 2 Information System Departement, Azman Hashim International Business School (AHIBS), \\ Universiti Teknologi Malaysia (UTM), Skudai, Johor, Malaysia \\ rizkadhini@gmail.com, halinalutm.my, samsuryadi@unsri.ac.id
}

\begin{abstract}
The online reputation is mechanisms which is built in organization and become an essential component in online environment. However, the negative e-WOM becomes threats to the organization. This is happened when the unfavourable messages which been posted on several online platforms negatively influenced other people attitudes and behaviours, and cause negative reputation to the organization. In order to overcome negative e-WOM, the positive e-WOM is significantly needed to deal and maintain the online reputation of the organization. Positive e-WOM offers a greater influence on consumer's perceptions towards the organization. If any organization get a lot of comments as refers to the positive e-WOM through the online conversation among customers, the good online reputation regarding the organization is successfully been achieved. To solve the negative e-WOM towards the organization, the guidelines which can deal with negative e-WOM in dealing and maintaining the online reputation of the organization is needed. The aim of this paper is to develop a model which contains the factors for dealing the threats of online reputation because of the worst spread information by the negative e-WOM. Those factors were derived from the analysis of systematic literature reviews method and later be significantly implement for the enhancement of knowledge regarding a new contribution on online reputation field in organization image and reputation which becomes very important now a days.
\end{abstract}

Keywords: Online Reputation, E-WOM, Negative E-WOM 
ABSTRACTS

\title{
A Terms Interrelationship Approach to Query Expansion Based on Terms Selection
}

\author{
Nuhu Yusuf ${ }^{1,2}$, Mohd Amin Mohd Yunus ${ }^{1}$, Norfaradilla Wahid ${ }^{1}$, Mohd Najib Mohd \\ Saleh $^{1}$, and Aida Mustapha ${ }^{1}$ \\ ${ }^{1}$ Faculty of Computer Science and Information Technology, Universiti Tun Hussein Onn \\ Malaysia, Malaysia \\ ${ }^{2}$ Management and Information Technology Department, Abubakar Tafawa Balewa University \\ Bauchi, Nigeria \\ \{aminy, faradila, najib, aidam\}@uthm.edu.my, ynuhueatbu.edu.ng
}

\begin{abstract}
The term selection is a potential challenge in query expansion. It concerns with the inability of query expansion techniques to select appropriate terms for expanding query. Most of the existing term selection for query expansion techniques allows the expansion of terms with synonyms from a single semantic dictionary, and this doesn't provide an efficient information retrieval system due to low precision results. This paper proposed terms interrelationship of term selection value (TI-TSV) for query expansion where terms are expanded based on synonyms from many semantic dictionaries. The proposed TI-TSV employs existing TSV method of term selection based on the three cases. The three Cases for term selection are based on the retrieved top-ranked documents to increase precision results. The experiments are performing on Yusuf Ali, Sarwar and Arberry English Quran translation datasets collected from Tanzil. The results indicate that the proposed TI-TSV has significantly improved the performance of the search system.
\end{abstract}

Keywords: Term selection, Term selection value, Query expansion, Terms interrelationship, Pseudo-relevance feedbacks, Information retrieval. 


\title{
ABSTRACTS
}

\section{Multi-Domain Business Intelligence Model for Educa- tional Enterprise Resource Planning Systems}

\author{
Hisham Abdullah, Azman Taa and Fathey Mohammed \\ School of Computing, Universiti Utara Malaysia (UUM), 06010 Sintok, Kedah Darul Aman, \\ Malaysia \\ h.alruaini@gmail.com, azman@uum.edu.my, fathey.m.ye@gmail.com
}

\begin{abstract}
The process of applying multi-domains business intelligence for educational Enterprise Resource Planning (ERP) to obtain valuable, analytic, and predictive data still represents a big challenge for educational institutions. This in turn means that the educational institutions, in which the ERP system are implemented, are still lacking analytical, predictive, and reporting functions that improve decision-making. The aim of this research is to design an Educational Multi-Domain Business Intelligence (EduMdBI) model for ERP systems in higher education institutions, which provides better benefits to the students, lecturers, decision-makers, and universities. EduMdBI consists of three major business intelligence domains: Educational Business Intelligence (EduBI) to produce various types of analytic and predictive reports related to academic data, Financial Business Intelligence (FBI) to enable the decision-makers to obtain the required financial analytic and predictive reports, and Performance Business Intelligence (PBI) to produce the required analytic and predictive reports not only related to staff performance but also to educational institution performance. This research concludes that the EduMdBI model can improve the process of obtaining valuable, analytic, and predictive data and help in making the right decision at the right time. Based on the EduMdBI model a Business Intelligence Application can be further designed and developed to include additional sub ERP modules and business intelligence domains.
\end{abstract}

Keywords: Enterprise Resource Planning, multi-domains business intelligence, data warehouse, business intelligence application 
ABSTRACTS

\title{
Measuring Risk Mitigation Techniques in Agile Global Software Development
}

\author{
Adila Firdaus Arbain ${ }^{1}$, Muhammad Akil Rafeek ${ }^{2}$, Zuriyaninatasa Podari ${ }^{2}$ and Cik \\ Feresa Mohd Foozy ${ }^{2}$ \\ ${ }^{1}$ Universiti Teknologi Malaysia, 81310 Skudai, Johor, Malaysia \\ ${ }^{2}$ Universiti Tun Hussein Onn Malaysia, 86400 Parit Raja, Johor, Malaysia \\ adilafirdaus@utm.my, makil399@gmail.com, \\ zuriyaninatasapodari@gmail.com, feresa@uthm.edu.my
}

\begin{abstract}
The main purpose of an Agile development method is to reduce risks, leading to a more successful and effective information system as it had overcome limitations faced in a traditional software development method. This reason lead many to adopt the Agile methodology, especially for global software development (GSD) projects. However, most are not aware of the risks introduced when using agile methods in GSD projects. The risks in GSD projects are dynamic due to factor multiplicity (e.g., multi-locations, multi-groups, multi-cultures, multi-technologies, and multi-standards). This study aims to distinguish the difference between two risk mitigation techniques as an overall technique in tackling the same issue, which is communication issues introduced by the geographical distance between two development teams.
\end{abstract}

Keywords: Agile, Global Software Development (GDSD), Risk Mitigation, Geographical Distance 
ABSTRACTS

\title{
Risk Mitigation Framework for Agile Global Software Development
}

\author{
Zuriyaninatasa Podari ${ }^{1}$, Adila Firdaus Arbain ${ }^{2}$, Noraini Ibrahim $^{1}$ and Endah \\ Surdamilah $^{3}$ \\ ${ }^{1}$ Universiti Tun Hussein Onn Malaysia, 86400 Parit Raja, Johor, Malaysia \\ ${ }^{2}$ Universiti Teknologi Malaysia, 81310 Skudai, Johor, Malaysia \\ ${ }^{3}$ Universitas Muhammadiyah Surakarta, 57162 Jawa Tengah, Indonesia \\ zuriyaninatasapodari@gmail.com, adilafirdaus@utm.my, \\ noraini@uthm.edu.my, Endah.Sudarmilah@ums.ac.id
}

\begin{abstract}
The main goal of Agile development method is to reduce risks so that it leads to a more effective and successful information system. Analyzing priorities, identifying risks are, in fact, important activities in all development approaches, including the Agile development. However, some small studies seek to assess risk management on the basis of Agile global software development (GSD) and to provide mitigation measures to address specific risks. This paper aims to propose a new framework. The mechanism of the proposed framework combines conceptual framework of Global Software Development integrated with risk management in a Scrum framework. The strength of this framework is software development in an Agile process model as a technique to solve identified potential risk in an Agile global environment. As a result, this paper shows the application of Risk Mitigation Framework for Agile Global Software Development (RMAG) in a Scrum process model. Several Scrum practices and phases are used to mitigate risks. We setup an experiment to apply the RMAG in an Agile Global Software Development using Scrum. Each phases have different types of the risk and mitigation technique. Scrum practices help to reduce the risk. Scrum practices and Scrum phases are not provided the outsourcing category because Scrum does not cover the software development life cycle. Thus, this paper can be a reference to developers and Agile practitioners to mitigate or reduce the risk.
\end{abstract}

Keywords: Agile, Global Software Development, Scrum 
ABSTRACTS

\title{
Re-Verification of the Improved Software Project Monitoring Task Model of the Agile Kanban (i-KAM)
}

\author{
Hamzah Alaidaros ${ }^{1,2}$, Mazni Omar ${ }^{2}$, Rohaida Romli ${ }^{2}$, Adnan Hussein ${ }^{1}$ \\ ${ }^{1}$ Faculty of Computer Science and Engineering, Al-Ahgaff University, Mukalla, Hadramaout, \\ Yemen \\ ${ }^{2}$ Human-Centred Computing Research Lab, School of Computing, Universiti Utara Malaysia \\ 06010 UUM Sintok, Kedah, Malaysia \\ m7amza7@yahoo.com,taibh_310@hotmail.com, mazni@uum.edu.my, \\ aida@uum.edu.my
}

\begin{abstract}
The improved software project monitoring task model of the Agile Kanban (i-KAM) is a model that is developed to enhance the progress monitoring task of the software development projects. Although the i-KAM components and criteria have been initially verified through experts' review; however, a re-verification process for the updated version of $\mathrm{i}-\mathrm{KAM}$ is needed after applying the suggestions provided by the experts. Therefore, this paper presents the re-verification results of i-KAM effectiveness, which was assessed based on five factors, which are understandability, relevance, feasibility, organization, and comprehensiveness. In this study, seven software practitioners from different software industries have participated in a focus group to re-verify the revised model. Outcomes show that the revised i-KAM is an effective model characterized by various advantages and numerous features, which can assist software practitioners to effectively monitor their projects' progress during the software development process. Future directions should recognize the practical implementation of iKAM within real environments, focus on the educational and industrial software houses, and validate the model applicability through conducting case studies in developing actual software projects.
\end{abstract}

Keywords: Agile Kanban, Software Development, Software Project, Monitoring Task, Re-verification, Focus Group, i-KAM 


\section{THE $5^{\text {th }}$ INTERNATIONAL CONFERENCE OF RELIABLE INFORMATION AND COMMUNICATION TECHNOLOGY}

Indexing: The books of this series are submitted to ISI Proceedings, SCOPUS, Springerlink, EI-Compendex, DBLP, and Google Scholar 